This item was submitted to Loughborough's Research Repository by the author.

Items in Figshare are protected by copyright, with all rights reserved, unless otherwise indicated.

\title{
Neurophysiology of skin thermal sensations
}

PLEASE CITE THE PUBLISHED VERSION

http://dx.doi.org/10.1002/cphy.c150040

\section{PUBLISHER}

(C) American Physiological Society

VERSION

AM (Accepted Manuscript)

\section{PUBLISHER STATEMENT}

This work is made available according to the conditions of the Creative Commons Attribution-NonCommercialNoDerivatives 4.0 International (CC BY-NC-ND 4.0) licence. Full details of this licence are available at: https://creativecommons.org/licenses/by-nc-nd/4.0/

\section{LICENCE}

CC BY-NC-ND 4.0

\section{REPOSITORY RECORD}

Filingeri, Davide. 2019. "Neurophysiology of Skin Thermal Sensations". figshare. https://hdl.handle.net/2134/23058. 


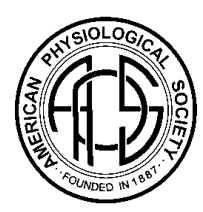

\title{
Comprehensive Physiology
}

Article type: Overview Article

\section{Neurophysiology of skin thermal sensations}

\author{
Davide Filingeri \\ Center for Environmental Design Research, University of California, Berkeley, USA \\ Thermoreception and hygrosensation
}

\begin{abstract}
Undoubtedly, adjusting our thermoregulatory behavior represents the most effective mechanism to maintain thermal homeostasis and ensure survival in the diverse thermal environments that we face on this planet. Remarkably, our thermal behavior is entirely dependent on the ability to detect variations in our internal (i.e. body) and external environment, via sensing changes in skin temperature and wetness. In the past 30 years, we have seen a significant expansion of our understanding of the molecular, neuroanatomical and neurophysiological mechanisms that allow humans to sense temperature and humidity. The discovery of temperature-activated ion channels which gate the generation of action potentials in thermosensitive neurons, along with the characterization of the spino-thalamo-cortical thermosensory pathway, and the development of neural models for the perception of skin wetness, are only some of the recent advances which have provided incredible insights on how biophysical changes in skin temperature and wetness are transduced into those neural signals which constitute the physiological substrate of skin thermal and wetness sensations. Understanding how afferent thermal inputs are integrated and how these contribute to behavioral and autonomic thermoregulatory responses under normal brain function is critical to determine how these mechanisms are disrupted in those neurological conditions which see the concurrent presence of afferent thermosensory abnormalities and efferent thermoregulatory dysfunctions. Furthermore, advancing the knowledge on skin thermal and wetness sensations is crucial to support the development of neuro-prosthetics. In light of the above, this review will focus on the peripheral and central neurophysiological mechanisms underpinning skin thermal and wetness sensations in humans.
\end{abstract}

\section{Introduction}

To everyone who has ever been on the beach in a hot-humid summer afternoon, or out in a cold rainy winter morning, temperature and humidity are common sensations. As familiar as sensing ambient temperature and humidity, sensing the thermal properties of the objects that surround us is also intrinsically part of our existence. The burning hot of walking on fiery sand, the pleasantness of warm gloves, the refreshing coldness of diving into the sea and the icy cold of snow are all thermal sensations which help us defining the characteristics of our surrounding environment. Overall, the ability to sense temperature represents an essential human sensory feature, which is used to initiate or adjust specific behaviors, aiming to maintain homeostasis and ensure survival. 
From an evolutionary standpoint, the ability to sense temperature and humidity has contributed significantly to our success as a species. As homeothermic mammals, humans constantly face the need of autonomically and behaviorally regulate their core body temperature within a narrow range $\left(-36\right.$ to $\left.\sim 40^{\circ} \mathrm{C}\right)$. Living on a planet which is characterized by a variety of thermal environments (e.g. hot and arid deserted landscapes, freezing cold polar regions, tropical and humid areas), we constantly face the challenge of avoiding both body overheating and overcooling, as these can pose a major threat to our survival.

Along with autonomic mechanisms (e.g. regulation of skin blood flow, shivering and sweating), effective body temperature regulation is achieved by consciously seeking for thermally neutral and comfortable environments (e.g. seeking for shade on a sunny day), or by initiating specific behaviors (e.g. donning extra layers of clothing when exposed to cold). Altogether, these behavioral strategies are based upon our ability to detect temperature and humidity variations in our internal (i.e. body) and external environment, via sensing changes in skin temperature and wetness.

As a sensory organ, the skin represents the primary anatomical and physiological interface with our immediate environment. Variations in the thermal properties of the skin (as resulting from changes in body temperature, in the thermal environment and from direct contact with objects) represent the first step in the biological processes which culminate in what we consciously experience as skin thermal sensations. Ultimately, the physiological significance of skin thermal sensations is in driving our thermoregulatory behavior and in contributing to our awareness of the thermal properties of our surroundings.

Although the knowledge on the neural mechanisms underlying skin thermal sensations has grown exponentially over the last 100 years, our understanding of how temperature is peripherally sensed and centrally integrated and of how these physiological processes impact the conscious thermal behavior across the life span, is still far from being conclusive.

Two main reasons drive the interest in advancing the knowledge on the neurophysiology of skin thermal sensations, and both carry clinical implications. Firstly, understanding how thermal and wetness sensations are processed could provide novel insights into the central processing of pain. Temperature sensation has been repeatedly shown to be intertwined with pain processing, both neuroanatomically and neurophysiologically, and in this respect, chronic pain syndrome has been recently proposed as a potential thermosensory deficiency (62). Hence, deepen our understanding of how temperature sensations are processed within the afferent thermo-sensory pathway could be critical in the understanding and treatment of pain. Secondly, advancing the knowledge on the neurophysiology of skin thermal and wetness sensations is crucial to support the development of human-like artificial skin and neuro-prosthetics, which could allow the replication of these unique sensory features in individuals who have experienced limbs' amputation (178). The possibility to restore these sensations in naturalistic contexts through advances in artificial touch could represent a significant breakthrough to improve amputees' quality of life.

Previous Handbook of Physiology articles in Comprehensive Physiology have provided detailed reviews regarding temperature sensation in humans; hoverer, in the 30 years since DarianSmith's latest review on thermal sensibility (76), significant advances have been made regarding the neurophysiology of skin thermal sensations. The discovery of specific molecular candidates for temperature sensation, the characterization of the spino-thalamo-cortical thermosensory pathway, the refinement of neuroimaging techniques which allow mapping of brain regions involved in thermal sensation in awake humans, and the development of neural models for the perception of skin wetness and humidity, are only some of the recent progress which have significantly advanced our understanding of the neurophysiological mechanisms of temperature sensation in humans. 
In light of the above, this article will review the current knowledge on the psychophysical and neurophysiological mechanisms underpinning skin thermal and wetness sensations.

\section{Thermoreception, hygroreception and temperature regulation in humans}

The ability to sense the thermal properties (e.g. temperature and humidity) of the surrounding environment represents one the most important attributes that any animal species, including humans, requires to ensure survival (265). For this reason, species such as humans are provided with specific sensory systems (e.g. amongst which the somatosensory one) which, by transducing distinctive physical properties of external stimuli (e.g. temperature and mechanical pressure) into biological signals (e.g. action potential generated at afferent nerve endings), provide the living organism with awareness (i.e. thermal and wetness sensations) of the characteristics of its surrounding thermal environment (169). Ultimately, the ability to encode sensory information (e.g. thermal and tactile) is used by humans to initiate or adjust specific thermal behaviors (e.g. from adding or removing clothing to migrating to thermally comfortable environments) (262). These behaviors are aimed at maintaining thermal comfort, homeostasis and ensuring survival (241).

As well as contributing to our awareness of our thermal environment and of the thermal properties of the external objects we interact with, skin thermal and wetness sensations play a primary role in autonomic and behavioral temperature regulation. Hence, before reviewing the current knowledge on the psychophysical and neurophysiological bases of human skin thermal and wetness sensations, a brief overview of autonomic and behavioral mechanisms underlying human temperature regulation will be presented.

For a more detailed account of the mechanisms of temperature regulation in man, the reader is referred to the classic work of Hensel (150), to Gagge and Gonzales' Handbook of Physiology article in Comprehensive Physiology (110), as well as to more recent summaries based on evidence from human $(211,253,254)$ and mammalian models $(218,219,225)$

\section{Temperature regulation}

As homeothermic mammals, humans maintain their core (i.e. brain) temperature within a very narrow range $\left(-36\right.$ to $\left.\sim 40^{\circ} \mathrm{C}\right)$ in order to ensure optimal cellular and molecular function $(24,163$, 224). While the significance of maintaining brain temperature at a value $\sim 37^{\circ} \mathrm{C}$ is still under debate in the field of thermoregulation (221), what is well understood is that, due to the variable nature of our surrounding environment, humans constantly face the need of autonomically and behaviorally thermoregulate, as either core overheating and overcooling can pose a major challenge to our survival (241).

The human body prevents core overheating and/or overcooling by achieving thermal balance, a dynamic thermal state which sees a balance between heat gain and heat loss from the body to the environment $(110,277)$. Deep body (core) and skin (shell) temperatures are the principal variables driving the onset of the adaptive responses that regulate the balance between heat production and heat loss from the body to the environment $(25,108,110,212,253,254)$.

The conceptual heat balance equation summarizes the biophysical and environmental factors involved in determining the heat exchanges between the body and the surrounding environment (i.e. thermal audit) (241):

$\mathrm{M}-\mathrm{W}=\mathrm{E}+\mathrm{R}+\mathrm{C}+\mathrm{K}+\mathrm{S}$

Where: 
$\mathrm{M}=$ rate of metabolic energy production $\left(\mathrm{W} \cdot \mathrm{m}^{-2}\right)$

$\mathrm{W}=$ rate of mechanical work $\left(\mathrm{W} \cdot \mathrm{m}^{-2}\right)$

$E=$ rate of evaporative heat loss $\left(\mathrm{W}^{-2}\right)$

$\mathrm{R}=$ rate of radiative heat loss $\left(\mathrm{W} \cdot \mathrm{m}^{-2}\right)$

$\mathrm{C}=$ rate of convective heat loss $\left(\mathrm{W}^{-2}\right)$

$\mathrm{K}=$ rate of conductive heat loss $\left(\mathrm{W} \cdot \mathrm{m}^{-2}\right)$

$\mathrm{S}=$ rate of heat storage $\left(\mathrm{W} \cdot \mathrm{m}^{-2}\right)$

The body metabolic energy production $(\mathrm{M})$ provides energy to perform mechanical work $(\mathrm{W})$ and the net difference between the two $(\mathrm{M}-\mathrm{W})$ represents the amount of energy released by the body as heat. This value is always positive and represents the body heat production. To achieve thermal balance (i.e. $S=0$ ) the heat produced by the body has to be balanced by the heat released to the environment. This occurs via four main physical avenues: evaporation (E), radiation $(R)$, convention $(C)$ and conduction $(K)$. Therefore, for heat balance $(S=0)$ :

\section{$M-W-E-R-C-K=0$}

From a biophysical standpoint, if the value resulting from the above equation is positive, gains in body heat content occur; if negative, body heat loss occur. From a physiological point of view, if heat gains surpass heat losses, body core temperature will rise whereas if the contrary occurs, body core temperature will drop (110). Physical factors such as air temperature, radiant temperature, relative humidity and air velocity significantly contribute to determine these processes.

In humans, thermal balance between heat production and heat loss is achieved by means of autonomic and behavioral thermoregulatory responses.

\section{Autonomic temperature regulation}

Autonomic thermoregulatory responses in humans are triggered by thermal stimulation of various areas of the central nervous system (e.g. medulla oblongata, pons and midbrain). Amongst these, the pre-optic area of the hypothalamus is considered as the main thermal-controller $(150,254)$. By receiving afferent inputs from thermally-sensitive neurons (i.e. thermoreceptors) located peripherally (i.e. skin and muscles) as well as centrally (i.e. brain, spinal cord and viscera) in our body $(24,225,253,261)$, this area provides commands to peripheral thermo-effectors in order to initiate autonomic responses defending body temperature from environmental challenges (163, $224,268)$.

It is now recognized that the thermoregulatory system controls body temperature as a results of the activity in a number of relatively independent effector loops $(218,219,255)$, without the need of a specific "set point" (25). According to the type of external stimuli (i.e. warm or cold) which trigger the activation of peripheral and/or central thermoreceptors, specific autonomic responses are activated. These consist primarily of changes in vasomotor tone (i.e. vasoconstriction and vasodilation) $(164,307)$ and in sudomotor activity (i.e. sweating) $(56,96,211,286)$ as well as in the activation of shivering and non-shivering thermogenesis $(220,225,276,311)$. In case of rises in body temperature (i.e. condition of heat gain), heat losses to the environment are initially facilitated by means of skin vasodilation and subsequently by sweating. In case of drops in body 
temperature (i.e. condition of heat loss), heat losses are initially limited by means of skin vasoconstriction and heat gains are facilitated by means of shivering thermogenesis (110).

The autonomic mechanisms controlled by the pre-optic area of the hypothalamus act as regulators of heat production and heat loss within the body and from the body to the environment. The balance between these processes results in the output core temperature, which in case of resting humans, fluctuates around $\sim 37^{\circ} \mathrm{C}$. Regulation of heat production and heat loss mechanisms is neurally driven by changes in the afferent activity of thermoreceptors located in deep body (e.g. central nervous system and viscera) as well as in more superficial (e.g. skin and muscle) tissues $(25,26,122,144,211,217,218,267)$.

Although powerful, the functional capacity of human autonomic thermoregulation is however limited by physiological and biophysical constraints (262). Maximal sweating as well as maximal vasodilation and vasoconstriction are limited by physiological (e.g. sweat gland density and output, number of capillaries) $(164,266)$ and biological factors (e.g. age) $(36,172,270)$. From a biophysical point of view, anthropometrical characteristics also play a role in limiting the functional ability of the autonomic thermoregulatory system. For example, body surface area to mass ratio is an important parameter for heat exchange, which can limit the ability to dissipate heat to the environment $(69,70,113,140)$. Heat losses are indeed proportional to the gradient between the skin and environment and to the surface area available for heat exchange $(110,112,113,140)$. Thus, given the same body mass, individuals with smaller body surface areas require greater increases in e.g. skin vasodilation and/or sweating than individuals with larger body surface areas, in order to dissipate the same amount of heat to the environment and to prevent core overheating $(69,70,139)$.

Despite these intrinsic physiological limits, humans successfully maintain their thermal balance while being exposed to various extreme environments (e.g. from the moon surface to the Sahara desert), in which autonomic responses alone could not guarantee survival (254). In this respect, what assures survival to our species is the virtually unlimited power of behavioral thermoregulation.

\section{Behavioral temperature regulation}

Behavioral thermoregulation can be defined as any conscious decision taken with the aim of maintaining thermal balance and it represents an infinite resource for human body temperature regulation $(35,106,259,262)$. From simply looking for shade on a sunny hot day (241), to adding or removing clothing (141), humans constantly adjust their thermal behavior in order to maintain thermal comfort $(106,180)$.

As a conscious indicator of thermal balance, thermal comfort is defined as that condition of mind which expresses satisfaction with the surrounding thermal environment, and it is currently considered as the result of the interaction between physical, physiological and psychological factors $(48,111,156,233,295)$. The physical factors refer to the characteristics of the environment to which individuals are exposed (e.g. ambient temperature and humidity) (241). The physiological factors refer to the autonomic thermoregulatory processes used by the human body to maintain thermal homeostasis (110). The psychological factors refer to individual sensations and to the hedonic component of the stimulus (perception) (83). In this context, thermal sensation, affective judgments (i.e. how a person would like to feel) and personal experiences, play a fundamental role in defining thermal preference $(32-34,156)$. The combination of such complex and dynamic psychophysiological factors produces continuous variations in individuals' satisfaction with their thermal environments, and therefore a variety of personal judgments about what is/is not perceived as thermally comfortable $(6,32,33)$. 
From a neuroanatomical point of view, a number of regions of the central nervous system have been identified which contribute to the central integration and processing of sensory information that are then used by humans to actively and consciously adjust their thermal behavior $(106,229$, $251,262)$. Interestingly, as to underline the integrative nature of human autonomic and behavioral thermoregulatory responses, some of these regions (e.g. insular cortex) (62) share behavioral as well as autonomic thermoregulatory functions (92).

It appears clear at this point that the essential physiological feature required to successfully adjust the thermal behavior is the ability to sense the thermal properties of the surrounding environment (272) as well as of one own' s body (68), i.e. the ability to experience thermal and wetness sensations.

\section{Thermoreception and hygroreception}

The ability to sense temperature (i.e. thermoreception) represents an important drive of autonomic and behavioral thermoregulatory responses both in humans and in other mammalian and non-mammalian species $(114,272)$. Detecting the thermal properties of the surrounding environment as well as of one own' $s$ body is made possible by the presence of thermallysensitive neurons (i.e. thermoreceptors) which are found peripherally (i.e. skin and muscles) as well as centrally (i.e. brain, spinal cord and viscera) in the body $(24,225,261)$. Whether located in the skin, muscles, viscera or brain, by responding to thermal changes occurring in their receptive fields, these sensory neurons: a) provide afferent information regarding the thermal properties of the environment and/or of an object with which our skin is in contact (i.e. thermal sensation) (261); b) modulate autonomic thermal responses (e.g. suppression/increases in sweating due to thermal changes in skin or gastro-intestinal temperatures) $(217,222)$.

The anatomical distribution of thermally sensitive neurons, which sees warm sensitive thermoreceptors being present in larger numbers centrally, while cold sensitive thermoreceptors are largely distributed in the periphery (254), highlights the asymmetrical nature of our autonomic thermal physiology. Indeed, the normal core temperature $\left(\sim 37^{\circ} \mathrm{C}\right)$ is closer to its upper $\left(\geq 40.5^{\circ} \mathrm{C}\right)$ than its lower survival limit $\left(\leq 18-20^{\circ} \mathrm{C}\right)(241)$, indicating that rises in core temperature are potentially more dangerous than equivalent drops in this physiological parameter (254).

While thermoreception is without doubt a key human sensory feature, sensing temperature is not the only somatosensory mechanism to contribute to autonomic and behavioral thermoregulatory responses in humans. The ability to sense skin wetness and humidity (i.e. hygroreception) plays indeed an important role in modulating behavioral and autonomic thermal adaptations. Despite its importance in the context of behavioral and physiological function (see Psychophysics and Neurophysiology of skin wetness perception sections), the neurophysiological bases of human skin wetness perception have however only recently started to be unveiled.

Due to their importance in providing the sensory bases for conscious skin thermal sensations and wetness perceptions, and in light of the topic of this review (i.e. neurophysiology of skin thermal sensations), the analysis of the mechanisms of temperature sensation and wetness perception will focus on skin-related sensations. The following overview will proceed by first examining the psychophysical relation between temperature and wetness stimulation of the skin and the resulting thermal and wetness perceptions. Subsequently, a detailed description of the neurophysiological mechanisms underpinning the peripheral integration and central processing of thermal and wetness sensations in humans will be provided. 


\section{Psychophysics of temperature sensation}

Whether resulting from changes in body or ambient temperatures, or from the contact with an external object, thermal stimulation of the skin generates variations in skin temperature which induce non-painful and painful thermal sensations $(128,261)$.

Taking into account the regional variability produced by differences in skin blood perfusion (164), mean skin temperature for a resting standard-sized individual (i.e. body mass $70 \mathrm{Kg}$; body surface area $\left.1.8 \mathrm{~m}^{2}\right)(22)$, exposed to a neutral environment (e.g. ambient temperature $\sim 2{ }^{\circ} \mathrm{C}$ ), ranges from $\sim 30$ to $\sim 34{ }^{\circ} \mathrm{C}(110)$. Starting from this range, cooling or warming of the skin within a temperature range of $\sim 15$ to $\sim 42^{\circ} \mathrm{C}$ generates non-painfully cool or warm thermal sensations ( 76 , 206). Remarkably, skin temperature changes as small as of $0.003^{\circ} \mathrm{C}$ (produced by radiative heating at a rate of $0.001^{\circ} \mathrm{C} / \mathrm{s}$ ) have been shown to trigger thermal sensations (137). Outside the non-painful range, further cooling $\left(<15^{\circ} \mathrm{C}\right)$ or warming $\left(>42{ }^{\circ} \mathrm{C}\right)$ of the skin generates painfully cold or hot thermal sensations $(128,289)$.

Within the non-painful range, subjective thermal sensations are strongly influenced by and can vary largely according to 3 main parameters: 1 ) the initial skin temperature from which the resulting change occurs; 2 ) the rate at which this change occurs; 3$)$ the skin area stimulated (76, $137,138,175-177,234,235)$. Specifically, the psychophysical correlates of skin temperature sensation in man (Et) can be expressed as a function of skin temperature $(T)$, the rate of change of skin temperature over time $(\Delta T / \Delta t)$ and of the stimulus area $(A)$ as follow (151):

$E t \rightarrow f[T,(\Delta T / \Delta t), A]$

Along with these fundamental parameters, the location of the thermal stimulation on the body can also influence subjective thermal sensations (30). Indeed, regional differences in thermal sensitivity across the human body have long been observed (137).

In light of the above, the impact that each one of the four main parameters outlined above exerts on the psychophysics of temperature sensation will be discussed below.

\section{Role of absolute skin temperature}

With regards to the role that absolute skin temperature plays on the way a temperature stimulus is perceived, we know that when the skin is adapted to temperature values ranging from $\sim 30$ to $\sim 34{ }^{\circ} \mathrm{C}$, neither warm nor cool sensations are experienced $(151,176)$. Any linear increase or decrease in skin temperature from this adapting value results in corresponding warm or cool sensations (175), with a temperature threshold (i.e. the relative change in skin temperature required to induce a thermal sensation) which varies according to the rate of temperature change (e.g. the faster the change, the smaller the temperature threshold). However, when skin temperature is adapted to temperatures outside (i.e. above or below) the neutrality range, the temperature threshold for warm and cool sensations is significantly shifted in a particular way: the more the new adapting temperature deviates from the neutrality value (i.e. above or below $\sim 30$ to $\sim 34^{\circ} \mathrm{C}$ ), the greater the change in skin temperature required to induce a thermal sensation (151).

The reason for this increase in temperature thresholds when baseline skin temperature is adapted to values outside the neutrality range is related to the resulting change in the baseline thermal sensation experienced at the new adapting skin temperature. When skin temperature is adapted to values below $\sim 30^{\circ} \mathrm{C}$ or above $\sim 34^{\circ} \mathrm{C}$, persisting cool and warm sensations are respectively experienced, for a duration which is longer the more the new adapting temperature deviates for the neutrality range (177). For example, up to $20 \mathrm{~min}$ are required for $\mathrm{a} \pm 4^{\circ} \mathrm{C}$ change in initial skin temperature to adapt and no longer be perceived as persistently warm or cool (177). When skin temperature is changed to values outside the neutrality range, and full 
adaptation (i.e. absence of warm or cool sensations) has not yet been achieved, any change in skin temperature from these new adapting values will initially result in increases or decreases in the intensity of the persisting sensation (176). For example, if initial skin temperature is adapted to a lower temperature than $-30^{\circ} \mathrm{C}$, a persisting cool sensation is experienced; any increase in temperature from this point will initially result in a reduction in the intensity of the cool sensation (i.e. a less cool sensation is experienced), before a warm sensation is induced $(176,177)$. Figure $1 \mathrm{~A}$ shows how absolute temperature thresholds (i.e. the relative change in skin temperature required to induce a thermal sensation; solid squares, solid curves) and just noticeable difference thresholds (open squares, broken curves) change according to different adapting skin temperatures. Overall, this initial reduction in the intensity of the persisting sensation seems to contribute to an increase in the temperature threshold for detecting changes in skin temperature, when the latter is adapted to values outside the neutrality range.

\section{Role of rate of change in skin temperature}

With regards to the way the rate of temperature change influences the way a temperature stimulus is perceived, we know that the faster the increase or decrease in skin temperature from an adapting temperature of $34^{\circ} \mathrm{C}$, the smaller the change in skin temperature that is required to induce a thermal sensation. On the contrary, the slower the rate of change, the greater the change in skin temperature that is required to induce a thermal sensation $(151,175)$.

For example, Kenshalo et al. (175) showed that when baseline skin temperature $\left(31.5^{\circ} \mathrm{C}\right)$ of the dorsal surface of the forearm of 3 participants was increased/decreased with rates of change of $0.3,0.1,0.05,0.02$, and $0.01{ }^{\circ} \mathrm{C} / \mathrm{s}$, with a thermal stimulator (surface: $14.4 \mathrm{~cm}^{2}$ ), the thermal threshold for both cool and warm sensations varied significantly according to the rate of change: the slower the rate of changes the higher the thermal threshold (Fig. 1B). This effect was particularly evident for rates of temperature change lower than $0.1{ }^{\circ} \mathrm{C} / \mathrm{s}$ and more pronounced on the warm than on the cool side of the thermal sensation spectrum. The more pronounced effect on the warm side seems to be due to the fact that adaptation to temperature changes occurs more rapidly during skin warming than during skin cooling (177). Hence, the faster rate of adaptation to warming, in combination with a slower rate of change in temperature, seems to result in increasing the thermal threshold for skin warming to a larger extent than for skin cooling (175).

Apart from detecting changes in skin temperature from an adapting value, psychophysical studies have provided evidence on the mechanisms underlying the ability to estimate the magnitude of a sudden change in skin temperature (a phenomenon which is representative of what happens in real life when the skin is suddenly exposed to sources of heating or cooling, or when it comes in contact with external objects) $(14,76,130)$. For example, during the contact with a surface with a temperature different form that of the skin, a sudden change in skin temperature occurs as a result of the heat exchange between the skin and the surface touched. The direction and magnitude of this temperature change is driven by the biophysical properties (e.g. heat capacity) of both the skin and the surface touched (14). Interestingly, the temperature change resulting from sudden exposures to sources of heating or cooling often generates supra-threshold changes in skin temperature, whose amplitude has been shown to linearly correlate with the magnitude of the resulting thermal sensations (Fig. 1C) (76).

\section{Role of stimulus area}

With regards to the way the stimulus area influences the way a temperature stimulus is perceived, we know that an increase in the area of the skin exposed to warming/cooling results in a decrease in the temperature threshold required to induce a thermal sensation $(76,151)$. 
Early work from Hardy and Hoppel (137) showed that, when the forehead of human participants was exposed to radiative warming, the temperature threshold for warm sensation decreased significantly when the surface area exposed to the stimulus was increased (i.e. from 0.2 to 40 $\mathrm{cm}^{2}$ ). Similarly, in a study in which radiant and conductive heating were used to test warm temperature thresholds for the forehead, forearm and back, Kenshalo et al. (173) found that increasing the size of the area exposed to warming (within a range of 1 to $14.4 \mathrm{~cm}^{2}$ ), significantly decreased warm temperature thresholds for all the regions investigated, and according to a hyperbolic function. This function is expressed in the following form:

$\mathrm{I}=\mathrm{k} \cdot \mathrm{A}^{-\mathrm{b}}+\mathrm{C}$

where $\mathrm{I}$ is the temperature increment detected by the participant, $\mathrm{k}$ is a constant which changes according to the skin region stimulated, $\mathrm{A}$ is the area of the skin stimulated, $\mathrm{c}$ is the warm temperature threshold when the area of the skin A is very large (e.g. $200 \mathrm{~cm}^{2}$ ), and b is the degree of spatial summation $(76,173)$. According to this function (173), it can be extrapolated that, when a complete spatial summation capable of inducing a thermal sensation occurs (i.e. $b=1$ ), assuming $c$ being very small (due to the large area of skin $A$ ), the skin temperature increments detected by a participant are inversely proportional to the size of the area stimulated. As a result, the same temperature threshold can be achieved by either reducing the area exposed to a stimulus while increasing the skin temperature change, or vice versa. Overall, such observations indicated that spatial summation in temperature sensation occurs and that this can significantly influence the magnitude of sensory responses upon thermal stimulation of the skin.

The concept of spatial summation in the warmth sense was further observed by Stevens et al. (274) in a series of experiments where different regions of the body (i.e. cheek, forearm, calf and back) were exposed to different thermal stimuli (i.e. radiative heating), resulting from combining different irradiance levels (range $=20-500 \mathrm{~mW} / \mathrm{cm}^{2}$ ) and surface areas $\left(\right.$ range $=3.7-200 \mathrm{~cm}^{2}$ ). Interestingly, when the irradiance level was kept constant, participants reported warmer thermal sensations as the stimulus areas was increased. Furthermore, it was found that, while the relationship between stimulus area and irradiance level was similar in shape for all the skin regions investigated, the same regions differed in terms of their levels of sensitivity (e.g. forearm, back and calf were shown to be less sensitive to warmth than the check). Finally, the degree of spatial summation was shown to be greater at lower levels of irradiance. At higher levels of irradiance (i.e. resulting in variations in skin temperature closer to the thermal threshold for heat pain), spatial summation was indeed shown to be significantly reduced, if not absent (Fig. 2). As well as observing an upper temperature threshold, Stevens et al. (274) also found a lower threshold for spatial summation. As part of the same investigation, it was observed that spatial summation did not occur when irradiance levels were below $-3 \mathrm{~mW} / \mathrm{cm}^{2}$, irrespectively of increases in stimulus area (e.g. from 60 to $200 \mathrm{~cm}^{2}$ ). It was therefore suggested that spatial summation could be limited by the amount of thermal stimulation needed to overcome the excitation threshold of skin thermoreceptors which is required to trigger a detectable thermal sensation (274). Altogether, these results indicated that interactions between stimulus area and degree of change in temperature significantly modulate thermal sensations, according to a mechanism of spatial summation $(76,137,173)$, which is however limited by lower (i.e. smallest change in skin temperature required to trigger a thermal sensation) and higher (i.e. threshold for heat pain) thermal thresholds (274). Apart from occurring on hairy regions of the body, spatial summation of thermal stimulation appears to occur also on glabrous skin (e.g. palm of the hand), and particularly when warm stimuli are used. In this respect, the degree of spatial summation in the warmth sense appears to be similar to the one observed to occur when hairy skin is stimulated (84).

Within the context of how thermal information are spatially integrated, an interesting perceptual mechanisms that deserves mention is the one that goes under the name of thermal referral (123, 155). The phenomenon of thermal referral shares phenomenological similarities with visual fillingin, in which the discontinuity in the visual signals of interest can be compensated perceptually on the basis of the spatially adjacent context (245). 
Thermal referral has been recently demonstrated by Ho et al. (155) in a study in which individuals were asked to touch three thermal stimulators simultaneously with the middle three fingers of one hand, and to report thermal sensations for each one of their fingers. As a result of this task, and despite only the outer two stimulators were either cooled or heated, the central (neutral) stimulator was also perceived to be cool or warm, indicating that the adjacent thermal stimulation of the two outer fingers biased thermal sensations related to the central finger. Interestingly, despite the similarities between visual and thermal referral, the latter appears to occur only when concurrent thermal and tactile stimulation of the skin site exposed to the thermal stimuli is present. For example, Green et al. (123) has previously reported that, during a thermal referral task similar to the one described above, the illusory thermal sensation perceived at the central finger disappears if the finger is held (spatially) above the central (neutral) stimulator (123). This observations highlight the importance of cross-modal interactions between thermal and tactile sensing in contributing to the ability to spatially discriminate thermal sensations across the body. Further supporting this view is the fact that the interaction between concurrently applied thermal and tactile stimuli has been repeatedly shown to modulate the way thermal sensations are experienced. For example, Green et al. (125-127) reported an attenuation in the cold sensation evoked by the same mild cooling stimulus (resulting in a $\sim 2^{\circ} \mathrm{C}$ drop in skin temperature) when this resulted from the dynamic application of an already cold thermal probe to the skin, as opposed to when the same thermal probe was sitting on the skin and its temperature was then suddenly changed. (125-127). In line with this, the modulatory role of tactile inputs on thermal sensations has been recently shown in one of this author's investigations, in which individuals reported significantly attenuated cold sensations when the same cold stimuli (i.e. temperature range: 4 to $15^{\circ} \mathrm{C}$ lower than local skin temperature; area of stimulation: $25 \mathrm{~cm}^{2}$ ) were applied with a higher $(10 \mathrm{KPa})$ than with a lower $(7 \mathrm{KPa})$ static pressure. This, despite both high and low pressure stimuli induced similar drops in skin temperature (103).

Altogether, these observations highlight the importance that concurrent tactile stimulation (e.g. as resulting from the contact with an object) plays in contributing to spatial acuity and in modulating thermal sensations $(123,154,155)$. This inter-sensory interaction between thermal and tactile sensing is not entirely surprising, if considering that the thermal sense appears to be lacking of spatial acuity, particularly when thermal stimulation results from non-contact stimuli (e.g. radiant heating) $(38,154)$. Furthermore, these thermal-tactile interactions are in line with the previously described key role that tactile stimuli play in modulating other somatosensory sensations. Amongst these, pain represents a well-known example of how the interaction with tactile stimuli (e.g. in the form of pressure applied on the skin) can significantly reduce the magnitude of this sensation $(23,124-126)$.

\section{Role of skin region stimulated}

With regards to the way the skin region stimulated affects the way a temperature stimulus is perceived, we have long known that regional variations in temperature sensitivity exits across the body, and that these share both perceptual as well as physiological significance (151). Before reviewing the psychophysical evidence on the regional variations in thermal sensitivity, it appears necessary to provide the reader with a brief overview of the concept of cutaneous cold and warm spots, as firstly introduced by Blix (20), Goldscheider (121) and Donaldson (87) [for an historical review of the contribution of these authors to the study of the temperature sense see Norrsell et al. (230)].

In his investigation of cutaneous sensations and of how these could be generated by nerve activity (20), Blix electrically stimulated the skin with electrodes with a thin metal pin and found that when specific spots in the skin where stimulated, cool or warm sensations could be evoked in awake individuals. Blix went further investigating temperature sensitivity of the skin with an apparatus he constructed for temperature stimulation (consisting of two water containers connected to $Y$ shaped tube with a metal tip whose temperature could be controlled by allowing 
water at different temperatures contained in the two containers to flow through the tube) (230). In his temperature stimulation of the skin, Blix found that: 1 ) cold and warm sensations could be evoked from different spots; 2) applying cold stimuli to warm spots rarely induced cold sensations (and vice versa); 3) few spots presented both cold and warm sensitivity; 4) cold spots were in higher number than warm spots. As a result of these experiments, Blix produced body maps of cold and warm spots across the skin (230). Similar results were almost simultaneously obtained by Donaldson (87), who also mapped cold and warm spots across the skin.

These early investigations introduced the concept of skin spots for cold and warm detection and opened to the investigation of whether the distribution of these spots (a potential indicator of regional differences in thermal sensitivity), would be different across the skin (151). As a result, initial studies on the regional distribution of thermal sensitivity were primarily interested in mapping the number of cold and warm spots per square $\mathrm{cm}$ on different skin regions. Interestingly, the overall density of cold spots per square centimeter of human skin was found to be higher than that warm spots (Tab. 1) (151).

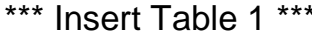

However, while the historical importance of this early works is not questioned, it is important to note that the maps of thermal spots provided little mechanistic information on the nature of the warmth and cold sense and of their regional distribution, particularly due to the difficulties in determining whether this punctate thermal sensibility was the result of discontinuous cold and warm sensitive fields, or whether the same spots represented areas of higher sensitivity as part of a thermosensitive continuum (151). In this respect, more recent investigations have provided mechanistic insights into the psychophysical nature of the warmth and cold sense and of its regional distribution across the human body by mapping both perceptual and physiological differences in regional thermosensitivity.

In a study in which eight different regions of the body (i.e. forehead, scapula, dorsal upper arm, volar forearm, chest, abdomen, lateral thigh and calf) were stimulated (surface area: $22 \mathrm{~cm}^{2}$ ) with different levels of radiative heating (range: 20 to $500 \mathrm{~mW} / \mathrm{cm}^{2}$ ) (274), it was found that regions such as the forehead and cheek were significantly more sensitive to warmth than the (upper and lower) limbs. However, these regional differences were more marked at lower than at higher levels of irradiance. Approaching levels of irradiance closer to threshold for heat pain $\left(>42^{\circ} \mathrm{C}\right)$ was indeed observed to reduce the degree of regional differences in thermal sensitivity to warmth.

In line with these results, a recent systematic analysis of warm and cold temperature thresholds for 13 body regions in male and female individuals representative of young (mean age: $22 \mathrm{yrs}$ ), middle (mean age: $50 \mathrm{yrs}$ ) and elderly (mean age: $75 \mathrm{yrs}$ ) age groups, has shown that thermal sensitivity varies across the body by nearly 100 folds, with the face being the most sensitive region, while the lower extremities (i.e. thigh, calf, sole and toe) the least. Also, and in line with the early cold-sensory spots studies presented earlier, the overall thermal sensitivity of the skin appeared to be greater for cold than for warm temperatures. No gender difference in thermal sensitivity were observed, while similar age-related decrements in the thermal sensitivity of the extremities (hands and feet) were reported for both male and females (273).

Recently, Gerrett et al. (119) have also provided detailed body maps of thermal sensitivity to warmth in both male and females. A similar regional distribution of warmth sensitivity (i.e. with the head being more sensitive than the limbs) as previously observed in both males and females (273), was also observed in this study. However, female participants were observed to present higher absolute warmth sensitivity to local conductive warming $\left(40^{\circ} \mathrm{C}\right)$ delivered by a $25 \mathrm{~cm}^{2}$ thermal probe. Apparently in contrast with the work of Stevens et al. (273), such gender differences in warmth sensitivity could be due to the fact that in Gerrett et al.' study (119), the majority of the observed gender-related differences were located on different areas of the torso, a region which was not explored in details by Stevens et al. (273). It could be therefore speculated that gender-related differences in thermal sensitivity, as observed by Gerrett et al. 
(119), could be localized to specific skin regions rather than being distributed across the whole body.

Overall, what emerges from the studies presented above is that the regional patterns of thermal sensitivity to innocuous warm temperatures (i.e. higher sensitivity of central regions such as the head and lower sensitivity of peripheral regions such as arm and legs) appear to be a consistent human feature, both for male (226) and females individuals (119), and irrespectively of age (273).

In terms of its physiological significance, it could be speculated that the relatively higher sensitivity to warm temperature of the head and face, could carry behavioral implications in the context of maintaining thermal homeostasis. As previously mentioned, human temperature regulation presents an asymmetrical nature: the normal core temperature $\left(-37^{\circ} \mathrm{C}\right)$ is indeed closer to its upper $\left(\geq 40.5^{\circ} \mathrm{C}\right)$ than its lower survival limit $\left(\leq 18-20^{\circ} \mathrm{C}\right)(241)$. In light of this, it would be reasonable to hypothesize that an effective thermosensory system (such as the one the body is provided with) would have evolved in a way which would see the more vulnerable regions to heating (i.e. the head, were the main thermal controller of the body, the hypothalamus, is located) (254) being more sensitive to warm temperatures. The fact that these regional differences in thermal sensitivity have also been observed to modulate physiological and autonomic responses provides further support to this hypothesis. For example, it has been repeatedly shown that, when assessing regional thermal sensitivity in terms of the impact that similar changes in skin temperature of different regions has on local sweating (i.e. an autonomic thermoregulatory response), regions such as the face exert a larger impact on sweating regulation than e.g. the lower $\operatorname{limb}(56,222)$. Nadel et al. (222) have showed that, when the face (area=0.03 $\mathrm{m}^{2}$ ) of healthy individuals was exposed to sudden radiative heating $\left(700 \mathrm{~W} / \mathrm{m}^{2}\right)$, local sweating on the thigh was increased three times more than when heating was applied on the thigh. In line with these results, it has been recently reported that during a mild passive heat stress capable of inducing an increase in local sweat rates, when the face was suddenly cooled, sweating was suppressed to an extent which was 3 to 5 times greater than if a similar area of the e.g. lower limb was cooled to the same extent (56).

Altogether, the perceptual and physiological evidence presented above supports the view that thermal sensitivity to warm temperatures varies significantly across the human body according to a particular pattern, which sees central regions such as the head, being more sensitive to warmth than other central (e.g. torso) and peripheral (limbs) regions.

Similar regional differences, however with different patterns, have been observed on the cold side of the temperature spectrum. As opposed to warm, cold sensitivity appears to be higher on the torso (particularly the abdomen) than on the head (226) and limbs (275). Furthermore, the regional distribution of cold sensitivity has been shown not only to be higher on the torso than e.g. on the limbs (238), but to also vary largely within this region $(99,238)$. For example, cold sensitivity across the torso seems to vary significantly, with regions such as the lateral abdomen and lower back presenting higher sensitivity than upper torso regions (Fig. 3) (99). The body maps pictured in figure 3 present data from one of this author's recent studies (99). In the mentioned study, thermal sensations resulting from the application of a standardized cold stimulus (i.e. contact cooling with a temperature $15^{\circ} \mathrm{C}$ lower than local skin temperature) to several regions of the torso, were observed to vary significantly and independently of local skin cooling. When comparing the body maps of regional differences in local skin cooling (see Fig. 3A) and local thermal sensations (see Fig. 3B), it can be observed that the same cold stimulus induced colder sensations when applied to the lower back than to the lateral chest. This, despite the fact that the application of the same cold stimulus resulted in a smaller decrease in skin temperature of the lower back than of the lateral chest. This observation led to the conclusion that that regional differences in sensitivity to cold could therefore be determined by an heterogeneous integration of thermo-afferent information, which appears to be independent of regional changes in skin temperature $(8,30,226)$. 
Similarly to what proposed for the regional distribution of warmth sensitivity, the heterogeneous distribution of cold sensitivity across the body could also carry physiological significance within the context of thermal homeostasis. For example, the fact that the abdomen presents some of the highest sensitives to cold could be the result of the potentially greater requirements of this body region for thermal protection from cold stress. The fact that local cooling of the abdomen has been shown to induce vasoconstriction of the corresponding underlying gastrointestinal tract, with a potentially significant impact on this organ's function (185), supports the hypothesis for which the higher cold sensitivity of the abdomen might represent a form of evolutionary-acquired thermosensory adaptation aimed at optimizing thermal homeostasis (226). Further evidence in support of the physiological significance of the higher cold sensitivity of the torso (than of the limbs) is provided by the observation that the gasping response to sudden skin cooling, resulting from cold water immersion, is significantly greater when the torso is the region exposed to the immersion (30). As the gasping response (i.e. hyper-ventilatory response occurring at the onset of head-out cold-water immersion) is by definition a non-thermoregulatory response driven by neurogenic drive from cold thermoreceptors (30), the fact that the magnitude of this physiological response is influenced by the skin region exposed to cooling (e.g. greater for the torso than the limbs) indicates that, whether due to a higher density of cold thermoreceptors, or to a greater central influence on thermal sensation, different skin regions present distinctively different thermal sensitivities to cold.

As well as varying across the hairy regions of the body, thermal sensitivity appear to vary between hairy and glabrous (e.g. palm of the hand) skin sites (273). For example, hairy skin has been proposed to be more sensitive to thermal stimuli, while glabrous skin presents higher spatial tactile acuity (230). Such differences in thermal sensitivity have long been suggested to dependent upon potential differences in sensory innervation between hairy and glabrous skin sites. However, recent evidence has indicated that, rather than being determined by physiological differences only (160), previously observed differences in thermal sensitivity between hairy and glabrous skin could have been partly determined by biophysical factors related to the properties of these types of skin (161). As opposed to hairy skin, glabrous skin presents a thicker stratum corneum (i.e. outermost skin layer), a fact which translates in a greater thermal insulation and in a potentially lower thermal conductance of this type of skin as compared to the rest of the body (256). This lower thermal conductance could result in prolonging the time needed for a given change in the temperature of the superficial layers of glabrous skin to penetrate to the underlying tissues (e.g. stratum granulosum), where thermoreceptors are located (206). As a result, given the same short-term contact cooling and/or heating, smaller temperature changes would effectively occur around the site of innervation of glabrous skin thermoreceptors, when compared to the temperature changes occurring around hairy skin thermoreceptors. In turn, this could lead to the apparent conclusion that glabrous skin could present potentially lower thermosensitivity than hairy skin. The hypothesis that differences in thermal sensitivity between hairy and glabrous skin could be partly determined by biophysical factors related to the properties of these types of skin is supported by the findings of a recent study, in which the absence of intrinsically different mechanisms for heat sensitivity between these types of skin was observed when heat stimulation of the dorsum (i.e. hairy) and palm (i.e. glabrous) of human hand, as delivered via non-contact laser stimulation, induced qualitatively and quantitatively similar heat-pain sensations and brain potentials in healthy humans (161). Contrary to laser stimulation, contact heat stimulation of the palm of the hand (performed with a thermal probe) had to be significantly higher than that of the dorsum in order to obtain comparable heat-pain sensations. Furthermore, brain responses (i.e. evoked potentials) to contact heating of the palm presented significantly longer latencies and smaller amplitudes than those resulting from the stimulation of the dorsum, despite the stimulating temperature used for the palm of the hand was higher than that applied to the dorsum of the hand (161). These observations indicated a potential thickness-dependent delay and attenuation of temperature-induced evoked potentials, hence thermal sensations, when conductive (as opposed to laser/radiant) heating was applied to glabrous skin. All in all, such findings highlight the importance of taking into account potential biophysical differences between skin sites when investigating thermal sensitivity across the body. 


\section{Summary of the psychophysical bases of temperature sensation}

When the skin is adapted to temperature values ranging from $\sim 30$ to $\sim 34{ }^{\circ} \mathrm{C}$, neither warm nor cool sensations are experienced. Any linear increase or decrease in skin temperature from this adapting value results in corresponding warm or cool sensations (175), with a temperature threshold which varies according to the rate of temperature change (e.g. the faster the change, the smaller the temperature threshold). On the contrary, when skin temperature is adapted to values below $\sim 30^{\circ} \mathrm{C}$ or above $-34^{\circ} \mathrm{C}$, persisting cool and warm sensations are respectively experienced, with a duration which is longer the more the new adapting temperature deviates for the neutrality range. In this respect, when full adaptation (i.e. absence of warm or cool sensations) has not yet been achieved, any change in skin temperature from these new adapting values will initially result in increases or decreases in the intensity of the persisting sensation. This initial reduction in the intensity of the persisting sensation seems to contribute to an increase in the temperature threshold for detecting changes in skin temperature when this is adapted to values outside the neutrality range (i.e. -30 to $\sim 34^{\circ} \mathrm{C}$ ).

The rate of change in skin temperature affects the way a temperature stimulus is perceived. The faster the increase or decrease in skin temperature from an adapting temperature of $34^{\circ} \mathrm{C}$, the smaller the skin temperature variation required to induce a thermal sensation. On the contrary, the slower the rate of change, the greater the skin temperature variation required to induce a thermal sensation.

An increase in the area of the skin exposed to warming/cooling results in a decrease in the temperature threshold required to induce a thermal sensation $(76,151)$. The interaction between the stimulus area and the degree of change in temperature influence the temperature threshold according to a mechanism of spatial summation $(76,137,173)$, which is similar across the body, but which is also limited by lower (minimum amount of skin temperature variation required to generate thermal sensation) and higher (threshold for heat pain) thermal thresholds (274).

During the contact with a surface with a temperature different form skin temperature, a sudden change in temperature occurs as a result of the heat exchange between the skin and the object touched (14). This resulting temperature change often generates supra-threshold changes in skin temperature whose amplitude has been shown to linearly correlate with the magnitude of the resulting thermal sensation (76).

Regional variations in warm and cold temperature sensitivity exit across the body. The density of cold spots per square centimeter of human skin seems higher than that of warm spots (151). Body regions such as the head (e.g. forehead and cheek) are significantly more sensitive to warmth than the torso and the (upper and lower) limbs. However, these regional differences are more marked at lower than at higher levels of thermal stimulation. As opposed to warm, cold sensitivity appear to be higher on the torso (particularly the abdomen) than on the head (226) and limbs (275). The fact that the regional distribution of warm and cold temperature sensitivity presents asymmetrical patterns is in line with the behavioral and autonomic function that temperatures sensitivity plays in maintaining thermal homeostasis. The human thermosensory system appears therefore to have evolved so that the more vulnerable regions to heating (i.e. the head, were the body main thermal controller, the hypothalamus, is located) (254) present a higher sensitivity to warm temperatures; while the more vulnerable regions to cooling (i.e. the torso, were local cooling has been shown to induce vasoconstriction of the corresponding gastrointestinal tract, which in turn affect the organ's function) (185) present a higher sensitivity to cold temperatures.

At this point, it appears clear that the critical contribution of psychophysical studies to our understanding of human thermoreception lays in the quantitative assessment of the relationship between biophysical changes in skin temperature and resulting thermal sensations. While 
determining the psychophysical principles underpinning our ability to sense temperature has represented an important step in the process of unveiling the mechanisms of human thermoreception, the same has opened to numerous questions on what neurophysiological mechanisms support temperature sensing in humans. How temperature is transduced at a molecular level, and how this is peripherally encoded and centrally processed by our nervous system, in order to provide us with such a finely-tuned thermo-sensory ability, will be explored in the following section.

\section{Neurophysiology of temperature sensation}

In humans and in other non-human primates, non-painful and painful skin thermal sensations are mediated by a variety of primary afferent nerve fibers that encode and transmit thermal information to the central nervous system (261). Specific temperature-activated ion channels are expressed in the terminals of these afferent nerve fibers which end as free nerve endings in the skin $(128,261)$. These sensory neurons encode and transmit thermal inputs which are centrally integrated through the spino-thalamic tract by higher order neural structures located in different regions of the cerebral cortex (62).

The majority of the knowledge on the neurophysiology of skin thermal sensations has arisen from studies performed between the mid-1930 and 1980, which thoroughly investigated the electrophysiological properties and functions of first order thermo-sensory nerve fibers innervating the skin $(74,75,145,147,148,165,166,174,187,313,314)$. Most of these works used the primate and cat as the preferred animal models to electrophysiologically explore the function of mammalian skin thermoreceptors. Along with such experiments, correlative studies with human psychophysical performance were used to infer on the neurophysiological nature of human peripheral thermoreception. While these studies have provided the basis for well-established notions, such as fibers specificity in humans (i.e. the class of specific nerve fibers which are involved in warm and cold temperatures detection), recent evidence based on microneurographic recordings of activity in primary thermo-sensory nerve fibers in awake humans has challenged some of these well-established ideas $(39,40,264)$.

The recent electrophysiological characterization of second order thermo-sensory neurons involved in conveying thermosensory information at a spinal level, in both animal models and humans, has broaden our understanding of the afferent spinal pathways for thermoreception. Along with recent advances in neuroimaging techniques, which have allowed the investigation of the cortical projections of third order thermo-sensory neurons, these studies have provided novel insights on the central processing sub-serving conscious thermal sensation in humans $(60,62$, $68,78,80,92,198,213,251)$.

Finally, one of the most recent advances in our understanding of the neurophysiology of thermal sensations has to do with the discovery and molecular characterization of specific thermosensitive transient receptor potential (TRP) ion channels expressed on first-order thermo-sensory neurons. In light of their role in allowing the transduction of physical temperature into neural signals, these temperature-sensitive proteins have been defined as the putative "thermometers" in our skin (299). Studies investigating the role of thermo-sensitive transient receptor TRP ion channels in thermal sensation have rapidly flourished over the past 20 years. Using mammalian (i.e. mouse) $(3,85,183,213)$ and non-mammalian (i.e. drosophila) $(114,298)$ animal models, these investigations have significantly contributed to our understanding of how temperature is gated at a molecular level. Furthermore, studies endorsing pharmacological manipulation of such channels in humans have recently started to be performed (116), and have shown significant analogies with findings from animal models. Nevertheless, numerous questions on the specificity of these channels and on their role in modulating conscious thermal sensations remain outstanding (255). 
In light of the above, the different levels of integration of skin thermal sensation, along with their anatomical and physiological properties, will be discussed below. Firstly, the properties and functions of first order thermo-sensory nerve fibers innervating the skin will be presented. This analysis will be then followed by a detailed overview of the characteristics of central (i.e. spinal and cortical) thermo-sensory integration. Finally, the molecular properties and temperaturedependence of thermo-sensitive TRP ion channels will be discussed.

\section{Peripheral thermo-receptive nerve fibers}

Temperature sensations resulting from thermal stimulation of the skin are correlated with localized neural structures. From a neurophysiological point of view, these neural structures are described as thermoreceptors, namely free nerve endings excited only or preferably by temperature stimuli (151). Figure 4 depicts a cold thermoreceptors in the glabrous skin of the cat's nose. Free nerve endings with thermo-receptive properties can be categorized in 3 different groups: 1) thermo-receptive fibers selectively excited by either innocuous warming or cooling (i.e. C- and Aס-nerve fibers) $(40,74,75,134,146,184) ; 2)$ nociceptive fibers excited by intense warming and cooling sufficient to elicit pain in man (i.e. polymodal C-nerve fibers) $(134,161,187$, $264)$; 3) low threshold slowly adapting mechano-receptive fibers sensitive to innocuous warming and cooling (i.e $A ß$ - nerve fibers) $(37,90,149,162)$. Both cold and warm sensitive first order afferents present either single or multiple (e.g. 2 to 5) spot-like receptive fields in the skin with diameters of $<1 \mathrm{~mm}(134,146,174,184)$. First order neurons with thermosensory properties are pseudo-unipolar: they present their cell bodies in the dorsal root ganglia and in the cranial ganglia and extend their afferent projection to the skin' epidermal layer (stratum granulosum; where they respond to sensory stimulations) and to the dorsal root of the spinal cord (where they transmit afferent information) (Fig. 4).

Zotterman was the first to describe a thermoreceptor in the tongue of the cat $(313,314)$. Following this early work, investigations of thermoreceptors and afferent nerve fibers have been conducted in non-primates (e.g. cat) $(145,148,162)$ and primates (e.g. macaque) $(74,147,162$, $165,166,174,201)$ animal models as well as in humans $(39,40,107,146,151,165,166,184$, 309). From a neuroanatomical point of view, studies in primates have shown that cold-sensitive fibers appear to belong primarily to the $A \delta$ range, i.e. thinly-myelinated nerve fibers with diameters of $1.5-3 \mu$ and conduction velocities ranging from 3.6 to $15 \mathrm{~m} \mathrm{~s}^{-1}$ (162). Darian-Smith characterized the properties of A $\delta$ cold-sensitive fibers in the median nerve of monkeys, and showed that such thermosensory fibers represented a third of all the A-fibers composing the median nerve. These fibers responded to cooling with conduction velocities ranging from 5 to 25 $\mathrm{m}^{-1}(75)$. As well as Aठ cold-sensitive fibers selectively responding to cooling (146), evidence in primates has indicated that another class of A-fibers, namely $A ß$ mechanoreceptive fibers, also respond to skin cooling $(146,149,162)$. Such fibers show high conduction velocities (30 to 80 $\mathrm{m}^{-1}$ ) and large axonal diameters $(5-13 \mu)(162)$. Along with the electrophysiological characterization of thermo-receptive nerve units in primate models, indirect measurements (i.e. based on reaction time) of nerve conduction velocities in human psychophysical experiments of cold and warm sensory thresholds, have provided evidence for the fact that human cold-sensitive fibers are primarily (if not exclusively) myelinated units in the A-range (i.e. conduction velocities: 2 $\left.-8 \mathrm{~m}^{-1}\right)(107,282,309)$.

As a result of the evidence reviewed above, the notion that myelinated units in the A-range represents the main neuronal population responding to non-noxious skin cooling in humans has often been accepted as conclusive. However, it should be noted that this concept is challenged by the fact that cutaneous $\mathrm{C}$-fibers, unmyelinated nerve fibers with conduction velocities ranging from 0.3 to $2 \mathrm{~ms}^{-1}(74)$, identified in the cat (145), primate (162) and human $(39,40,146,264)$, have been repeatedly shown to respond to skin cooling, with characteristic responses similar to those of primate A-fibers thermoreceptors (162). For example, Campero et al. (40) have recently reported an extensive microneurographic characterization of human cold sensitive C-fibers. 
These fibers were shown to respond to 10 -s cooling pulses (i.e. temperature range of 30 to $15^{\circ} \mathrm{C}$ from a baseline temperature of $35^{\circ} \mathrm{C}$ ) with conduction velocities of 0.8 to $3 \mathrm{~m} \mathrm{~s}^{-1}$ and a with characteristic initial phasic response (lasting 2-3 s), which decayed towards a slowly adapting tonic response (Fig. 5A, B \& E).

Apparently in contrast with previous literature on the selective role of Aס fibers in carrying information about cold stimuli, Campero et al.'s results (40) could be explained by either the possibility for such $\mathrm{C}$-fibers to code thermal information which do not enter the conscious processing of thermal sensation (these could rather sub-serve thermoregulatory functions); or alternatively, by the fact that human afferents are non-uniform in their structure, presenting incomplete myelination distally and complete myelination proximally (40). Recently, and as a potential third hypothesis on the sensory function of cold sensitive unmyelinated C-fibers, it has been proposed that these fibers could sub-serve the burning hot pain experienced when painfully hot or cold stimuli are applied to the skin (39). These fibers could be therefore considered to represent a specific fiber population, namely C2-fibers (39), whose role could be complementary to that of $A \delta$-fibers in characterizing the variability of cold thermal sensations (i.e. non-painful and painful) in humans. The complementarity of C2-fibers in sub-serving thermal sensation is highlighted by the fact that these differ from $A \delta$ cold sensitive fibers with regards to their range of responsiveness and maxima discharge, which appears to be below (i.e. $<10^{\circ} \mathrm{C}$ ) what recorded in Aঠ fibers (Fig. 5C) (39). C2-fibers could therefore carry thermally-induced, quasi-nociceptive information which could be integral to characterize the whole spectrum of thermal sensations in humans (from painful to innocuous thermal sensations) (39). Furthermore, the identification of second-order heat-pinch-cold lamina I nerve fibers in cats and monkeys (see Spinal integration paragraph below for further details), responding to cold stimuli within the activity range of human first order cold-sensitive C2-fibers $(65,135)$, supports the hypothesis for which a quasinociceptive thermal pathway could be present both at a peripheral as well as at central level. The quasi-nociceptive thermal function of cold-sensitive C2-fibers could be therefore integral to the occurrence of phenomena such as paradoxical thermal sensations (e.g. burning upon extreme cooling), as well as to numerous masking mechanisms between A- and C-fibers (e.g. artificially reducing cold sensitivity in humans via anesthesia or compression ischemia, results innocuous skin cooling evoking burning sensations) $(61,203,310)$. Interestingly, recent evidence in mice models of cold sensation has shown that $\mathrm{C}$-fibers seems to predominantly contribute to conscious cold sensations in this animal (213). This observation further supports not only the fact that Cfibers could be predominant cold receptors in sub-primate species (as previously reported in e.g. the cat) (145) but also, that these fibers could conserve an important thermo-sensory role amongst the cold afferent populations in mammalian species, amongst which humans $(65,135)$.

With regards to warm-sensitive nerve fibers, studies in primates $(74,147)$ and humans $(134,184$, 291 ) have shown that warm fibers appear to belong exclusively to the C-range, i.e. unmyelinated nerve fibers with conduction velocities ranging from 0.4 to $2 \mathrm{~ms}^{-1}$. These observations have been confirmed in humans both with indirect measurements of nerve conduction velocities $(107,282$, 309) as well as with direct measurements of neural activity in C-fibers responding to skin warming $(134,184,291)$.

Altogether, the neuroanatomical properties of first order thermosensory nerve fibers can be summarized as follow:

1) sub-primates models (e.g. mouse, cat) have shown that cold and warmth are conveyed primarily by slowly conducting unmyelinated C-fibers; 2 ) primate models have shown that cold is conveyed primarily by fast conducting myelinated $A \delta$-fibers, while warmth is conveyed by slowly conducting unmyelinated $\mathrm{C}$-fibers; 3 ) human experiments have shown that cold is conveyed by both fast conducting myelinated $A \delta$ fibers and by a specific class of slowly conducting unmyelinated C-fibers (i.e. C2-fibers), while warmth is exclusively conveyed by slowly conducting unmyelinated C-fibers; 4 ) cold sensitive $A \delta$-fibers seem to be primarily responsible for innocuous cold sensations while cold-sensitive $\mathrm{C} 2$-fibers seems to be primarily responsible for painful burning sensations associated with both very low and very high temperatures; 5) both cold and 
warm sensitive afferents present either single or multiple (e.g. 2 to 5) spot-like receptive fields in the skin with diameters of $<1 \mathrm{~mm}(134,146,174,184)$.

Despite their different neuroanatomical properties (e.g., fiber type, conduction velocity), both cold and warm thermosensitive fibers present similar distinctive neurophysiological characteristics: 1 ) they have a static discharge at constant temperatures (e.g. 30 to $34^{\circ} \mathrm{C}$ ); 2) they show a dynamic response to temperature changes; 3 ) they are not excited by mechanical stimuli (162). These characteristic responses and properties will be discussed below.

\section{Static thermal sensitivity}

At steady state temperatures, primate $A \delta$-cold fibers have a characteristic stimulus response function which is bell-shaped, with a maximal steady state activity between 20 and $30{ }^{\circ} \mathrm{C}$ and lower activity at lower (i.e. down to $15^{\circ} \mathrm{C}$ ) and higher (i.e. up to $45^{\circ} \mathrm{C}$ ) temperatures $(148,166$ ). Maximum static frequency discharge in these fibers (i.e. $\sim 9$ impulses per sec) occurs at a temperature of $\sim 27^{\circ} \mathrm{C}(148)$. Primate $A \delta$-cold fibers present a characteristic pattern of burst discharge at steady temperatures consisting of short bursts of impulses (i.e. 2 to 12 per sec) separated by silent intervals (162). At maintained temperatures below $17^{\circ} \mathrm{C}$ or above $40{ }^{\circ} \mathrm{C}$, cold fibers maintain a very low frequency discharge or become silent. Recordings of $A \delta$-cold fibers in awake humans have proven difficult to perform. Campero et al. (39) have recently reported microneurographic recordings from two human $A \delta$-cold fibers. It was shown that such fibers responded dynamically to a cooling ramp in the range of 30 to $14{ }^{\circ} \mathrm{C}$ with a maximum discharge at temperatures $\sim 27^{\circ} \mathrm{C}$, and with conduction velocity of $3.8-4.4 \mathrm{~ms}^{-1}$ (Fig. 5C). As that, indicating that human $A \bar{\delta}$-cold fibers present similar characteristics to primate $A \bar{\delta}$ cold fibers.

Paradoxical discharge in primate A type cold fibers in response to heat stimuli (i.e. $>47^{\circ} \mathrm{C}$ ) has been previously reported and shown to be influenced by deep body temperature (201). Heat sensitivity in cold fibers innervating the glabrous skin of macaque monkey has been indeed shown to occur in response to noxious thermal stimuli, and with a heat threshold which decreases as body temperature increases (201). Figure 6D shows paradoxical discharge in primate cold sensitive A-fibers in response to heat stimuli (i.e. $>47^{\circ} \mathrm{C}$ ) and their modulation by different core temperatures. This phenomenon seems to be due to a potential association between hyperthermia-induced increases in skin blood flow in the cold thermoreceptor's field and the receptor's sensitivity to heat stimuli. From a behavioral point of view, the fact that cold-fibers sensitivity to heat stimuli could be increased proportionally to the harmful nature of a thermal stimulus, might indicate the implication of thermal components in pain processing (201). Interestingly, paradoxical discharge in human cold-fibers have also been reported in cold sensitive C2-fibers (Fig. 6A, B \& C) (40), These human fibers present a characteristic burst discharge upon warming to $45^{\circ} \mathrm{C}$, which is similar to what reported in primate A-type cold sensitive fibers.

Warm C-fibers identified in primates have ongoing activity at static temperatures above $30{ }^{\circ} \mathrm{C}$, and this activity vanishes upon cooling $(147,148,162)$. Two classes of warm sensitive fibers have been identified in primates: one group, in which the function of their discharge rate versus steady state stimulus temperature follows a bell-shaped curve, with static activity range between 30 and $44^{\circ} \mathrm{C}$ and an average maximum of $12 \mathrm{imp} / \mathrm{sec}$ at $41^{\circ} \mathrm{C}$; a second group in which the steady frequency increases continuously with rising skin temperature up to $44^{\circ} \mathrm{C}(147)$. Figure 7A shows average static impulse frequency of these two populations of single warm fibers from hairy skin of foot in rhesus monkey as function of constant temperatures. In contrast with primate cold fibers, primate warm fibers present a regular pattern of burst discharge at steady temperatures $(147,162)$, which can however become more irregular at higher temperatures when fibers' activity starts to decrease (147). At maintained temperatures below $30^{\circ} \mathrm{C}$ or above $45^{\circ} \mathrm{C}$, as well as upon sudden cooling, warm fibers maintain a very low frequency discharge or become silent (184). Human warm sensitive C-fibers have been isolated by Konietzny and Hensel (184). 
These warm sensitive fibers showed a static discharge that started near $35^{\circ} \mathrm{C}$ and increased in frequency with rising temperature up to $45^{\circ} \mathrm{C}$ (note: this value was the maximum temperature tested and not necessarily the temperature at which maximum discharge is achieved). In a successive study, Hallin et al. (134) reported similar results, showing human warm C-fibers to differ significantly from polymodal $\mathrm{C}$ nociceptor (i.e. activated by heat and mechanical pressure) (291) with regards to their receptive field, temperature and mechanical sensitivity. Warm C-fibers were indeed reported to: 1 ) have spot-like receptive fields; 2) to fire optimally within the temperature range of innocuous warming (i.e. 30 to $45^{\circ} \mathrm{C}$ ), with a maximum impulse frequency of $\sim 60 \mathrm{imp} / \mathrm{s}$ at $\sim 42{ }^{\circ} \mathrm{C} ; 3$ ) to be insensitive to mechanical stimulation. C-polymodal fibers were on contrary shown to present: 1 ) large and complex receptive fields; 2) increasing firing upon temperature stimulations above $45^{\circ} \mathrm{C}$ (i.e. noxious heat); 3 ) responsiveness to mechanical stimulation (134). Figure 7B shows static thermal sensitivity of these two fibers populations. Altogether, the information available on the steady-state discharge properties of primate and human thermo-receptive fibers well explain psychophysical results on the role of absolute skin temperature on human thermal sensation. The fact that when skin temperature is within the neutrality range (i.e. $30-34^{\circ} \mathrm{C}$ ) neither warm nor cold sensations are experienced, can be explained by the presence of an overlapping steady state discharge at such temperatures in both cold- and warm-sensitive nerve fibers, ultimately resulting in a "neutral" thermal sensation. In line with this, the fact that when skin temperature is adapted to values below or above $\sim 30$ to $\sim 34{ }^{\circ} \mathrm{C}$ (i.e. neutrality range) persisting cold or warm sensation are respectively experienced, can be explained by the observed increase in the steady state discharge of a particular fiber population (e.g. cold-sensitive), with concurrent cross-inhibition of the other (e.g. warm-sensitive), at the new adapting temperature.

\section{Dynamic thermal sensitivity}

Hensel and Boman (146) firstly reported electrophysiological recording from cold sensitive fibers in the superficial radial nerve of awake humans and shown that these fibers respond with a characteristics impulse frequency upon cooling. Similarly, Konietzny and Hensel (184) reported electrophysiological recording from warm sensitive fibers in the superficial radial nerve of awake humans and shown that these fibers respond with a characteristics impulse frequency upon warming. Irrespective of the initial temperature, cold sensitive fibers were always observed to respond with an initial overshoot of their discharge to sudden cooling. On the contrary, upon transient warming, cold receptors discharge underwent transient inhibition (Fig. 8). A similar pattern (however in the opposite direction) was reported for warm receptors (184). Human warm and cold sensitive fibers appear therefore to be particularly sensitive to rapid changes in skin temperature.

Neurophysiological evidence arising from animal studies has indicated that sudden changes in skin temperature determine dynamic responses in first order thermosensitive nerve fibers by influencing three main parameters of fibers' activity, namely: 1) peak discharge frequency; 2) rate of frequency increase; 3) cumulative impulses during stimulation.

Studies in primates have shown that fibers' peak discharge frequency is dependent upon the magnitude as well as the rate of temperature change. Indeed, peak frequency increases with stimulus intensity and with the rate of change in temperature. As a result, greater changes with faster rates induce higher peak discharge frequencies and faster adaptation, while slower rates of change induce lower peak discharge frequencies and longer adaptation (174). Such behavior can be observed in figure 9A. Accordingly, the cumulative impulses during the stimulation are dependent upon the magnitude and rate of temperature change. Indeed, cumulative impulses are higher with greater stimulus intensity and with slower rates of change in temperature. Finally, the rate of frequency increase appears to be the same regardless of the intensity of the change (at least within a $0-8{ }^{\circ} \mathrm{C}$ temperature change), and within the first 0 to $4 \mathrm{~s}$ of stimulation (Fig. 9B) (74). 
At this point, an important concept for understanding how the dynamic activity of first order thermo-sensory neurons contribute to thermal sensation, is related to identifying the parameter (i.e. peak frequency; the cumulative impulses during stimulation; the rate of frequency increase) that might be used by the nervous system to determine the intensity of the stimulus. As the rate of frequency increase appears to be the same regardless of the intensity of the temperature change, this implies that this parameter is not the main one signaling the magnitude of temperature change. It can be therefore extrapolated that peak discharge frequency and cumulative inputs play a primary role in temperature coding. That these parameters determine the intensity and timing of resulting thermal sensations has been supported by both psychophysical and neurophysiological evidence.

Let's take the following example. Psychophysical evidence indicates that the rate of change in temperature does not affect the maximum intensity experienced for a given temperature stimulus, but in fact this influences only the time at which such maximum is experienced. Accordingly, a temperature stimulus $5^{\circ} \mathrm{C}$ lower than skin temperature, applied at a rate of $2{ }^{\circ} \mathrm{C} / \mathrm{s}$, is experienced as cold as a temperature stimulus $5^{\circ} \mathrm{C}$ lower than skin temperature applied at a rate of $0.5{ }^{\circ} \mathrm{C} / \mathrm{s}$. However, as the rate of change in temperature determines the time at which the maximum intensity of the stimulus is experienced $(151,175)$, a temperature stimulus $5^{\circ} \mathrm{C}$ lower than skin temperature, applied at a rate of $2{ }^{\circ} \mathrm{C} / \mathrm{s}$, will be experienced at its maximum sooner (i.e. after $2 \mathrm{~s}$ from application), than when the same stimulus is applied at a rate of $0.5^{\circ} \mathrm{C} / \mathrm{s}$ (i.e. this will be experienced at its maximum after $10 \mathrm{~s}$ of application) (174). Neurophysiologically, such observation is explained by the fact that the same temperature stimulus $5^{\circ} \mathrm{C}$ lower than skin temperature will initially induce a higher peak frequency when applied at a rate of $2{ }^{\circ} \mathrm{C} / \mathrm{s}$ than at $0.5^{\circ} \mathrm{C} / \mathrm{s}$. However, when applied at $2{ }^{\circ} \mathrm{C} / \mathrm{s}$, this stimulus will also generate cumulative impulses which will be lower than when the same is applied at $0.5^{\circ} \mathrm{C} / \mathrm{s}$. As a result, the two parameters (i.e. peak discharge frequency and cumulative inputs) seem to balance up in giving the same magnitude estimate (i.e. perception of the absolute intensity of the stimulus), however at different times (i.e. longer for the slower rate of change). In support of this observation, psychophysical evidence has repeatedly shown that the slower the rate of change (i.e. lower peak frequency), the greater the skin temperature variation required (i.e. greater cumulative inputs) to induce a thermal sensation $(151,175)$. All in all, this evidence indicates that the interaction between peak frequency and cumulative inputs is the likely parameter used by the nervous system to determine the magnitude of temperature changes.

The dynamic properties of first order thermoreceptive fibers help explaining other psychophysical phenomena, such as why at adapting skin temperatures outside the neutrality range (i.e. $<30-$ $34^{\circ} \mathrm{C}<$ ), sudden changes in skin temperature are initially perceived as to reduce the initial persisting thermal sensation (e.g. stimulus perceived as less cold), before inducing new thermal sensations (e.g. warm sensation) aligned to the direction of the temperature change (e.g. increase in temperature). In this particular case, changes in skin temperature from adapting values outside the neutrality range (e.g. $<30-34^{\circ} \mathrm{C}$ ), would indeed initially reduce activity in cold fibers before reaching the level of maximal activation of warm receptors. This, along with the fact that warm sensitive fibers seem to have a lower peak frequency response and cumulative impulses to sudden temperature changes when adapted to temperatures below the thermoneutral range (74), could therefore explain why outside thermal neutrality, sudden changes in skin temperature are not immediately experienced as the sensation expected for the resulting direction of temperature change, but in fact, these are experienced as a reduction in the intensity of the opposite thermal sensation. Finally, the information available on the dynamic discharge properties of primate and human thermo-receptive fibers well explain psychophysical results on the role of the rate of change in skin temperature on human thermal sensation. Indeed, the fact that thermal detection thresholds are decreased with faster rates of temperature change $(74$, 174), is explained by the fact that faster, as opposed to slower, changes in skin temperature induce higher transient discharge frequency of thermoreceptive fibers.

Apart from the static and dynamic responses to thermal stimuli in single fibers, what is also of interest is how the simultaneous activation of different fibers in response to a thermal stimulus is 
integrated. Psychophysical studies have shown that the information provided to the central nervous system by the activation of a single fiber would be indeed insufficient to justify the discriminative capacity of human subjects (166). Furthermore, in realistic conditions, thermal stimulation often activates thermo-receptive fibers populations, rather than a single fiber. In this respect, Johnson et al. (166) calculated the minimum amount of active fibers required to match human performance, as described by the ability to discriminate between two successive cold stimuli at different intensities. To match human performance, it was shown that an ideal observer, who base its thermal discriminability (or difference limen) on averaging the impulses obtained by each single fiber activated by the cold stimulus, would require the activation of at least 16 first order cold-sensitive fibers (note: this was based on neurophysiological recordings in monkey) to be able to discriminate between successive cooling pulses of different intensities (166). A similar approach was also used to define the amount of sensory inputs required by the brain to discriminate between warm stimuli of different intensities (74). Specifically, this approach consisted in comparing the responses evoked by warming pulses in the population of warm fibers engaged by a warming stimulus, with behavioral responses from animal and humans discriminating between thermal pulses (74). As a result of such observation, it appears that the thermal information relayed by all warm fibers engaged by a warm stimulus is combined centrally by a simple additive or averaging process, where each fiber's response is given equal weighing in the integrative process. This process would retain enough information to account for intensity discrimination in humans (74).

In summary, the body of literature reviewed so far provides supporting evidence on the properties and functions of first order thermo-sensory nerve fibers innervating human skin. The temperaturedependent neural activity of these first-order sensory neurons represents the primary mechanism which allows our central nervous structures to convert physical changes in skin temperature into conscious thermal sensation. In the next section, an overview of the central structures involved in coding the thermal inputs conveyed via peripheral thermoreceptors into conscious thermal sensations, and of how such integration is operated, will be presented.

\section{Central mechanisms of thermal integration}

A detailed account of the neuroanatomy of the central nervous system is not the object of the present review, and for more information on this subject, the reader is referred to the reference textbook edited by Kandel et al. (169). Nevertheless, in order to facilitate the understanding of the central mechanisms involved in temperature sensation, a brief overview of the basic neuroanatomical characteristics of sensory pathways within the central nervous system will be presented.

From a neuroanatomical point of view, sensory pathways within the central nervous system present specific and structured levels of integration which help connecting the peripheral (i.e. peripheral nerves and ganglia) to the central neural structures (i.e. spinal cord, brain stem, subcortical and cortical brain regions). Sensory pathways contain a series of rely nuclei (i.e. clustered neuron cell bodies) and each nucleus has several function and subdivisions. Functionally organized neurons are arranged within these pathways, which often cross from one side of the central nervous system to the other at different levels of the neuroaxis.

Afferent fibers from the trunk and limbs enter the spinal cord via the dorsal horns. In a crosssectional view of the spinal cord, the presence of the characteristic $\mathrm{H}$-shaped gray matter, divided into 3 functionally distinct regions (i.e. dorsal, intermediate and ventral) comprising 10 laminae (i.e. layers), can be observed. These laminae contain sensory (dorsal) and motor (ventral) cell bodies. Laminae of grey matter are surrounded by white matter containing ascending (sensory) and descending (motor) bundles of axons, which are organized into dorsal, lateral and ventral columns. Dorsal columns contain ascending sensory axons which convey mechano-sensory information related to touch and tactile stimulation of the skin; lateral and ventral columns contain both ascending and descending bundles of axons which convey 1) sensory information related to 
pain and temperature to higher neural regions (i.e. ascending axons); and 2) motor commands to muscles (i.e. descending axons) (27). Nerve fibers innervating the face and the head enter the spinal trigeminal tract via the trigeminal dorsal horns (63). Fascicles of axons within the spinal cord are organized in a somatotopy way: fibers originating in the lower body enter the dorsal horns and ascend within columns of white matter more dorsally, while fibers originating in higher regions of the body (e.g. upper limbs) enter the dorsal horns and distribute more laterally within the columns. At the cervical level of the spinal cord, where the majority of ascending nerve fibers is present, nerve afferents from the lower body distribute more medially, while afferents from the upper body distribute more laterally in the dorsal and lateral columns respectively. Due to a higher number of ascending and descending axons, the superior regions of the spinal cord present a higher distribution of white matter than the inferior regions.

The thalamus is an important sub-cortical rely and modulation site of sensory information travelling from the spinal cord to the cerebral cortex. This sub-cortical rely presents a number of nuclei, which absolve the function of both receiving sites of afferent projections from specific ascending axons, and originating sites of connections to specific areas of the cerebral cortex.

Finally, as the primary neural center for processing conscious sensory information, the cerebral cortex represents the terminal target for the projections of third order sensory neurons originating in the thalamus. Similarly to the spinal cord, the cortex is also somatotopically organized, with specific areas (e.g. somatosensory one) receiving organized projections from different regions of the body.

The ascending thermosensory pathway in humans presents levels of neuroanatomical and functional organization (i.e. spinal, thalamic and cortical) which are aligned to the general overview of afferent sensory pathways outlined above (169). However, and as opposed to other sensory pathways such as the mechanosensory one, it is not until recently that the mechanisms underpinning the central processing of non-noxious thermal sensations have started to be unveiled (88). In this respect, recent studies on the mechanisms of central thermoreception, which have endorsed both animal models as well as direct evaluations in humans, have started to shed light on the spino-thalamic and thalamo-cortical pathways involved in temperature sensation in humans. The available evidence on the neurophysiological mechanisms which allow temperature stimuli to be integrated at spinal, thalamic and cortical levels, will be presented below.

\section{Spinal integration}

Historical observations on the sensory effects of lesions of the human spinal cord provided initial insights on the potential ascending sites of second order thermo-sensory neurons within the human spinal cord $(29,271,278,304)$. However, due to challenges of examining neurophysiological function in humans in vivo, most of our current knowledge on the neuroanatomy and neurophysiology behind the integration and transmission of peripheral thermosensory inputs within the human spinal cord arises primarily from research based on animal models (i.e. cat and monkey) (306). Nevertheless, more direct evidence in humans has recently become available (159), allowing for a better comparison of results between non-human and human models. Hence, in light of the relevance of mammalian models (particularly cats and monkeys) of thermosensory function in the context of understanding neurophysiological function in humans, and due to the lack of extensive systematic examinations of human spinal integration in vivo, the paragraphs below will present the most representative findings from animal model of spinal thermosensory integration. These will be also integrated with the available evidence from human models and comparative studies.

Historically, human second order thermo-sensory neurons have been hypothesized to ascend the spino-thalamic tract via its anterolateral columns and to terminate in the thalamus. Support for the role of the anterolateral spino-thalamic tract in carrying afferent information via second-order thermo-sensitive neurons derived from early observations of the effects that hemi-section of the human spinal cord produced on somatosensory function (29). Indeed, hemi-section of the human 
spinal cord would often result in impairments in thermal sensation on the side of the body contralateral to the spinal lesion (note: such observation also pointed to the possibility that thermosensitive fibers could decussate to the contralateral side of the spinal cord immediately after entering the dorsal horns) (29). These early observations were further strengthened by studies showing that patients treated with anterolateral cordotomies to cure intractable pain, subsequently developed contralateral thermo-anesthesia $(271,278,304)$.

Following these early observations in humans, neurophysiological support for the presence of second order neurons in the mammalian spinal cord specifically responding to innocuous thermal stimulation of the skin, was firstly provided by Christensen and Perl (49), who identified such neurons in the dorsal horn's margin of the cat's spinal cord. The thermosensitive neurons identified in this animal model were shown to selectively respond to both cutaneous warming and cooling, and to cease their tonic discharge upon opposite thermal stimulation (e.g. skin warming suppressed discharge in fibers specifically responding to cooling). Second-order fibers responding to innocuous cooling were also observed to respond to noxious heat $\left(>45^{\circ} \mathrm{C}\right.$ ) (in line with the previously reported paradoxical discharge at high temperatures of first order cold sensitive thermoreceptors) $(201,315)$, and to intense mechanical stimulation. As thermosensitive second order neurons appeared to receive projections from first-order myelinated $A \delta$ and unmyelinated $\mathrm{C}$-fibers (i.e. cutaneous thermoreceptors), these results provided the first evidence for the fact that the dorsal horn marginal layer of the cat' spinal cord (i.e. Rexed's lamina I) could represent the first specialized sensory nucleus within the ascending mammalian thermosensory pathway (49). Second order thermo-sensitive neurons identified in the cat' spinal cord also showed evidence of both excitatory convergence from several primary afferent fibers, as well as inhibitory inputs from regions near to the excitatory field $(49,305)$. These observations therefore supported the view that a certain level of central integration would already occur at a spinal level. Finally, similar responses as the ones observed in second order thermosensitive neurons (e.g. monotonic response which increases with stimulus intensity), have also been reported in heatsensitive nociceptive second order units (248). Based on the above, it followed that the properties of mammalian thermally-sensitive second-order neurons would resemble (at least partially) activity in first order skin thermoreceptors.

The initial identification and characterization of specific thermo-sensitive second order neurons within the cat' spinal cord opened up questions about 1) what ascending spinal pathway contained the afferent projections of these neurons; and 2) what regions of the mid-brain would be targeted projections of these afferent fibers. In this respect, Craig et al. have recently performed an extensive and systematic characterization of second order thermo-sensitive neurons in the spinal cord of both cats and monkeys $(5,63-65,88,135)$. These investigations based on non-human models have contributed to determine that a specific class of thermosensitive spino-thalamic neurons: 1 ) is located in the lamina I of the spinal cord's dorsal horns; 2) ascends contralaterally in the middle of the lateral funiculus of the spino-thalamic tract; 3) contributes to thermosensory behavior and thermal sensation in those mammals. Hence, the nonhuman-based evidence from Craig et al.'s work $(5,63-65,88,135)$ has highlighted the pivotal role of antero-lateral spino-thalamic integration in mammalian thermo-sensation.

Studies of lamina I thermosensitive neurons identified in the dorsal cap at the apex of the dorsal horn in the cat's lumbosacral regions (Fig. 10A) (65), have provided specific insights on the function of mammalian second order thermo-sensitive spino-thalamic neurons. Cold sensitive fibers in the cat' spino-thalamic tract have been shown to: 1$)$ have ongoing activity $(\sim 3.3 \mathrm{imp} / \mathrm{s})$ at normal ambient temperatures; 2) respond to cooling stimuli as low as $\sim 10^{\circ} \mathrm{C}$ with a stimulusresponse function which sees the discharge frequency to increase with decreasing temperatures (Fig. 10B) (65); 3) present conduction velocities of $5.6 \mathrm{~m} / \mathrm{s}$; 4) present saturation of their discharge at temperatures below $\sim 15^{\circ} \mathrm{C}(65)$. Interestingly, in those studies, cat' second order neurons did not show the characteristic temperature-dependent bell-shaped activity which is observed in first order afferents. As different second order neurons within the same cold-fibers population were observed to respond to skin cooling at different temperature thresholds with peak discharge occurring at different low temperatures, spinal convergence of inputs from different primary afferents was therefore proposed as a potential mechanisms to explain such differences 
between peripheral and spinal responses (65). This hypothesis was strengthened by the fact that cat's lamina I neurons presented larger skin receptive fields than those of primary skin afferents. In terms of their morphological structure, cat's cold-sensitive lamina I cells have been described as pyramidal cells (i.e. neurons of triangular shape with 3 major dendritic poles and 3 to 4 dendrites) (Fig. 10C) (135).

It appears necessary at this point to mention that, along to such specialized thermally-sensitive neurons, other two type of physiologically and morphologically defined classes of second order sensory neurons have been recently identified within the cat' spino-thalamic tract, namely the fusiform nociceptive and the multipolar heat-pinch-cold neurons (135). As cold-sensitive lamina I neurons present specific anatomical and physiological characteristics which distinguish them from nociceptive afferents and from heat-pinch-cold afferents $(64,135)$, it is now accepted that three main categories of lamina I spino-thalamic neurons are present within the mammalian spinothalamic tract, namely: 1) innocuous thermo-receptive specific; 2) nociceptive specific; 3) heatpinch-cold specific (Fig. 10C) $(65,135)$. The importance of such discovery lays on the fact that the properties of the multipolar heat-pinch-cold cells identified within the cat' spino-thalamic tract (135) resemble those of the first order cold-sensitive C2-fibers recently identified in humans to 1 ) respond to severe skin cooling $\left(<10^{\circ} \mathrm{C}\right)$ and 2$)$ to (potentially) contribute to the burning quality of cold pain (see Peripheral thermo-receptive nerve fibers paragraph) $(40,264)$. Accordingly, it is reasonable to hypothesize that in mammals (amongst which humans) both first order coldsensitive C2-fibers and heat-pinch-cold sensitive lamina I second order neurons could lay within a specific afferent pathway, which could convey those sensory inputs underpinning the burning sensations experienced during painfully cold stimulation of the skin.

The characterization of the properties and role of second order heat-pinch-cold neurons in the cat' spinal cord, and of their concurrent responses to heat, cold and painful stimuli, has also contributed to unveiling the neural mechanisms (61) behind a particular psychophysical phenomenon which has long been known in humans, known as the thermal grill illusion (or Thunberg's illusion) (287). According to this phenomenon, when alternated interlocking bars maintained at innocuous warm $\left(40^{\circ} \mathrm{C}\right)$ and cold $\left(20^{\circ} \mathrm{C}\right)$ temperatures are simultaneous touched by human subjects, a burning hot sensation is consistently reported. This, despite the fact that warm and cold bars present temperatures well within the thresholds for heat $\left(\sim 42^{\circ} \mathrm{C}\right)$ and cold pain $\left(\sim 15^{\circ} \mathrm{C}\right)$. Interestingly, the thermal grill-induced burning sensations resemble those experienced during exposure to cold pain (61).

By investigating the thermal grill illusion psychophysically (in humans) as well as with direct recordings of cat's lamina I cold-sensitive and heat-pinch-cold sensitive neurons' activity during thermal grill stimuli, Craig et al. (61) have shown that 1) innocuous cold stimuli alone concurrently activate cold-sensitive and heat-pinch-cold sensitive second order neurons; and that 2) the greater activity recorded in cold-sensitive fibers during exposure to innocuous cold temperatures prevails in characterizing the resulting perception as an innocuous cold sensation. However, it was also observed that when concurrent innocuous warm and cold stimuli were applied to the skin (i.e. condition of thermal grill), activity in cold-sensitive fibers is significantly reduced (potentially due to inhibition dependent on the concurrent activation of warm sensitive C-fibers), while activity in heat-pinch-cold sensitive second order neurons is unaltered. It was therefore concluded that burning hot sensation are experienced as a result of innocuous temperature stimulation during the thermal grill, due to a change in balance between activity in cold-sensitive and heat-pinch-cold sensitive second order neurons, which sees the warm sensitive C-fibersinduced inhibition of cold-sensitive second order neurons unmasking the activation of heat-pinchcold sensitive neurons (61). Altogether, such results provided evidence for: 1 ) the masking role that cold-sensitive lamina I neurons play on heat-pinch-cold sensitive second order neurons under innocuous thermal stimulation; 2) the fact that a decrease in the activity of cold-sensitive neurons under natural conditions, as observed when e.g. skin temperatures drop below the threshold for cold pain $\left(15^{\circ} \mathrm{C}\right)$, result in a greater contribution of heat-pinch-cold sensitive second order neurons to the sensation of burning pain upon severe skin cooling. Unmasking the activity of heat-pinch-cold by removing the masking performed by cold-sensitive fibers implies that under normal conditions, innocuous cold stimuli could potentially inhibit the processing of central pain. 
Everyday experience reminds us how potentially used to such concept we could be. Treating a sore body part with ice to relief pain is indeed commonly used as a practical pain-killer in many cultures. On a more conceptual note, such results also indicate how intrinsically linked the temperature and pain sensing pathways could be $(62,67)$.

In line with the above presented direct neurophysiological recording in cats, further evidence on the role and function of specific second order thermo-sensitive spino-thalamic neurons has arisen from studies performed in primates. For example, in a seminal study, Craig et al. (88) have provided evidence for the presence of specific cold-sensitive second order neurons, which were located in the lamina I of the monkey's spinal cord, and which projected to the ventral medial nucleus of the thalamus via the spinothalamic tract. Such neurons were observed: 1) to be spontaneously active at normal skin temperature; 2 ) to vigorously respond upon cooling (although, contrary to what reported for monkey's primary cold afferents, no apparent bursting discharge was observed in these second-order neurons); 3) to decrease their activity upon sudden warming; 4) to be insensitive to mechanical stimulation; 5) to present conduction velocities of $\sim 8 \mathrm{~m} / \mathrm{s}$ (Fig. 11). The functional characteristics of second order thermo-sensitive neurons as identified by Craig et al. (88), resembled those of first order afferents, particularly in terms of their static and dynamic responses. However, contrary to first order thermoreceptors, monkey' second order thermo-sensitive neurons did not present a bell-shaped decrease in static firing at temperatures below $20^{\circ} \mathrm{C}$. This observation indicated that convergence of signals also from those cold-sensitive first order afferents whose activity peaked below a $\sim 20^{\circ} \mathrm{C}$ temperature threshold, could indeed occur at a spinal level. The hypothesis that spinal convergence from first order afferents could occur in second order neurons was further supported by two other observations. Firstly, as opposed to first order afferents, monkey' second order neurons lack a burst discharge, a fact which would indicate that second order neurons could integrate potentially asynchronous activity in primary afferents. Secondly, monkey's lamina I thermo-sensitive neurons present skin receptive fields which are larger than those of their respective first order thermoreceptive afferents (88), a fact which would indicate integration of inputs from multiple first order units.

The knowledge resulting from the characterization of the properties and function of lamina I coldsensitive second order neurons in both cats and monkeys $(5,63-65,88,135)$, has been recently integrated with evidence supporting the existence also of a specialized spinal pathway for warm discrimination in the cat (5).

Andrew and Craig (5) have identified second order warm-sensitive spinal neurons in the cat' spino-thalamic tract, and reported these fibers to: 1) have conduction velocities in the range of $1.5-3 \mathrm{~m} / \mathrm{s}$ (indicating that these fibers are unmyelinated afferents); 2 ) have very low ongoing activity at ambient temperatures $(\sim 0.22 \mathrm{imp} / \mathrm{s}) ; 3)$ receive inputs for warm-sensitive first order Cfibers (presenting conduction velocities of 0.8-1.2 m/s). Altogether, these properties appeared to well match those of warm-sensitive first order neurons (88). With regards to the temperaturedependence of these neurons' responses, second order warm-sensitive spinal afferents were shown to respond to sudden increases in skin temperature (average threshold: $36.1^{\circ} \mathrm{C}$ ) with a brief phasic (dynamic) response followed by adaptation. At supra-threshold temperatures, warmsensitive spinal neurons presented sustained (static) firing. Interestingly, such fibers did not seem to encode noxious heat stimuli, as their responsiveness was shown to linearly increase from temperatures between 36.1 and $42.7^{\circ} \mathrm{C}$, and to plateau at noxious temperatures (range: $45-53^{\circ} \mathrm{C}$ ) (Fig. 12) (5). The fact that these second order neurons did not show a decrease in their firing with raising skin temperatures above the heat pain threshold $\left(\sim 43^{\circ} \mathrm{C}\right)(5)$, indicated that such fibers could receive afferent projections from both types of warm-sensitive first order thermoreceptors (i.e. those which have been shown to present a bell-shaped stimulus-response function with activity range between 30 and $44^{\circ} \mathrm{C}$; and those which present a linear increase in frequency with rising skin temperature up to $\left.44^{\circ} \mathrm{C}\right)(147)$. Such responses therefore provided evidence for the fact that spinal convergence occurs not only in the cold, but also in the warmth sense.

At this point, while it is understood that the neurophysiological evidence presented thus far on mammalian spinal thermosensory integration is entirely based on non-human models, is important to highlight that these results well match human thermal sensibility performance (5), 
suggesting that common neuroanatomical structures and neurophysiological mechanisms of thermal sensibility could be shared between those species. In this respect, evidence for a specific, slowly conducting, spino-thalamic pathway for warmth discrimination in humans, which would be similar to the one observed in cats, and which could rely on afferent information conveyed by warm-sensitive second order unmyelinated C-fibers, has been recently provided for the first time (159). In a recent study, healthy awake humans have been reported to experience conscious warm sensations, upon delivery of $\mathrm{CO}_{2}$ laser stimuli over the vertebrae column, inducing a brief, laser-dependent increase in skin temperature (up to $39^{\circ} \mathrm{C}$ ). Estimation (via laserevoked brain potential) of conduction velocity in the spinal fibers conveying such sensations revealed an average conduction velocity of $2.3 \mathrm{~m} / \mathrm{s}$, indicating that such fibers belonged to the unmyelinated C-fibers group. Although indirect, this evidence has therefore supported the hypothesis that a specific spino-thalamic pathway for the sense of warmth exists in humans and that this could present similar characteristics (e.g. inputs conveyed by unmyelinated C-fibers with conduction velocity of $2.3 \mathrm{~m} / \mathrm{s}$ ) to those observed in the cat's warm-sensitive pathway (e.g. inputs conveyed within unmyelinated C-fibers with conduction velocity of $1.5-3 \mathrm{~m} / \mathrm{s}$ ) (5).

In summary, from a neuroanatomical point of view, results from animal studies, along with clinical and non-clinical evidence in humans, have indicated that first order neurons with thermosensitive properties innervating the trunk and limbs, enter the spinal cord's dorsal horns and synapse with second order neurons in the lamina I (apex cap of the grey matter). Lamina I second order neurons responding to cooling and warming decussate their ascending projections to the contralateral, antero-lateral quadrant of the spinal cord, and ascend via the antero-lateral columns to the thalamus. Such pathway constitutes the afferent thermosensory spino-thalamic tract. From a neurophysiological point of view, the properties of spino-thalamic thermosensory fibers, as characterized in cats and monkeys, well explain stimulus-response properties and thermosensory performance in humans (65). For example, lamina I cold-sensitive neurons in the cat present a linear increase in their response to decreasing skin temperatures in the range of 34 to $15^{\circ} \mathrm{C}, \mathrm{a}$ fact which is well matched by human psychophysical results on thermosensory magnitude estimation (102). Also, conduction velocities of cat and monkey' spino-thalamic cold-sensitive myelinated neurons ( $\sim 8$ and $\sim 5.6 \mathrm{~m} / \mathrm{s}$ respectively) $(65,88)$ resemble estimated conduction velocities $(-10 \mathrm{~m} / \mathrm{s})$ of cold-sensitive myelinated fibers in the human spino-thalamic tract $(168$, 249). Finally, cat' spino-thalamic warm-sensitive unmyelinated C-fibers (conduction velocities: $1.5-3 \mathrm{~m} / \mathrm{s}$ ) (5) resemble estimated conduction velocities of unmyelinated warm-sensitive C-fibers in the human spino-thalamic tract $(-2.2 \mathrm{~m} / \mathrm{s})(159,168,249)$.

Altogether, the primarily animal-based findings reviewed above provides evidence in support of the role and properties of the spino-thalamic tract as both the main spinal pathway as well as the first level of central integration of thermo-afferent information within the central nervous system of mammals amongst which humans. The successive levels (i.e. thalamic and cortical) of central thermo-afferent integration and their respective neuroanatomical and neurophysiological properties will be reviewed below.

\section{Thalamic integration}

As a second rely of thermosensory information within the central nervous system, the thalamus has been repeatedly shown in the cat $(5,63-65,135)$, primate $(31,88,91)$ and human $(60,66$, $80,194,195)$, to receive projections from lamina I second order neurons conveying thermosensitive information via the spinothalamic tract. Similarly to the evidence reviewed on spinal integration, the majority of the knowledge on the neurophysiology of thalamic thermosensory function is based on studies endorsing animal models (i.e. cat and primate). The importance of such investigations reside on the comparative nature of their findings, as the thalamic regions identified to respond to thermal stimulation in those mammals are homologous to specific regions in the human thalamus. Recently, human studies endorsing thalamic microstimulation during stereotactic surgery in patients with pain and movement disorders $(75,186$, 187), as well as studies assessing thermo-sensory abnormalities and central pain in stroke patients with constrained thalamic lesions (171), have also provided more direct evidence on the 
neuroanatomy of the thalamic regions involved in human thermosensory function. In light of the relevance of mammalian models for understanding human thalamic thermosensory integration, an overview of findings from cat and primate models will precede the analysis of human evidence.

In the cat, lamina I thermosensitive neurons have been shown to project (via the middle lateral funiculus) to the sub medial nucleus in the thalamus, as well as to the ventral aspect of the ventral medial nucleus (VM) and to the dorsomedial aspect of the ventral posterior medial nucleus (VPM) $(63,229)$. Amongst these nuclei, the ventral aspect of the ventral medial nucleus (VM) appears to be of primary importance for thermosensory discrimination. Selective lesions of such region have been indeed shown to significantly impair thermal sensibility in behaving cats (229). However, and in contrast with the large thermosensory deficiency that often results from lesions of the spino-thalamic tract (231), localized lesions in the thalamic projections of cat's lamina I thermosensitive neurons do not always induce complete loss of thermosensory function in this animal (229). This observation would therefore indicate that spino-thalamic projections from lamina I neurons (and related thermosensory information) might not be exclusively conveyed to the cat's thalamus, and that other regions of the brain stem (e.g. parabrachial nucleus) could also contribute to central processing of thermal inputs in this mammal (229). In this respect, the parabrachial area, a region of the cat's mid-brain involved in the control of the homeostatic condition of the body (i.e. interoception) $(67,68)$, has been also suggested to contribute to central thermal processing in the cat (229). Altogether, the evidence above could support the hypothesis that the different regions of the cat's forebrain potentially involved in processing thermal information, could sub serve the integration of different components (e.g. affective and discriminative) of the same thermal stimulus (251).

The identification of the role of the ventral aspect of the ventral medial nucleus (VM) of the cat's thalamus in thermosensory integration is relevant in light of the fact that this region appears to be a primordial homologue of the much larger posterior ventral medial nucleus (VMpo) of the primate and human thalamus. Interestingly, in both primate and humans, this thalamic region (i.e. VMpo) has been observed to also receive thermosensory projections from second order lamina I thermosensitive neurons $(21,60)$. Before reviewing the most recent insights in support of the role of the posterior ventral medial nucleus (VMpo) of the primate thalamus in thermosensory integration, it appears important to present earlier findings on primate thalamic thermosensory integration, which had pointed to the involvement of other thalamic nuclei in primate thermal sensibility. This premise appears necessary in order to provide the reader with a better understanding of how knowledge in this area has developed.

Historically, early studies in the macaque had indicated that the principal terminations of both spino-thalamic thermo-sensitive afferents and medial lemniscus mechano-sensitive afferents occurred in the ventral posterior lateral nucleus (VPL) of the primate thalamus (27). Afferents from spino-thalamic and trigemino-thalamic sensory neurons which terminated in this region of the macaque's thalamus were shown to synapse with third-order neurons, whose projections appeared to target the primary somatosensory cortex (31). These early results on the similarities between projections of both thermo-sensitive and mechano-sensitive afferents supported the longstanding (and potentially incorrect) view that temperature sensibility would only represent a sub-class of touch perception. As well as the ventral posterior lateral nucleus (VPL), early studies had also reported the ventral posterior medial nucleus (VPM) of the macaque thalamus to be involved in conveying thermosensory information important for thermal discrimination (91). Following inactivation of this thalamic nucleus (and related neuronal activity) by lidocaine injection, behaving monkeys were indeed shown to present significantly impaired, although reversible, cold-detection ability (31) (91). However, and as also observed in cats (229), the disruption of the ventral posterior medial nucleus (VPM) of the thalamus did not result in a complete thermosensory deficit (31), indicating that such thalamic nuclei (i.e. VPL and VPM) were not the only sites integrating and processing thermal discrimination in the primate thalamus.

In this respect, more recent studies in primates have indicated that lamina I thermo-receptive units seem to project specifically to the posterior ventral medial nucleus (VMpo) of the macaque's thalamus, where somatotopically organized clusters of specific cold-sensitive neurons have been 
identified $(60,88)$. Cold-sensitive neurons in the posterior ventral medial nucleus (VMpo) of the macaque's thalamus seem to terminate their projections in the insula, a cortical region which has recently shown to be involved in temperature sensation in humans $(60,62,88)$. Accordingly, these observations have provided strong evidence for the role of the posterior ventral medial nucleus (VMpo) of the macaque thalamus as a potentially pure temperature-specific thalamic rely site for temperature (cold) sensation in this mammal (60).

This recent evidence on the role of the primate's posterior ventral medial nucleus (VMpo) of the thalamus in thermosensory integration has opened to a better understanding of the neuroanatomy of the temperature-specific sites of thalamic somatosensory integration. However, these findings have also questioned the role of previously reported temperature-dependent activity in the ventral posterior lateral (VPL) and medial (VPM) nuclei of the macaque thalamus. In this respect, it deserves mention that as well as cold-sensitive neurons, polymodal nociceptive neurons have also been identified in the ventral posterior medial nucleus (VPM) of the macaque thalamus, and shown to phasically respond to: 1 ) innocuous cooling and mechanical stimuli; 2) noxious heat and mechanical stimuli; 3) innocuous cooling, heat and mechanical stimuli (31). The activation of these polymodal neurons within the ventral posterior medial nucleus (VPM) of the macaque thalamus upon both thermal and mechanical stimulation (31), was recorded in alert behaving animals (as opposed to those studies in which animals were aestheticized). In light of the above, the identification of temperature- and mechano-sensitive polymodal nociceptive neurons in the ventral posterior medial nucleus (VPM) of the macaque thalamus could support the hypothesis for which previously reported temperature-dependent activity in the ventral posterior lateral (VPL) and medial (VPM) nuclei of the macaque thalamus might not be temperature-selective. In fact, rather than sub-serving a primary role in temperature sensation, the ventral posterior complex in the primate thalamus could indeed play a complementary, nonspecific role in thermal integration.

The fact that neurons in the ventral posterior medial nucleus (VPM) of the macaque thalamus present convergence from polymodal (i.e. thermal and mechanical) nociceptive afferents could carry implications in the context of cortical processing of somatosensory perceptions. Indeed, it can be speculated that the concurrent activity of multiple thalamic neuronal populations with multimodal properties, could in turn result in broadening the range of potential neural outputs from the thalamus to the cortex. Ultimately, this could underline the ability to characterize and distinguish between different components of somatic perceptions (e.g. exteroceptive vs. interoceptive) (67), as resulting from the combination of different thermal and mechanical stimuli on the skin (31). In support of the hypothesis that multimodal convergence within the thermosensory pathway could underline the ability to discriminate between different thermal stimuli on the skin, psychophysical evidence has repeatedly shown how important cross-modal interactions between thermal and tactile senses are in determining the ability to spatially discriminate thermal sensations across the body (see Psychophysics of temperature sensation - Role of stimulus area paragraph). The low spatial acuity of the thermal sense has been indeed shown to be improved when thermal stimulation of the skin is concurrent to mechanical stimuli (38). The fact that thermo- and mechano-sensitive neurons within the ventral posterior medial nucleus (VPM) of the macaque thalamus present very small mechano receptive fields (as compared to the ones observed for their respective lamina I neurons) (31), might indicate that such touch-related spatial information on skin thermal stimulations could be integrated at different levels of the neuroaxis. Altogether, these observations could highlight the fact that under naturalistic conditions, a potentially more complex (than previously postulated) afferent convergence and processing of somatosensory inputs to the thalamus could occur (258).

While the evidence presented above should be taken into consideration in the context of multimodal thermal processing, it is however important to stress the fact that within the context of pure thermal sensibility, the most recent evidence in primates point to the key role of the macaque's posterior ventral medial nucleus (VMpo) in thalamic thermo-sensory integration (58). The importance of such evidence is further highlighted by the observation that this area is a specific homologue of the posterior part of the human ventral medial thalamus (VMpo) (60), a 
thalamic region which has been proposed as the primary thalamic rely for cold sensation in humans (80).

With regards to the available knowledge on human models of thalamic thermosensory integration, several studies have provided indirect and direct evidence on what thalamic regions could be involved in thermosensory processing. Interestingly, and in line with evidence in primates, it appears that certain human thalamic regions could present neuronal populations responsive to temperature, however in a non-selective and in a non-modality-specific manner; and that other regions could present neuronal populations whose activity is in fact temperature-specific.

Direct evidence for what specific regions of the human thalamus are involved in conveying thermosensory information has been provided by studies endorsing thalamic micro-stimulation during stereotactic surgery in patients with pain and movement disorders $(80,194,195)$, as well as by studies assessing thermo-sensory abnormalities and central pain in stroke patients with constrained thalamic lesions (179). Micro-stimulation of the posterior and inferior ventral caudal nucleus (a portion of the ventral posterior lateral nucleus; VPL) of the human thalamus has been shown to evoke non painful thermal sensations $(194,195)$. Figure 13 shows maps of receptive and projected fields for trajectories in the region of the ventral caudal nucleus in a representative single patient. Also, application of cold stimuli on the related skin receptive fields has been shown to induce activations of single neurons within this thalamic complex (193). Interestingly, as well as to innocuous skin cooling, such cold-sensitive thalamic neurons were also observed to respond to light mechanical stimulation of the skin. From a functional point of view, cold-sensitive third-order neurons in the ventral caudal nucleus of the human thalamus have been shown to respond to cooling stimuli applied to the skin with characteristic bursting discharge (192), similarly to what has observed in first order cold-sensitive afferents. However, as bursting discharge is apparently absent in second order spino-thalamic neurons (88), it is likely that such discharge pattern could be the result of thalamic circuitry rather than being the result of central integration of peripheral inputs (192).

While the evidence reported on the responsiveness of neurons within the ventral caudal nucleus of the human thalamus to skin cooling and mechanical stimulation $(194,195)$ has provided insights on the mechanisms of human thalamic multimodal thermosensory integration, responses to skin cooling alone, evoking pure cold sensations in human subjects, have been recorded in single neurons within the posterior part of the human ventral medial thalamus (VMpo) $(79,80)$. Neurons within this thalamic complex have been reported to respond to small (i.e. $<5^{\circ} \mathrm{C}$ change) cooling steps applied to the corresponding cutaneous region where sensations could be evoked via thalamic (ventral medial) micro stimulation. Also, and similarly to what previously observed for primate lamina I second order neurons (88), these thalamic neurons have been reported to respond dynamically at the onset of cooling, and to then (tonically) stabilizing their response according to the duration of the cooling stimulus (80).

The fact that both the ventral caudal nucleus and the posterior ventral medial nucleus (VMpo) of the human thalamus present thermally-sensitive third order neurons opens to the question of whether these thalamic regions could in fact sub serve different integrative functions within the context of thermal (and somatosensory) integration. The fact that these nuclei receive projections from second-order afferents with different sensory properties, could support this hypothesis.

As part of the ventral posterior complex (i.e. including lateral and medial nuclei), the ventral caudal nucleus of the human thalamus appears to receive inputs from mechano-sensitive neurons responsive to cooling (although these responses are not specifically correlated to temperature), a fact which could indicate that this area might only partially contribute to cold sensation (192). Rather, this region could integrate the tactile component of thermal stimulation of the skin, therefore contributing to multimodal spatial and thermal discrimination. On the other hand, a recent cytoarchitectonic and immunoistiochemical characterization of the human posterior ventral medial nucleus (VMpo) of the thalamus has shown that this area heavily receive projections from lamina I thermally and pain sensitive second order nerve fibers (21). Hence, and similarly to what observed in the macaque thalamus, the posterior ventral medial nucleus (VMpo) of the human thalamus could represent the main rely site for pure cold sensations in the human thalamus $(60,80)$. The fact that this thalamic region has been shown to convey thermo-sensory 
inputs to the insular cortex (a cortical region which has recently shown to be involved in temperature sensation in humans) $(58,60,62,88)$, further support its role as a rely site for pure thermal sensibility within the central thermo-sensory pathway in humans.

In summary, in cats, primates and humans, lamina I temperature-specific neurons project their afferent fibers via the middle lateral funiculus to the posterior part of the ventral medial nucleus of the thalamus (VMpo) $(21,60,64)$. The ventral posterior (lateralis and medialis, VPL and VPM) nuclei appear to receive inputs from mechano-sensitive neurons responsive to cooling (192-195). Both thalamic rely sites receive thermo-sensory information; however, evidence indicates that the posterior part of the ventral medial nucleus of the human thalamus (VMpo) could have developed in a specific rely site for pure thermal sensibility within the human central thermo-sensory pathway, while the ventral posterior (lateralis and medialis, VPL and VPM) nuclei could sub serve a complementary role as a rely-site of thermal-tactile interactions within the somatosensory pathway $(5,88,135)$.

The location, characteristics and function of the human cortical regions, which receive projections from thalamic third order neurons, and which ultimately process conscious skin thermal sensations, will be presented below.

\section{Cortical integration}

The somatosensory cortex has long been considered as the main area involved in the cortical processing of mammalian thermal sensations (45). This view has been supported by the findings of early (as well as more recent) studies in both animal models and humans $(45,143,188,213)$, which had provided evidence for the presence of temperature-responsive neurons in this cortical region. Accordingly, the widely-accepted assumption that the somatosensory cortex would represent the main cortical processing center in thermal sensibility has contributed to the view that temperature discriminability would therefore represent a sub-class of touch perception, retaining only an exteroceptive value in the context of somatosensory function. However, recent neuroanatomical work in primate models (58) along with neuroimaging results from human studies $(61,62,157)$, have repeatedly challenged this view $(67)$. Strong evidence has been indeed provided not only for the fact that other cortical areas than the somatosensory one could be involved in thermal processing, but that in fact these areas could play a more specific role in sub-serving temperature (as well as pain) (263) sensations in humans $(19,62,237,296)$. Amongst these areas, the dorsal margin of the posterior insular cortex has been proposed as the specific area for the cortical processing of both the discriminative (157) and affective $(62,251)$ components of thermal sensations in humans. The fact that this cortical region has been previously shown to receive projection from those third-order thermo-sensory neurons which are located in the posterior ventral medial nucleus (VMpo) of the primate and human thalamus, and which are part of that spino-thalamo temperature pathway representing the main ascending pathway for temperature sensation in both primates (58) and humans (88), has further strengthened the evidence in support of a specific thermo-sensory role for the human posterior insular cortex. Nevertheless, evidence arising from lesion studies observing retained thermosensory function in individuals presenting a disease-induce loss of insular cortices (72), has also questioned the hypothesis $(57,59)$ for which the human dorsal insula would be the exclusive cortical area for the processing of thermal sensibility (72) and of human feelings in general (73). This very recent results have opened to the intriguing hypothesis that, along with alternative cortical areas (e.g. somatosensory and cingulate cortices), sub-cortical regions (e.g. brain stem and hypothalamus) could also contribute to sensory function (amongst which thermal sensibility) in humans (72).

The following paragraphs will present findings on the role of the different cortical regions potentially involved in human thermosensory integration. In light of the comprehensive nature of this review article, the animal and human evidence in support of the long believed selective role of the somatosensory cortex in thermal sensibility will be briefly outlined. Following, recent 
evidence supporting the thermosensory function of the human dorsal insular cortex (and of other cortical regions) will be presented.

The potential role of the somatosensory cortex in human thermal sensibility had been initially inferred based on comparative results from studies in the cat and rat, which had reported the presence of specific thermo-sensitive neurons in this cortical region. Landgren (188) was the first to observe cortical neurons in the cat' somatosensory cortex to respond to peripheral thermal stimulations. As a result of cold stimuli (i.e. cold water) applied to the cat's tongue, these coldspecific neurons were reported to respond phasically, with short latency (15-20 ms) and relatively high frequency (60 - 70 imp's) to tongue's cooling. In line with these results, cortical neurons located in the rat's somatosensory cortex were also observed to selectively respond to heating and cooling of scrotal skin and hind foot (e.g. cold-sensitive neurons showed increase in mean firing upon cooling and suppression upon warming) (143). More recently, further evidence from mammalian models (i.e. mouse) has confirmed the presence of multimodal cold- and touchsensitive cortical neurons in the primary somatosensory cortex (213). Cortical neurons in mice' somatosensory cortex have been indeed shown to respond to paw cooling (as well as to tactile stimuli) and to be essential for behavioral cold discrimination: pharmacological silencing of these multimodal cold-sensitive neurons located in the primary somatosensory cortex was indeed observed to impair thermal discrimination during behavioral thermotaxis in awake mice (213).

Along with supporting evidence from animal studies, the view that the somatosensory cortex would retain temperature-sensitive neurons and would therefore represent the preferential area for the cortical processing of thermal sensations in humans, had also been partly supported by electrophysiological and neuroimaging evidence in humans. Findings from early studies on cortical integration of thermal sensations in humans indicated that skin warming resulted in evoked potentials localized on regions of the human scalp corresponding to the somatosensory cortex (45). Recently, neuroimaging studies have also provided evidence for a strong activation of the primary and secondary somatosensory cortices (along with the posterior cyngulate gyrus and insular cortex) during skin warming (temperature: $\left.41^{\circ} \mathrm{C}\right)(12,251)$.

At this point, while the evidence above would make it tempting to conclude that the human somatosensory cortex plays a key role in thermal sensation, it is important to highlight that for this cortical region to preferentially code skin thermal sensations, the same would have to show activity which is correlated to stimulus intensity (i.e. increasing/decreasing temperature stimuli would be expected to induce correlated changes in neuronal activity in this cortical region). This argument (and related supporting evidence) has represented the basis for the recent dismissal of the view that the somatosensory cortex is the exclusive cortical site for thermal sensations in humans, and for the proposal that the human posterior insular cortex could be in fact the main thermosensory cortex processing cold sensations in the human brain (62).

In a recent study, Craig et al. (62) have indeed provided evidence for the presence of temperature-correlated activity in human posterior insular cortex, and for the concurrent lack of proportional activity in the human somatosensory cortex. In this study, positron emission topography imaging performed during tonic hand cooling (temperature range: 33 to $22{ }^{\circ} \mathrm{C}$ ) in awake humans, indicated that cortical activity in the posterior insular cortex strongly correlated with human discrimination of the intensity of the thermal stimuli (Fig. 14) (62). Remarkably, no correlation was observed between thermal stimuli and activity in the somatosensory cortex, a fact which has strongly supported the new view for which the posterior insular cortex would indeed represent the main cortical site for processing and discriminating human thermals sensations (62) (note: at this point it would appear reasonable to hypothesize that the previously reported temperature-uncorrelated activity in somatosensory cortex during thermal stimulation, could have therefore been the result of thermal-tactile interactions during the application of contact thermal stimuli on the skin of the non-human/human model endorsed). The functional evidence provided by the study of Craig et al. (62), followed previous observations of concurrent activation of the insular and somatosensory cortices during thermal stimulation of the human skin. During hand cooling (temperature: $20^{\circ} \mathrm{C}$ ) and warming (temperature: $40^{\circ} \mathrm{C}$ ) performed in healthy humans 
undergoing positron emission topography imaging, Craig et al. (66) had indeed previously observed concurrent activation of primary and secondary somatosensory cortices, and of the anterior insular cortex (66). Similarly, studies endorsing high resolution functional magnetic resonance imaging of sub-cortical and cortical regions during the application of cooling and warming ramps to the skin of healthy humans, had showed that thermal stimuli induced significant activation of both posterior and anterior insular cortices (along with activation of the ventral posterior lateral nucleus and ventral medial nucleus of the thalamus) $(78,186)$.

In line with neuroimaging work, direct evidence has been recently provided on the activation of cortical regions in the human parasylvian cortex (i.e. lateral sulcus in the parietal lobe; a cortical region that includes the insular cortex), during cold stimulation of the skin. This activity was directly recorded (i.e. evoked potentials) with implanted electrodes in patients with normal cold sensitivity undergoing seizure treatment (129). Figure 15 shows evoked potentials recorded directly from the brain in response to an innocuous cold stimulus applied to the contralateral hand of two female patients who had subdural grids implanted for surgical treatment of medically intractable complex partial seizures. Cold-evoked potential occurred in these patients with mean amplitude of $30 \mu \mathrm{A}$ and a latency of $\sim 800 \mathrm{~ms}$ (129). Based on the above, it was speculated that the recorded temperature-dependent cortical activity in the human parasylvian cortex might have been the result of activity in the near insular cortex. This observation further supported the previously observed cold-dependent activity of what seems to be the main human thermosensory cortex, i.e. the human posterior insular cortex (62). As well as presenting activity which appears to be correlated to the intensity of thermal stimuli, the human posterior insular cortex has also been recently shown to be potentially organized in a somatotopic way (157). By means of functional magnetic resonance imaging, Hua et al. (157) have reported that graded cooling stimuli applied to different body regions (i.e. hand and neck), resulted in selective activations of different foci within the human dorsal insular cortex, which were organized according to an anteroposterior somatotopic pattern (i.e. areas coding thermal stimulation of the neck were anterior to those coding for the hand) (157).

The fact that the human posterior insular cortex could represent a main cortical center for the processing of conscious warm and cold skin thermal sensations is also supported by the clinical observation that lesions of such regions result in complete (296) or in modality-specific (e.g. cold) (19) thermo-anesthesia in humans. For example, in a recent quantitative sensory analysis, warm and cold hypoesthesia was consistently recorded in patients with constrained cortical lesions located in the insular and parietal cortices (296). Interestingly, lesions of the human insular cortex not only induce thermosensory deficits, but also, these are very often accompanied by the development of central pain $(19,296)$. In this respect, the posterior insular cortex has been recently identified to also sub serve a fundamental role in pain processing in humans (263). In light of the potential inhibition that cold exerts on pain (see masking mechanisms operated by lamina I cold-sensitive neurons on heat-pinch-cold sensitive neurons; Spinal Integration section), this observation provides support to the previously proposed hypothesis (62) for which the development of central pain $(19,296)$, as often observed after infarction of the insular cortex, could represent a form of thermosensory deficiency resulting from the sudden lack of temperature-induced inhibition of nociceptive circuits at a cortical level (62). This hypothesis is in line with the view that the ascending thermosensory pathway shares not only anatomical, but also functional properties with the pain pathway (65). The human posterior insular cortex has been proposed as to be part of a spino-thalamo-cortical pathway receiving and processing specific sensory information (amongst which temperature and pain) informing on the homeostatic condition of the body, i.e. interoception $(67,68)$. By processing ascending temperature and pain signals, the posterior insular cortex could ultimately contribute to drive two of the most important behaviors deemed necessary for survival: the thermoregulatory and nociceptive behaviors. The insular cortex is also considered as part of the limbic system, whose efferent projections have a role in the control of important physiological functions (e.g. cardiorespiratory, gastrointestinal, sympathetic) aimed at maintaining body's homeostasis (62). Taken together, the neuroanatomical and functional evidence above point to the role of thermal sensibility as an interoceptive 
experiences necessary for its autonomic and behavioral function in the context of maintaining thermal homeostasis, driving thermal behavior and ensuring survival.

Interestingly, the importance of the human dorsal insular cortex in receiving afferent projections from afferent neurons carrying information on the homeostatic conditions of the body (amongst which temperature, pain, muscular and visceral sensations, hunger, thirst, etc.) (67), seems to be linked to the proposed role of the human anterior insular cortex in contributing to the representation of such bodily feelings and to our awareness of them (57). As a result of these observations, it could be hypothesized that the human insular cortex could therefore represent the main cortical center processing both the discriminative characteristics of a thermal stimulus (i.e. exteroceptive aspect) as well as its hedonic value (i.e. interoceptive aspect) in relation to the current state of the body (e.g. core temperature) $(32,33,68)$ and of the external environment (e.g. ambient temperature), in order to effectively direct behavior $(251,252)$. Such bi-parted central processing of thermal sensation could carry evolutionary implications for evaluation, adaption and survival. The pleasantness of warm gloves in a winter day or the refreshing coldness of diving into the sea in a hot day, are all daily life examples of thermal sensations which, as well as helping defining the properties of our surrounding environment, significantly contribute to our ability to behaviorally regulate our body temperature, as part of our constant pursue for homeostasis.

At this point, it is important to highlight that, while the literature presented above provides strong evidence for the role of human posterior insular cortex in processing skin thermal sensations and for their interoceptive role in driving thermal behavior, evidence arising from lesion studies observing retained thermosensory function in individuals presenting a disease-induce loss of insular cortices $(72)$, has also questioned the hypothesis $(57,59)$ for which the human dorsal insula would be the exclusive cortical area for the processing of thermal sensibility (72) and of human feelings in general (73). In this respect, it has been suggested that other cortical (e.g. cingulate cortices) and sub-cortical regions (e.g. brain stem and hypothalamus), could also contribute to sensory function (amongst which thermal sensibility) in humans (72).

For instance, recent neuroimaging studies on the affective role of thermal stimulation have shown that cortical regions such as the orbifrontal and cingulate cortices seem to contribute to define the affective component (i.e. pleasure/displeasure) of thermal stimulation (251), a fact which further emphasize the interoceptive component of temperature sensation (68). It also deserves mention that results from recent functional magnetic resonance imaging studies in humans have pointed towards the involvement of the anterior cingulate cortex in noxious thermal sensation (186). However, rather than in pure thermal sensation, this cortical region has been proposed as to be involved in processing motivational components related to thermal discrimination $(186,260)$.

Interestingly, it has been recently proposed that the brain stem regions (e.g. nucleus of the tractus solitarious, parabrachial nucleus) and the hypothalamus could actively contribute to produce neural maps of general physiological states (amongst which temperature sensations), which could be then further processed in second order higher areas (amongst which the insular cortex) and enriched in the context of interactions with other sensory-related cortical areas (amongst which the somatosensory cortex), in order to provide us with our remarkable ability of experiencing a large variety of sensations and emotional states $(72,73)$. The fact that detailed animal models (mice) have provided evidence on the importance of brain stem regions such as the nucleus of the tractus solitarious, as well as on the role of hypothalamus, in regulating and maintaining body temperature in mammals (amongst which humans) $(26,218,224)$, could support this intriguing hypothesis and could further strengthen the proposed neuroanatomical and neurophysiological link between thermosensory function and maintenance of thermal homeostasis. Overall, what emerges from the evidence presented so far is that the central mechanisms of temperature sensations are the result of a complex array and interactions between different neural structures. 
In summary, the above presented evidence indicates that, from a neuroanatomical point of view, the specific thermosensory pathway which allows humans to peripherally encode and centrally process skin thermal sensations comprises of: first order thermo-sensitive afferent fibers (i.e. skin thermoreceptors); second order lamina I spino-thalamic neurons projecting to the posterior part of the ventral medial nucleus of the thalamus (VMpo); third order neurons located in posterior part of the ventral medial nucleus of the thalamus (VMpo) projecting to the dorsal insular cortex. Functionally, this neurophysiological pathway sub serves peripheral and central mechanisms underpinning our ability to characterize both the discriminative and affective components of skin thermal sensations in the context of thermal behavior. Interactions with other cortical (e.g. somatosensory and cingulate cortices) and sub cortical regions (e.g. nucleus of the tractus solitarious, parabrachial nucleus and the hypothalamus) likely contribute to enrich the polymodal nature (e.g. thermal-tactile interactions) underpinning human thermo-sensory experiences. The relevance of such sensory interactions will be made evident in the context of the analysis of human hygrosensation (see Neurophysiology of skin wetness perception section).

At this point, one of the outstanding questions which has only recently started to be deciphered, is the one related to what molecular mechanisms underlie temperature transduction in those peripheral skin thermoreceptors which represent the first line of integration of skin thermal sensation. The evidence available on the molecular mechanisms of thermoreception will be presented below.

\section{Thermo-sensitive Transient Receptor Potential (TRP) ion channels}

Unveiling the molecular basis of thermoreception is without doubt one of the most recent and important advances in our understanding of skin thermal sensations. The temperature-dependent generation of action potentials in thermo-sensory neurons has been explained by the presence of specific transmembrane proteins which, by gating changes in ion fluxes across the plasma membrane in a temperature-dependent manner, contribute to generating action potentials at the nerve fiber level in response to temperature stimuli (297). Such protein receptors have been recently identified as to belong to the Transient Receptor Potential (TRP) ion channels family (247).

A transient receptor potential was first observed in 1969 in a mutant fly showing abnormal responses to light (55). In 1989 the transient receptor potential mutant gene was cloned and showed to code for a transmembrane protein with up to eight transmembrane regions (215). This observation led to the conclusion that the transient receptor potential gene could code for membrane ion channels or receptors (117). Since then, much has been done to elucidate the molecular mechanisms that allow membrane ion channels to transduce mechanical (touch), hearing, vision, taste stimuli into electrical signals in the form of action potentials conveying sensory information (117). However, it is only very recently that the molecular mechanisms that allow temperature stimuli to be transduced into action potentials by afferent nerve fibers have been identified and characterized. For reasons of maintaining this review self-contained, and having this area been the object of recent detailed reviews $(117,299)$, the following paragraphs will focus on the most representative studies characterizing the function of temperature-sensitive TRP channels and their potential involvement in human thermal sensations.

Since their discovery (44), temperature-activated TRP ion channels located in the membrane of thermo-sensory neurons have been intensively investigated in a number of species, including fruit flies (114), mice (86) and humans (116). As to date, a number of channels have been identified to gate temperature-transduction within specific temperature ranges: TRPV1 (subfamily Vanilloid, member 1; heat $>42^{\circ} \mathrm{C}$ ); TRPV2 (subfamily Vanilloid, member 2; heat $>52^{\circ} \mathrm{C}$ ); TRPM3 (subfamily Melastatin, member 3; heat $>40^{\circ} \mathrm{C}$ ); TRPV3 (subfamily Vanilloid, member 3; warm $34-38^{\circ} \mathrm{C}$ ); TRPV4 (subfamily Vanilloid, member 4 ; warm $25-43^{\circ} \mathrm{C}$ ); TRPM8 (subfamily Melastatin, member 
8; cool $<25-28^{\circ} \mathrm{C}$ ); TRPA1 (subfamily Ankyrin, member 1 ; cold $<17^{\circ} \mathrm{C}$ ). Such channels can without doubts be defined as the putative temperature receptors $(299,301)$.

Contrary to other TRP ion channels, for which temperature acts only a modulator of their activity, a distinctive feature of temperature-activated TRP ion channels is that these channels can be selectively activated by temperature alone. Furthermore, these appear to be specialized per range of temperatures, covering the entire noxious/non-noxious temperature range of mammalian thermo-sensation (242). Another distinctive feature of some temperature-activated TRP ion channels is that, as well as responding to temperature, some of these channels are activated by specific chemicals (e.g. menthol, capsaicin) which mimic the effects of a physical temperature stimulus. For example, by binding to heat-sensitive TRPV1 channels, capsaicin (an alkaloid found in hot chili peppers) depolarizes the sensory neurons where TRPV1 channels are expressed, and mediates the firing of action potentials which lead to the characteristic hot sensations induced by e.g. spicy food $(44,77)$. Similarly, menthol (an organic compound obtained from mint) binds to cold-sensitive TRPM8 channels and induce cold sensations $(86,208,243)$. All in all, the fact that these channels present chemical sensibility, i.e. chemesthesis, provides evidence for their ability to be activated by ligand.

Due to challenges of examining neuro-molecular function in humans in vivo, most of our current knowledge on the molecular properties of thermo-sensitive TRP ion channels arises primarily from research based on animal models. Hence, the following paragraphs will review findings from the most representative studies which have contributed to the discovery and characterization of temperature-activated TRP ion channels in non-mammalian and mammalian-species. Where allowed, analogies and comparative observation with humans will be performed.

Initially, a brief account of the structure and function of typical TRP ion channels will be outlined. Following, an overview of the characteristics of the channels involved in the transduction of both non-noxious and noxious cold and warm temperatures, will be presented.

\section{Structure and temperature-dependent gating mechanisms of TRP ion channels}

It has been established that the presence of TRP ion channels on the cellular membrane of sensory neurons allows the regulation of ion flux across the plasma membrane in a temperaturedependent manner, a fact which ultimately contributes to the generation of action potentials in neurons responding to temperature stimuli (297). Temperature-dependent firing in thermosensory neurons seems therefore to be mediated by temperature-dependent gating and selective permeability of those TRP ion channels expressed on the plasma membrane of these sensory neurons (299). While the key role of TRP ion channels in gating temperature at a molecular level is now widely accepted, the knowledge on the structural characteristics and functional mechanisms that allow thermo-sensitive TRP ion channels to gate ion fluxes in a temperaturedependent manner, is still far from being comprehensive (117). Nevertheless, recent studies have provided initial insights on these mechanisms.

The structure of a heat sensitive TRPV1 channel has been very recently revealed by Liao et al. (197) and these findings have just opened to a better understanding of the tridimensional structure of these transmembrane protein receptors (197). Figure 16 shows the tridimensional structure of the TRPV1 ion channel (determined by electron cryomicroscopy). A typical TRP channel contains six regions spanning across the cell membrane with a pore-forming reentrant loop between the fifth (S5) and the sixth (S6). All TRPV family members contain 3 to 4 ankyrin repeats in their $\mathrm{N}$-terminal cytoplasmic tail. TRPM subfamily members contain a 25 -amino acid motif called the TRP domain C-terminal to S6 (this includes the so-called TRP box), as well as a proline-rich region called C-terminal to the TRP domain (also known as TRP box 2) (197). Interestingly, the C-terminal domain seems to determine the thermo-sensitivity of TRP channels (28). In a study in which chimeric channels TRPV1 and TRPM8 presented swapped C-domains 
(i.e. the TRPV1 channel presented the TRPM8 C-domain and vice versa), the heat-sensitive TRPV1 channel was shown to respond to cooling, while the cold-sensitive TRPM8 ion channels responded to heating (28). However, despite the coupling between the C-terminal domain and temperature was maintained, chimeric channels' responses to temperature occurred with a lower efficiency (28). Altogether, these observation provided evidence for the fact the C-terminal domain could play a role in the channel's gating behavior, and in the regulatory process underpinning the conversion of free energy (i.e. temperature) into mechanical work (i.e. channel opening) (28).

With regards to the functional mechanisms underpinning the ability of thermo-sensitive TRP ion channels to convert temperature into electrical signals in the form of action potentials in sensory neurons, it has been proposed that temperature transduction in thermo-sensitive TRP ion channels is tightly based on mechanisms of voltage-dependent gating (298). These channels' thermosensitivity has been shown to be modulated by transmembrane voltage, and changes in temperature seems to result in graded shifts in the voltage dependence of channel activation (298). Based on these observations, it has been proposed that thermo-sensory TRP ion channels can be considered as voltage-dependent channels: membrane depolarization activates the channel while repolarization closes the channel. Temperature has an effect on shifting the voltage dependence of activation and the channels' specific thermosensitivity arises from the difference in activation energies associated with voltage-dependent opening and closing (298). The kinetic analysis of TRP channels gating at different temperatures has indicated that temperature sensitivity in TRPM8 and TRPV1 channels arise from a tenfold difference in the activation energies associated with voltage-dependent opening and closing (298). As a result of this observation, an index of TRP channels thermosensitivity and temperature-dependent activity has been derived and defined as $\mathrm{Q}_{10}$ (dimensionless). This value represents the difference in the current amplitudes generated by the same TRP ion channel when this is exposed to two different temperatures $10^{\circ} \mathrm{C}$ apart. The amplitude change resulting from the two temperatures is currently used as an indicator to quantify ion channels thermosensitivity (299). Chemical agonists such as menthol (activating TRPM8) and capsaicin (activating TRPV1) function as gating modifiers, shifting activation curves towards physiological membrane potentials, ultimately mimicking thermal responses (298).

It deserves mention that, despite the evidence available appears to support the view that temperature transduction in TRP channels is based on voltage-dependent gating mechanisms (299), a recent work by Clapham and Miller (52) has proposed the hypothesis for which TRP channel gating could be primarily operated via conformational changes in transmembrane proteins, which could be dependent on the heat capacity of these channels when in open or closed conditions. According to this recently proposed thermodynamic framework, all thermosensory TRP ion channels could be both cold and hot sensing, with differences in their respective activity resulting from differences in the heat capacity between the channels' open and closed states (52). Claphan and Miller (52) propose that temperature-dependent changes in the channel open/close state depends upon protein conformational changes which can be described as variations from a constant conformational equilibrium point $\mathrm{K}$. This temperature-dependent conformational equilibrium is the same for both and cold channels and it is described by a nonmonotonic function (i.e. U-shaped) (52). Based on this function, it is speculated that both heatand cold-sensitive TRP channels could be activated by both hot and cold temperatures. However, the fact that a channel's full range of temperature activation would be only partly visible at temperatures between 10 and $50^{\circ} \mathrm{C}$, along with the inability to access the full temperaturedependent range of activation experimentally, would result in attributing to each channel a oneside only (e.g. cold or warm) temperature-specificity, despite their overall thermal sensitivity. While this theoretical thermodynamic model is of interest, until the same is tested under strict experimental conditions and evidence is provided on the concurrent activation of the same TRP channels at both hot and cold temperatures, our understanding of the functional mechanisms underpinning TRP channels thermo-sensitivity remain limited and partially speculative. 
At this point, and while inviting the reader to keep in mind that our understanding of the functional properties of thermo-sensitive TRP channels appears to be regularly challenged by new discoveries in this field, the following paragraphs will outline the currently proposed categorization of temperature-sensitive TRP channels. Transduction mechanism for noxious heat, non-noxious warmth, non-noxious cold and noxious cold will be presented below.

\section{Heat transduction}

As a transducer of painful hot stimuli in vivo $\left(>40^{\circ} \mathrm{C}\right)$, the capsaicin receptor vanilloid receptor 1 (subsequently renamed TRPV1) was firstly identified as being part of a subfamily of TRP channels in 1997 by Caterina et al. (44). Such identification followed the long-standing observation that hot spicy food (containing capsaicin) could induce burning pain sensation, which are known to be mediated by specific nociceptors. As these hot burning sensations can be also evoked by physical stimuli such as hot temperatures, the possibility that a specific molecular receptor, sensitive to both noxious chemical and physical (i.e. heat) stimuli, would sub serve transduction mechanisms for heat sensitivity in peripheral nocicereceptive fibers, led to the discovery of TRPV1 (44).

The capsaicin receptor TRPV1 was firstly identified as an integral membrane protein homologous to store-operated calcium channels (44). This channel was found to be exclusively expressed on small diameter sensory neurons and to be activated by hot temperature within the noxious range $\left(>40^{\circ} \mathrm{C}\right)$, as well as by capsaicin, with a response profile very similar to that of temperature (Fig. 17) (44). Panel $B$ of figure 17 shows an example of how exposure to heat and capsaicin of Xenopus (i.e. clawed frog) oocytes cells transiently transfected with TRPV1, generates inward currents with very similar response profiles. A successive study from Caterina et al. (42) showed that, by using a variety of cellular and behavioral assays, disruption of the TRPV1 gene in mice could eliminate capsaicin sensitivity, demonstrating that TRPV1 could be an essential mediator of the actions of these compounds in vivo (42). It was also show that the occurrence of heat-evoked currents of the moderate threshold class (i.e. $>43^{\circ} \mathrm{C}$ ) was reduced in cultured sensory neurons obtained from TRPV1-deficient mice (42). Sensory neurons and first order afferent fibers from TRPV1-deficient mice showed a marked reduction in proton $(\mathrm{pH} 5)$ sensitivity in vitro, supporting the notion that TRPV1 could also contribute to acid-evoked nociception (42). Finally, TRPV1deficient mice were shown to exhibit marked (although incomplete) deficits in their behavioral responses to noxious heat stimuli, a fact which provided evidence for the notion that the TRPV1 ion channels could be an essential transducer for heat nociception in mammals (42).

Successive studies with mutant mice lacking TRPV1 $(115,247)$, as well as pharmacological studies in humans (116), have strengthened the evidence for the role of TRPV1 in transducing noxious heat at a molecular level, and for its involvement in conveying conscious hot sensations which carry behavioral as well as autonomic implications for the mammalian thermoregulatory behavior. For example, in a recent study, Pogorzala et al. (247) have showed that selective ablation of TRPV1-expressing neurons resulted in mice lacking aversion to noxious hot temperatures $\left(>40^{\circ} \mathrm{C}\right)$. As part of the same study, selective ablation of TRPV1 or TRPM8 channels resulted in insensitivity to heat and cold respectively, along with a shift in mice preferred temperatures (247). It was therefore inferred that both TRPV1 and TRPM8 could mediate aversion for noxious temperature and that their concurrent activity could also contribute to define the range of preferred cool and warm temperatures which are deemed behaviorally and autonomically optimal in maintaining thermal homeostasis (247). In support of this hypothesis, ablation of both TRPV1 and TRPM8 channels appears to result in the absence of a preferred temperature range in mice. The relevance of these findings is in highlighting the fact that afferent thermo-sensory activity gated by a channel such as TRPV1 (and TRPM8) could be critical in providing conscious feedback on noxious temperatures, as well as in determining a preferred temperature range within the non-noxious range. 
As well as for its role in transducing noxious heat stimuli, TRPV1 has been suggested to play a role in modulating autonomic thermoregulatory responses. Selectively blocking TRPV1 channels via pharmacological manipulation operated systemically, has been shown to result in sustained increases in body core temperature (i.e. hyperthermia) in rodents, dogs and humans $(115,116)$. Such hyperthermic responses were observed to result from a lack of TRPV1-induced skin vasoconstriction and an increased thermogenesis, thus indicating a potential role for such heat sensitive channel in contributing to thermal homeostasis and body temperature regulation in mammals, including humans $(115,116)$. However, it has been recently proposed that the involvement of TRPV1 in temperature regulation might not be directly mediated by temperature, but in fact, this could be driven by non-thermal factors (e.g. changes in pH levels, inorganic cations) (255).

At this point, despite the evidence for the primary role of the TRPV1 ion channel in heat transduction $(42,44)$, it has been speculated that TRPV1 alone would not necessarily account for the whole spectrum of heat nociception $(77,247)$. The existence of residual heat-defense responses in TRPV1-deficient mice, as observed in some studies (42), argues on whether heat nociception could involve transduction mechanisms other than those sub-served by the TRPV1 ion channel alone. While mutant mice lacking TRPV1 ion channels only still exhibit some level of heat avoidance, ablation of the sensory neurons in which the same channels are expressed appears to suppress the same heat-defense behavior, an observation which would support the hypothesis that alternative channels, as expressed on the same neurons, could contribute to temperature nociception (247).

In this respect, as homologous of TRPV1, the vanilloid receptor like 2 (subsequently renamed TRPV2) was firstly identified as a potential candidate for higher threshold heat sensitivity $\left(>52^{\circ} \mathrm{C}\right)$ (43). This ion channel was shown to be activated by heat stimuli above the known temperature range for TRPV1, but not by chemicals such as capsaicin (43). Also, this channel was shown to be expressed on first order sensory neurons with medium to large diameters, resembling the previously described heat and mechano sensitive nociceptive C-fibers (heat threshold: $\sim 53^{\circ} \mathrm{C}$ ) (134). Despite these initial results, the role of such molecular receptor in heat transduction has been however ruled out, when genetic ablation of TRPV2-expressing sensory neurons was shown not to impair heat sensitivity in behaving mice (239). Recently, TRPV2 knock-out mice has been indeed shown to present normal behavioral responses to both noxious heat $\left(>52^{\circ} \mathrm{C}\right)$ and to punctate mechanical stimuli (239). In line with these behavioral findings, electrophysiological recordings from skin afferents showed that $\mathrm{C}$-fiber responses to heat, as well as $\mathrm{C}$ - and $\mathrm{A}$-fibers responses to noxious mechanical stimuli, were unimpaired in the absence of TRPV2 channels. Overall, these findings strongly indicated that TRPV2 might not be essential for heat and mechanical nociception in mice (239).

In contrast with what observed for the TRPV2 ion channel, an alternative molecular transducer of noxious heat has been recently identified in the TRPM3 ion channel (300), a member of the Melastatin subfamily of TRP channels with limited homology to the heat-sensitive TRPV channels. TRPM3 has been shown to be expressed in a large subset of small-diameter sensory neurons from mice dorsal root and trigeminal ganglia and to be steeply activated by heating $\left(\sim 40^{\circ} \mathrm{C}\right)(300)$. From a behavioral standpoint, TRPM3-deficient mice present reduced noxious heat-defense responses. Autonomically, the same animals a limited ability to develop inflammatory heat hyperalgesia. Altogether, these observations have pointed towards the role of TRPM3 as a thermosensitive nociceptive channel implicated in the detection of noxious heat (300). Although TRPM3 appeared to share only limited sequence homology with a more established heat nociceptor such is TRPV1, the work of Vriens et al. (300) has provided evidence for surprising functional similarities between these heat-activated channels. Both ion channels are indeed heat-activated calcium-permeable channels (although TRPM3 appears to have a higher heat threshold than TRPV1, i.e. $\sim 50^{\circ} \mathrm{C}$ ) (142); both channels are expressed in a large proportion of small-diameter sensory neurons; both channels appear to contribute to defensive behaviors to noxious heat and chemical agents; both channels significantly contribute to inflammation-induced heat hyperalgesia (300). Interestingly, the characterization of the sensory neurons in which 
TRPM3 is expressed revealed that some of these presented selective expression of TRPM3 channels only, while others presented co-expression of TRPM3 and TRPV1. The fact that certain sensory neurons could co-express TRPM3 and TRPV1 ion channels, along with fact that such channels could both transduce heat stimuli, would therefore explain why previous studies have observed that selective elimination of TRPV1 channels only results in a limited loss of heat nociceptive behaviors in mice $(42,44)$, unless the neurons expressing TRPV1 (and potentially TRPM3) are ablated (247).

In summary, animal models have provided behavioral and genetic evidence for the role of two main ion channels primarily involved in heat transduction, namely TRPV1 and TRPM3. TRPV1 seems to mediate heat nociception at lower temperatures $\left(>40^{\circ} \mathrm{C}\right)$ while TRPM3 presents a higher temperature thresholds $\left(>50^{\circ} \mathrm{C}\right)$. Both channels appear to be expressed in the mammalian somatosensory system in small diameter, dorsal root ganglion unmyelinated nociceptive neurons; and to modulate behavioral and (potentially) autonomic heat-defense responses.

Aside to the noxious (heat) range, different ion channels have been identified to mediate mammalian temperature transduction within the non-noxious warm range. The properties of these channels will be presented below.

\section{Warmth transduction}

As molecular transducers of warmth (temperature range: 25 to $43^{\circ} \mathrm{C}$ ), TRPV3 and TRPV4 ion channels have been suggested to potential candidate for mammalian non-noxious warm detection $(50,51,131,190,244,269,302,308)$.

The potential role of a specific set of TRP channels in non-noxious warm transduction was firstly proposed by Peier et al. (244), who cloned TRPV3 channels and found that TRPV3 was a cation permeable channel activated by warm to hot temperatures (activation threshold of 33 to $35^{\circ} \mathrm{C}$, with ongoing activity at temperatures up to $42^{\circ} \mathrm{C}$ ). Interestingly, and as opposed to other thermosensitive TRP channels, the TRPV3 ion channel was found to be expressed in the keratocynes of adult rat skin tissue, rather than in dorsal root ganglion neurons. In parallel with the work of Peier et al. (244), both Smith et al. and Xu et al. $(269,308)$ identified TRPV3 as a capsaicin insensitive, vanilloid receptor-like protein, responding to temperatures between 25 and $37^{\circ} \mathrm{C}$ and also to heat $\left(>40^{\circ} \mathrm{C}\right)$. Xu et al. (308) found that TRPV3 channels expressed in ovary hamster cells increased their activation with raising temperatures from 23 to $36^{\circ} \mathrm{C}$. Similarly, Smith (269) found that TRPV3 responded to increasing temperatures with a threshold at $\sim 39^{\circ} \mathrm{C}$, and ongoing activity at temperatures up to 48 to $50^{\circ} \mathrm{C}$.

Along with TRPV3, the TRPV4 ion channel was identified to be also involved in warm temperatures transduction (131). The TRPV4 ion channel was reported to be expressed in the preoptic area of the rat hypothalamus as well as in keratocynes, and to be activated by warmth (131). This channel was shown to be particularly active during temperature ramps in the range of 27 to $45^{\circ} \mathrm{C}$, and to present a temperature threshold at $\sim 27^{\circ} \mathrm{C}$, a temperature which appeared much lower than the one recorded during activation of TRPV1 (i.e. $\sim 42^{\circ} \mathrm{C}$ ). Temperaturedependent significant increases in intracellular calcium during activation of TRPV4 confirmed the calcium-gating activity of this warm sensitive channel. Further evidence in support of the role of TRPV4 as additional warm sensitive channel was provided by the work performed by Watanabe et al. (302) and Chung et al. (50,51), who showed that TRPV4 could represent a functional temperature-sensing channel expressed in keratocynes, likely involved in temperature-dependent (range: $25-43^{\circ} \mathrm{C}$ ) calcium signaling. In light of the above, both TRPV3 and TRPV4 appeared therefore to selectively mediate membrane depolarization in sensory neurons upon non-noxious warming. Interestingly, a striking and shared feature of these ion channels is that these appear to be primarily located in skin keratocynes rather than in dorsal root ganglions $(50,51,131,190$, $244,269,302,308)$. 
In this respect, the potential mechanism through which keratocynes could signal warmth to nearby skin free nerve endings in order to activate warm sensitive C-warm fibers located in the dorsal root ganglions, has been suggested to be dependent upon heat-activated secretion of adenosine triphosphate (ATP) (204). ATP appears indeed to be a messenger molecule for TRPV3-mediated warmth transduction in the skin (204). In a recent study, Mandadi et al. (204) have reported that a typical TRP channel-gated heating-dependent increase in cytosolic calciumconcentration in TRPV1 deficient dorsal root ganglion neurons occurred only when neurons were co-cultured with keratinocytes. In line with this, it was also shown that ATP release in keratinocytes was compromised in TRPV3-deficient mice. This recently discovered ATPdependent mechanism for warmth transduction in keratocynes, as operated by TRPV3 ion channels (204), has highlighted the possibility that the skin in itself could represent the first biological transducer of non-noxious warm temperatures $(50,51)$.

The physiological significance of the thermo-sensory properties of mammalian skin could rely on the fact that, in an optimal thermo-sensory system, it would beneficial to have warmth detectors located away from any other potential source of warmth within the system, so that these detectors could efficiently respond to external stimuli (e.g. changes in environmental temperature) with limited thermal bias from the system itself. In case of living mammals (e.g. mice and humans), a potential source of warmth within the thermo-sensory system is represented by body core temperature. Core temperature of mammals such as mice and humans, is tightly regulated at $-37^{\circ} \mathrm{C}$, an absolute temperature which is above the activation threshold of TRPV3 channels (i.e. $\sim 34^{\circ} \mathrm{C}$ ). In light of this, it is therefore reasonable to hypothesize that, the expression of TRPV3 channels in tissues (e.g. skin) whose temperatures are similar (if not lower) to that of the channel's activation threshold, would ensure that these channels are not constantly triggered by normal core temperature, but rather, by changes in skin temperature above the channel's activation threshold. In this respect, the fact that the temperature of non-glabrous skin is often regulated at $\sim 34^{\circ} \mathrm{C}$ under normal temperate conditions, could provide support to the fact that this biological tissue could be ideal for the expression of warmth sensitive molecular transducers. In line with this view, the presence of heat sensitive $\left(>42^{\circ} \mathrm{C}\right)$ channels expressed on dorsal root ganglions neurons (i.e. closer to deep tissue in the body) further supports the potential physiological significance of the expression of TRPV3 (and potentially TRPV4) warmth sensitive ion channels on skin keratocynes (244).

Along with the genetic and physiological evidence available on the warmth sensitivity of these channels, recent studies have also confirmed the behavioral importance of TRPV3 and TRPV4 channels in mediating non-noxious warm detection $(190,216)$. Behavioral assays of thermo-taxis in TRPV3 and TRPV4-deficient mice has indeed indicated that disruption of these channels could impair animals' thermal sensibility $(190,216)$. Warmth-activated mouse keratocynes, responding to warmth (temperature threshold: $-33^{\circ} \mathrm{C}$ ) and camphor (i.e. a natural compound that modulates warm sensations in humans) have been shown to be important for mammalian thermal sensation, as TRPV3-null mice present selective deficits in thermo-sensory discrimination in response to non-noxious warmth and noxious heat (216). Similar behavioral results were observed with mice lacking TRPV4 ion channels (190). Thermal selection behavior appears altered in TRPV4-lacking mice, as these animals tend to spend an unusual prolonged time at temperatures higher than those preferred by control mice, while also showing reduced heat-defense responses to moderately hot stimuli (190). Interestingly, and contrary to what observed for TRPV1 channels (115), inactivation of TRPV4 ion channels does not appear to have any modulatory effect on body temperature regulation (190).

Despite the genetic and behavioral evidence outlined above, the potential primary role of mammalian TRPV3 and TRPV4 ion channels in selectively transducing warm temperature stimuli has been recently challenged, due to the potential influence that animals' genetic background could have played on the previously reported impaired warmth detection in mutant mice lacking TRPV3 and TRPV4 ion channels (158). In a recent study, Huang et al. (158) have reported that genetic deletion of the TRPV3 alone or in combination with the TRPV4 ion channel, had little effect on mouse warmth detection. These results were interpreted as to provide evidence for the possibility that the background strain of mice used as animal models could be a key modifier of 
thermal sensation (158). TRPV3 and TRPV4 were indeed shown to make a limited and straindependent contributions to innocuous warm temperature perception, a fact which led to the hypothesis that other significant mechanisms for warmth detection could exists (158). In this respect, members of the 2 pore domain potassium channels $(\mathrm{K}+)$, specifically TRAAK and TREEK-1 and 2, have been shown to present warmth sensitivity in the range of 20 to $40^{\circ} \mathrm{C}$, a fact which has opened to the hypothesis that these channels could also contribute to warmth (and potentially cold) transduction $(170,228)$. However, rather than in primarily driving temperaturedependent action potentials, these channels contribution to temperature gating appears to be related to modulating sensory neurons' resting membrane excitability (170). Along with the voltage gated sodium channels, these potassium channels are indeed known to be important regulators of sensory neurons' resting membrane potentials, and to influence the voltage threshold for the generation of the action potential in sensory neuron throughout the central and peripheral nervous system $(93,250)$.

Recently, Kang et al. (170) have proposed that with an increase in temperature, TREK-1 and TREK-2 would tend to suppress excitation produced by other TRP channels, likely as a way to prevent over-stimulation of TRP ion channels during warmth/heat exposure. On the contrary, colder temperatures appear to reduce TREK-1, TREK-2 and TRAAK current and increase membrane excitability. In light of this, it has been proposed that TRP and TREK ion channels could therefore interact in transducing thermal signals into action potential via an acute or steady modulation of sensory neurons excitability (170). This hypothesis could be supported by some recent observations provided by Noel et al. (228), who have reported that temperature-sensitive potassium channels of the TREK/TRAAK family, could also be implicated in temperature transduction, by contributing to the temperature thresholds and ranges in which temperaturedependent activation of thermo-sensory TRP channels occurs (228). For example, deletion of these channels has been shown to unsilence heat sensitive neurons and to reduce their firing threshold by $3-5^{\circ} \mathrm{C}(228)$. Also, their deletion was shown to result in an increase of the firing properties of $\mathrm{C}$-fibers within a temperature range of 35 to $46^{\circ} \mathrm{C}$ in knockout mice (228). These potassium channels seem therefore to control the sensitivity of thermo-sensitive TRP channels, as their removal appeared to unsilenced nociceptors which are usually activated at much lower (on the cold side) and higher (heat side) temperatures (228). The fact that deletion of TREK-1 and TRAAK channels primarily results in lower temperature thresholds and increased firing activity in nociceptive (e.g. heat) neurons, could be consistent with the idea that temperaturesensitive TRAAK or TREK-1 channels modulates resting membrane potentials in sensory neurons, thus influencing the temperature threshold of the specific TRP (or TRP-like) channels sub serving primary responses to initial changes in temperature (170). Further evidence is however needed in order to strengthen these hypotheses on the interaction between thermosensory specific (e.g. TRP) and non-specific (e.g. $\mathrm{K}+$ ) ion channels in temperature transduction. In the context of human pathophysiology, increasing the knowledge on the function and interaction between TRP and $\mathrm{K}+$ ion channels is of clinical significance for the study of those demyelinating diseases, amongst which multiple sclerosis, which present changes in membrane excitability and potassium channels function within the central nervous system (93), along with characteristics mechanisms of heat sensitivity resulting in temporary worsening of clinical symptoms $(82,281)$.

In summary, the above presented evidence indicate that TRPV 3 and TRPV4 channels, as expressed in skin keratocynes, along with 2 pore domain potassium channels (contributing to the modulation of the membrane potential of sensory neurons), could be involved in the molecular transduction of non-noxious warm temperatures in mammals. However, as evidence has arisen for the fact that warmth transduction in TRPV3 and TRPV4 channels could be limited and straindependent, our knowledge on the molecular mechanisms for innocuous warm temperature perception remain fragmentary. 


\section{Non-Noxious Cold transduction}

The first cold-sensitive TRP channel was identified by McKemy et al. (208) and Peier et al. (243). McKemy et al. (208) showed that the CMR1 (Cold-Menthol-Receptor 1) ion channel in the rat (whose human orthologous is the TRPM8 ion channel), a channel expressed on small diameter neurons in trigeminal and dorsal root ganglia, was responsible for mediating cold transduction upon cooling (temperature range: $8-28^{\circ} \mathrm{C}$ ) and was also activated by agents such as menthol (a compound previously known for inducing cool sensations upon application to the skin). Rat trigeminal neurons were shown to increase their excitation upon cooling and menthol application with a concurrent increase in intracellular free calcium gated by the temperature-dependent activity in this cold-sensitive channel. Warm stimuli decreased excitation in the same neurons (208). Similarly, Peier et al. (243) identified the mouse TRPM8 ion channel as to be a specific cold and menthol receptor. Mouse cells overexpressing TRPM8 were shown to respond to cold with increases in intracellular calcium upon cooling in the range of 25 to $15^{\circ} \mathrm{C}(243)$. Specifically, a temperature threshold for the increase in intracellular calcium in cells maintained at an initial temperature of $34^{\circ} \mathrm{C}$, was found at $\sim 23^{\circ} \mathrm{C}$. Similarly to what observed by McKemy et al. (208), such receptor was shown to be expressed in a sub-population of small diameter thermoreceptive and nociceptive neurons (distinct from heat and pain sensing neurons) in the dorsal root ganglia and to be also activated by menthol (243). Recently, the importance of TRPM8 as a cold receptor has been elegantly confirmed molecularly, neurophysiologically and behaviorally in a mouse model by Bautista et al. (11). TRPM8-deficient mice were shown to present cutaneous C-nerve fibers which lacked responses to menthol and cooling, and as a result, TRPM8-deficient mice lacked behavioral response to cold in the temperature range of 25 to $10^{\circ} \mathrm{C}(11)$. Such findings are summarized in Figure 18, which shows results from analysis of cold-evoked responses in cultured sensory neurons and intact sensory nerve fibers from TRPM8-deficient mice, as well as behavioral discriminatory ability between cold and warm surfaces, and responses to evaporative cooling, in TRPM8 mutant mice.

Further evidence for the role of TRPM8 as a potentially universal mammalian non-noxious cold receptor has been provided by studies using behaving rodents lacking TRPM8 $(54,86,213,247)$, which have repeatedly shown that animals lacking TRPM8 present an impaired non-noxious cold detection. For example, when exposed to temperatures ranging between noxious cold to noxious heat, TRPM8-deficient mice consistently spend a significantly longer time at colder temperatures than their normal control, which on the contrary, show preference for warmer temperatures. Such altered behavior can be interpreted as the result of a reduction in cold sensitivity in TRPM8deficient mice, which in turns impairs the animal's ability to recognize the normally "unpleasant" colder temperatures $(54,86)$. Overall, the relevance of such findings lay on the fact that the mouse TRPM8 ion channel is known to be 93\% identical to that of human gene (243), thus indicating the potential for the same channel to sub serve cold-transduction, and therefore cold thermal sensations, also in humans.

Despite in the past 13 years consistent evidence has been provided for the importance of TRPM8 as molecular mediator of cold sensations in a number of mammalian $(209,210)$ and nonmammalian (114) animal models, it is not until recently that the expression of this channel on those specific first order thermo-sensory afferents, which have been repeatedly shown to respond to skin cooling and to convey temperature-dependent sensory inputs within the spino-thalamocortical tract in mammals, has been thoroughly characterized (85). Dhaka et al. (85) have shown that in mice, TRPM8 appears to be expressed on a specific population of first order neurons, whose body are located in the dorsal root ganglion, and whose projections terminate in the skin at the level of the stratum spinosum and granulosum (i.e. epidermis) as free nerve endings. These TRPM8-expressing free nerve endings were shown to respond to cold and menthol and not to express any nocicereceptive marker (85), thus indicating this as specific labelled line for nonnoxious cold detection. These observations appear in line with previous results on the electrophysiological properties of first order cold-sensitive thermoreceptors (135).

Finally, a comprehensive overview on the physiological and behavioral role of TRPM8 as primary mammalian non-noxious cold receptor, has been very recently reported by Milenkovic (213) as 
part of a newly characterized and specific somatosensory circuit for cooling perception in mice. The characterization of this somatosensory circuit for cooling perception showed that the transduction of non-noxious cooling (temperature range: 32 to $12^{\circ} \mathrm{C}$ ) appears to be specifically mediated by temperature-dependent gating of TRPM8 channels expressed on first order C-nerve afferents conveying cooling information to the mouse primary somatosensory cortex, ultimately contributing to the animal's cold-driven behavior. The neurophysiological importance of TRPM8 was shown when disruption of this channel resulted in an impaired response to cooling of first order and cortical neurons within this specific thermo-sensory pathway for cooling perception (213).

At this point, although these results have supported the view that TRPM8 could represent a selective molecular transducer for non-noxious cold temperatures, recent results on the responsiveness of TRPM8-expressing cold-sensitive first order neurons to noxious cold temperatures have opened to the hypothesis that TRPM8 could sub serve the transduction of multiple and varied cold-related signals in vivo (e.g. from innocuous to painful cold temperatures, from cold allodynia to cold analgesia) (183). Ablation of TRPM8-expressing neurons has been indeed shown to induce selective loss of specific sensory features, such as cold and cold pain sensitivity in mice models (183). Specifically, selectively ablating TRPM8-expressing neurons in vivo resulted in mice: 1 ) being insensitive to both innocuous and noxious cold; 2) showing attenuated cold hypersensitivity after injury; 3 ) displaying a loss of cold analgesia after injury; 4) showing normal heat sensation, mechanosensation, and proprioception $(182,183)$. Altogether, such findings have led to the recent view that TRPM8-expressing neurons could therefore represent a broad labelled line for cold detection in mammals and that, due to its role in mediating noxious cold temperatures, the TRPM8 ion channel could in fact have a general role in cold detection over a wide range of temperatures (down to $\left.5^{\circ} \mathrm{C}\right)(182,183)$.

Interestingly, as to highlight the autonomic importance of cold detection within the context of maintaining thermal homeostasis, TRPM8 has been shown to be critical, not only for its role in mammalian cold detection, but also, for its role in modulating thermoregulatory responses for cold defense (i.e. vasoconstriction and thermogenesis) (3). In a mouse model, pharmacological blockade of the cold receptor TRPM8 during environmental exposure to cold was indeed shown to induce a decrease in deep body temperature as a result of the inactivation of autonomic colddefense mechanisms (i.e. vasoconstriction and brown adipose tissue thermogenesis) (3). These results have provided the first evidence to date for the involvement of the TRPM8 cold detector (and related cutaneous temperature signals) in the regulation of deep body temperature in a mouse model. It is worth nothing that these results raise the question of to what extent afferents activity in peripheral (e.g. cutaneous) thermo-sensory fibers contribute to the regulation of deep body temperature in mammals $(25,97)$.

In summary, the studies presented above have provided evidence for the role of the mammalian TRPM8 ion channel, as expressed on first order thermo-sensory afferents, in transducing a wide range of non-noxious and noxious cold temperatures. Such finely-tuned cold sensitivity appears to be important: 1) for mammalian cold detection within the conscious thermo-sensory pathway; 2) for thermoregulatory responses for cold defense within the autonomic thermo-regulatory pathway (224).

As it will be presented in the next paragraph, the currently proposed key role of TRPM8 in both noxious and non-noxious cold transduction $(182,183)$ follows what it has been a controversial debate on the potential role of ion channels other than TRPM8 (e.g. TRPA1) (279) in selectively gating noxious cold temperatures (47). The hypothesis for which noxious cold temperature could be selectively gated by ion channels different from those gating innocuous cold (i.e. TRPM8), has been driven by the fact that mutant mice lacking TRPM8 channels only often retain some level of cold avoidance. On the contrary, the ablation of the sensory neurons in which the same TRPM8 channels are expressed, often results in a further suppression of the noxious cold-defense behavior in the same animals (247). As a result, in recent years, alternative ion channels (e.g. TRPA1 and Nav1.8), potentially expressed in the same first order thermo-sensitive neurons as those expressing TRPM8, has been proposed as to be specifically tuned to gate and sub serve 
noxious cold temperature transduction (247). In light of this, an overview of these proposed alternative channels will be presented below.

\section{Noxious Cold transduction}

In light of the inability to induce complete noxious cold avoidance in TRPM8-deficient mice, studies on the molecular mechanisms underlying noxious/non-noxious cold-defense responses had initially supported the view that the role of TRPM8 in noxious cold detection could only be complementary to that of other noxious cold-specific ion channels $(11,86)$. Amongst these alternative ion channels, a prominent candidate for the transduction of cold temperatures within the nociceptive range (i.e. $<17^{\circ} \mathrm{C}$ ) has long been the TRPA1 ion channel (initially known as ANKTM1, subfamily Ankyrin, member 1 ).

The TRPA1 ion channel was firstly identified in mice by Story et al. (279), who found this channel to be particularly active at cold temperatures below $17^{\circ} \mathrm{C}$ and to be expressed on sensory neurons co-expressing capsaicin like receptors (i.e. TRPV1), indicating its potential role in noxious cold and heat transduction, as potentially sub served by those previously identified lamina I polymodal heat-pinch-cold neurons (135) (see Spinal Integration paragraph). TRPA1 was shown to gate rapid increases in intracellular calcium influx upon cooling in the temperature range of 17 to $10^{\circ} \mathrm{C}$, a fact which well matches psychophysical evidence for noxious cold thresholds in humans $(81,128,310)$. Interestingly, exposure to innocuous cooling within that temperature range (i.e. $28-23^{\circ} \mathrm{C}$ ) which is proposed to be primarily sub-served by TRPM8, did not result in any activation of this channels. TRPA1 appeared to be particularly expressed in mice dorsal root ganglion neurons conveying noxious thermal inputs (i.e. polymodal nociceptors) (9). Also, this channels appeared to be activated by pungent compounds such as cinnamon and clove oil, as well as mustard and ginger (9).

The observation of this channel's particular chemesthesis further supported the potential role of TRPA1 as a noxious cold receptor contributing to those burning hot sensations resulting from noxious skin cooling (9). The observation that acute activation of this channel via agonist injection in behaving mice resulted in triggering an immediate nociceptive behavior (i.e. paw licking), strengthened the possibility that this ion channel could transduce both temperature and chemical stimuli which are noxious in nature (9). Indeed, this channel's responsiveness to noxious cold temperatures (279), its expression on nociceptive neurons, and its responses to noxious compounds, supported the view that TRPA1 could be a mammalian channel specifically mediating noxious cold and burning sensations upon noxious cooling and chemical stimulation of the skin $(9,279)$.

Following its initial characterization (279), contrasting results have however started to be reported on the TRPA1 sensitivity to noxious cold temperatures, a fact which has repeatedly challenged the view that this channel could represent a specific molecular transducer of noxious cold (167). For example, Jordt et al. (167) found that $96 \%$ of mustard oil-sensitive neurons from rat trigeminal ganglia, identified as to express TRPA1 channels, were insensitive to noxious cold temperatures $\left(5^{\circ} \mathrm{C}\right)$, while the remaining $4 \%$ showed cold-sensitivity, however via TRPM8-mediated gating mechanisms. Reasons for such contrasting results on the role of TRPA1 in cold transduction had initially remained unclear, until Karashima and Talavera (171) provided evidence in support of the role of TRPA1 as a noxious cold detector in a mouse model, along with potential explanation for the previously ambiguous results on TRPA1 cold sensitivity.

As a result of a comprehensive characterization of TRPA1 properties in vitro and in vivo in a mouse model, Karashima and Talavera (171) have shown this ion channel: 1 ) to be activated by cold temperatures (i.e. $\sim 13^{\circ} \mathrm{C}$ ); 2 ) to present temperature-dependent gating mechanisms similar to those observed in temperature-sensitive TRP channels; 3 ) to show strong activation upon stimulation with mustard oil (in line with previous reporters) (167). Interestingly, the mustard oilinduced activation of this channels appeared to be much larger than that resulting from cold 
stimulation. The fact that cold stimulation could result in smaller amplitudes than mustard oil, could therefore explain why cold-activated TRPA1 currents could have escaped detection in certain expression systems and experimental conditions (167). Indeed, the substantial difference in potency between cold- and mustard oil- induced responses in TRPA1-expressing sensory neurons could have been a confounding factor in indenting the role of this channel in noxious cold transduction (171). Within the same study (171), a specific subset of mice cold-sensitive trigeminal ganglion neurons, expressing TRPA1 channels, were also characterized and shown to present lower cold temperature thresholds $\left(\sim 19^{\circ} \mathrm{C}\right)$ than cold-sensitive neurons expressing TRPM8 channels (threshold at $\sim 25^{\circ} \mathrm{C}$ ) (171). These specific subset of TRPA1-expressing coldsensitive neurons were absent in TRPA1- deficient mice (171). Also, cold plate and tail-flick experiments (i.e. indicators of nociceptive behavior) revealed TRPA1-dependent, cold-induced nociceptive behavior in mice (171). Altogether, these findings provided support for the potential involvement of TRPA1 in mammalian noxious cold transduction.

In line with this work, recent evidence has emerged on the role of TRPA1 in mediating noxious cold transduction at a visceral level and in contributing to visceral thermosensation and thermoregulatory responses (95). For example, Fajardo et al. (95) observed that activation of a large population of cultured rat vagal neurons in response to cooling stimuli (temperatures as low as $10^{\circ} \mathrm{C}$ ) could be arrogated when the same cells were treated with specific TRPA1-antagonists. The pharmacological profile of these vagal cold-sensitive neurons revealed that the majority of these neurons expressed both TRPA1 and TRPV1 channels, indicating these neurons (and related ion channels) to be primarily involved in coding nociceptive responses potentially resulting from harmful thermal stimuli applied to the viscera (95). The same authors also observed an impairment in cold-defense responses in TRPA1 knock-out mice in vivo. Altogether, this pharmacological and behavioral evidence indicated that TRPA1 could also represent a major contributor to cold sensing in mammalian vagal afferent fibers (95).

At this point, it is however important to highlight that, while TRPA1 has been put forward as a potential key player in the molecular transduction of noxious cold (at least in mice), this fact does not directly imply that homologues channels in humans and other primates could sub serve temperature transduction within the same noxious cold temperature range (47). Recent investigations on the temperature sensitivity of TRPA1 across different species (46) have indeed shown that the temperature sensitivity of TRPA1 could be species-dependent, as a result of an evolutionary divergence driven by different requirements for survival (189).

In their recent work, Chen et al. (46) have shown that the TRPA1 ion channel can alternatively be a heat sensitive channel in invertebrates and ancestral invertebrates (e.g. fly and snakes), a noxious cold sensitive channel in rat and mouse, and a temperature insensitive channel in human and rhesus monkey. It therefore follows that, as also highlighted by Taylor and Chen (47), observations arising from mice models on ion channels temperature selectivity could be limited in their translation potential to humans. Hence, until such findings are replicated in species (e.g. monkeys) whose ion channels share very similar pharmacological profiles with humans (18), the translation potential to humans, of findings from mice models, should be carefully considered.

The species-dependent variable importance of TRPA1 in noxious cold transduction (47) has also opened to the question of whether other molecular channels could be involved in gating noxious cold temperatures. In this respect, as a potential complementary channel involved in transducing noxious cold temperature, the identification of the tetrodotoxin-resistant voltage-gated sodium channel Nav1.8 and of its responsiveness to noxious cold in a rat skin-nerve preparation, has provided evidence for the potential contribution of this channel to cold pain detection (312).

Nav1.8 appears to be exclusively expressed in mammalian nociceptive sensory neurons, and has been recently shown to sustain activation at low temperatures (contrary to many other voltage gated sodium channels which show slow voltage dependent inactivation at low temperatures) (312). Loss of Nav1.8 in rat dorsal root ganglion neurons has been shown to impair responsiveness to noxious cold $\left(10^{\circ} \mathrm{C}\right)$, indicating that action potentials at very low temperatures in dorsal root ganglion neurons could depend entirely on Nav1.8 gating. From a behavioral point of view, mutant mice lacking Nav1.8 show impaired cold nociception behavior during cold-plate 
tests (312). Interestingly, Nav1.8 seems to be expressed on TRPM8-expressing neurons, a fact which could explain why ablation of TRPM8 neurons can lead to loss of innocuous as well as noxious cold sensitivity (183). The relevance of these findings is in showing the importance for thermosensation of other more traditional channels than TRPs, such as potassium and sodium channels, which are well known to contribute to the regulation of neurons' membrane resting potential and to the threshold voltage for the generation of the action potential. Changes in the activity of such potassium and sodium channels can influence the effect of a depolarizing current (regulated by TRP channels activity), hence the thermal sensitivity of a neuron (297).

In summary, along with the potential role of TRPM8 (183), the above reviewed evidence provides insights on the potential physiological and behavioral role of the TRPA1 and Nav1.8 channels in contributing to mammalian (mice) noxious cold transduction (temperatures $<15^{\circ} \mathrm{C}$ ). However, it has to be stressed that, regardless of whether or not alternative channels (e.g. TRPA1) to TRPM8 play a key role in noxious cold transduction, the species-specific evolutionary diversification of some thermo-sensitive TRP ion channel should be taken into consideration when translating findings from animal models to human temperature transduction. In this respect, endorsing animal models (e.g. primates) with pharmacological profiles analogous to those of humans would be therefore more appropriate to clarify the molecular mechanisms underlying noxious (as well as non-noxious) cold transduction in humans.

\section{Summary of the properties and function of thermo-sensitive TRP ion channels}

Great advances have been made in the past 20 years with regards to the discovery and characterization of the properties of thermo-sensitive TRP ion channels, i.e. the putative thermometers in our body (299). While the structural and functional properties of these channels are still partly obscure, a number of channels have been identified to gate temperaturetransduction within specific temperature ranges.

Remarkably, a feature of mammalian thermo-sensitive TRP ion channels is that these channels could play a role in both thermal detection as well as in the modulation of thermoregulatory responses. As that, highlighting the intertwined nature of behavioral and autonomic temperature regulation, both from an anatomical and physiological point of view. Nevertheless, while evidence has been provided on the genetic, physiological and behavioral impact that thermo-sensory TRP ion channels exert on mammalian autonomic and behavioral function, the picture that emerges from the analysis of the molecular mechanisms underpinning temperature transduction and skin thermal sensations is complex and still partially blurry.

Numerous outstanding questions still await experimental answers, and it is hoped that the wait will not be long until the molecular mechanisms of temperature transduction will be fully clarified. The need for such evidence is driven by the potential clinical implications of such knowledge in the context of human pathophysiology. Pharmacological manipulations of TRP ion channels involved in thermoreception and temperature regulation is indeed a promising avenue for the development of more effective strategies in the induction and management of therapeutic hypothermia, a remarkably effective, although challenging, method of neuroprotection from ischemia (97). Furthermore, the emerging evidence on the involvement of thermo-sensory TRP channels in airway diseases (e.g. asthma) (214) as well as in dry eye disease $(152,240)$ highlights the need of deepening the knowledge on these molecular transducers of environmental stimuli, in order to improve therapeutic approaches to human diseases. 


\section{Summary of the neurophysiological bases of temperature sensation}

In summary, the above presented evidence indicates that, from a neuroanatomical point of view, the specific thermosensory pathway which allows humans to peripherally encode and centrally process skin thermal sensations comprises of: first order thermo-sensitive afferent fibers (i.e. skin thermoreceptors); second order lamina I spino-thalamic neurons projecting to the posterior part of the ventral medial nucleus of the thalamus (VMpo); third order neurons located in posterior part of the ventral medial nucleus of the thalamus (VMpo) projecting to the posterior insular cortex. Functionally, this neurophysiological pathway sub serves peripheral and central mechanisms underpinning our ability to characterize both the discriminative and affective components of skin thermal sensations in the context of thermal behavior. Interactions with other cortical (e.g. somatosensory and cingulate cortices) and sub cortical regions (e.g. nucleus of the tractus solitarious, parabrachial nucleus and the hypothalamus) likely contribute to enrich the polymodal nature (e.g. thermal-tactile interactions) underpinning human thermo-sensory experiences.

From a molecular point of view, one of the most recent and important advances in our understanding of skin thermal sensations has to do with the molecular characterization of thermoreception. The temperature-dependent generation of action potentials in thermo-sensory neurons has been shown to rely on the presence of specific transmembrane proteins which, by gating changes in ion fluxes across the plasma membrane in a temperature-dependent manner, contribute to generating action potentials at the nerve fiber level in response to temperature stimuli (297). Such protein receptors have been recently identified as to belong to the Transient Receptor Potential (TRP) ion channels family. Several channels have been identified to contribute to the highly sensitive mammalian thermal spectrum.

Figure 19 presents a visual summary of the molecular, neuroanatomical, and functional structures and properties underpinning human skin thermal sensations.

At this point, having outlined the current knowledge on the neurophysiological mechanisms underpinning our remarkable ability to finely perceive thermal changes in our body and surrounding environment (272), it appears important to highlight that sensing temperature is not the only somatosensory mechanism to contribute to autonomic and behavioral thermoregulatory responses in humans. The ability to sense skin wetness and humidity (i.e. hygroreception) plays indeed an important role in modulating behavioral and autonomic thermal adaptations.

From a behavioral standpoint, it has been repeatedly shown that increases in ambient humidity and skin wetness have a detrimental effect on thermal comfort (109) and related thermal behavior (262). The extent of this effect seems to be dependent on factors amongst which the presence of specific health conditions (e.g. changes in ambient humidity appear to influence thermal comfort to a greater extent in individuals suffering from rheumatic pain than in the healthy population) (280). From an autonomic standpoint, the degree of sweat-induced physical skin wetness (i.e. the proportion of the body covered by moisture at skin temperature) (112) has been shown to modulate sweat gland activity via a progressive suppression of sweat output (i.e. hidromeiosis) with increasing levels of wet skin (223). This process has been suggested to potentially contribute to maintaining sweating evaporative efficiency (41). As well as for thermoregulatory adaptations, sensing wetness has been also shown to be essential for other regulatory and behavioral mechanisms. Being able to sense variations in ocular wetness is indeed critical to control the lacrimation reflex and protect the ocular surface (153). Furthermore, sensing and discriminating tactile roughness and wetness is key to control precision grip (7) and manipulate objects (4).

Despite its importance in the context of behavioral and physiological function (see Thermoreception and Hygrosensation paragraph), the neurophysiological bases of human skin wetness perception have only recently started to be unveiled.

The following final sections of this review will present the current state of the knowledge on the peripheral and central neurophysiological mechanisms underlying human hygrosensation. As the 
psychophysics of skin wetness perception have been recently reviewed in details elsewhere (see Filingeri \& Havenith, 2015) (101), these will only briefly be outlined below.

\section{Psychophysics of skin wetness perception}

Being provided with humidity receptors (i.e. hygroreceptors) is an anatomical and functional feature that numerous animal species can claim to possess. Amongst these, insects are a prominent example $(10,105,199,288)$. However, while feelings of wetness and humidity induced by contact with fluids and exposures to different environments are common experiences in human life, it is surprising that as human beings we seem not to have developed specific skin receptors for the sensation of humidity and skin wetness (53).

This apparently paradoxical condition, which see the occurrence of a perception without the presence of a specifically evolved sensory receptor, has resulted in numerous scientists being attracted by the challenge of investigating how humans could sense skin wetness. As early as 1900 , scientists attempted to explore what psychophysical mechanisms could underpin this perception (13). Amongst these early studies, with his famous "synthetic experiment", Bentley (13) was the first one to rigorously test the perception evoked by dipping a sheath-covered finger into warm, lukewarm and cold water in blindfolded participants. Bentley observed that, when in contact with water, participants could experience a clear perception of wetness a through their sheath-covered finger, despite no direct contact between the skin and water was present. Interestingly, this synthetic perception was more pronounced when the water was cold than when it was warm. Surprisingly, when these blindfolded participants were informed that during the experiment, no direct skin contact with water occurred, they initially refused to believe that their finger had not been wet at any point in time (13). These pioneering observations led Bentley (13) to conclude that the perception of skin wetness could therefore result from the blend of light pressure/touch and cold sensations, as likely experienced by his participants during the synthetic contact with water. Both the touch and temperature sense were therefore for the time suggested as to be determinant in characterizing this particular somatosensory experience.

Bentley's early work (13) had set the starting point for those future studies which, in years to come, will be investigating the psychophysical bases of skin wetness perception $(1,15,16,71$, 98-104, 109, 120, 191, 196, 227, 284, 285). Alternatively exploring the two main sides of the perception of skin wetness [1) perception resulting from skin's contact with wet stimuli) $(1,15,16$, $71,98-104,109,120,191,196,227,284,285)$ 2) perception resulting from the active production of sweat $(100,109,120,191)]$, these studies have contributed to increase our understanding of the psychophysical bases of human skin wetness perception.

It appears to be widely accepted that perceiving the wetness experienced when the skin is in contact with a wet surface or when sweat is produced (16) is a learnt ability. In the presence of physical wetness, thermal (i.e. heat transfer) and tactile (i.e. mechanical pressure and skin friction) inputs, as generated the interaction between skin, moisture and (if donned) clothing (109), could be used to determine the presence and degree of skin wetness, through a complex multisensory integration (89), which is shaped and refined by repeated exposures to the same wet stimuli. In this context, skin thermal and tactile sensitivities seem therefore to play an important role in the ability to sense skin wetness (101).

For example, humans have been shown to readily and accurately discriminate between higher and lower levels of skin wetness with remarkable sensitivity (i.e. levels of wetness which differ as little as of $0.04 \mathrm{ml})(16,17,285)$. Thermal (cold) sensory inputs have been proposed to play a primary role in driving this finely tuned ability to perceive skin wetness. In support of this, and in line with the hypothesis of sensory acquisition via learning mechanisms, it has been suggested that detecting the presence of moisture, and thus wetness, on the skin surface could result from associating the cold sensations evoked by the drop in skin temperature occurring during the 
evaporation of moisture from the skin, to the perception of skin wetness $(1,15,71)$. The possibility to sense skin wetness via associative processes, has been recently confirmed when cold-dry stimuli able to reproduce skin cooling rates similar to the ones occurring when the skin is wet, evoked wetness perceptions in blindfolded participants (Fig. 20) (102, 103). Further evidence in support of the above has been provided when in another recent study, when warm-wet stimuli statically applied to the skin were not perceived as wet by blindfolded participants (104). Altogether, while supporting the hypothesis for which as humans we associate certain levels of skin cooling and cold sensation to the perception of wetness, these results have also highlighted the key role played by cold sensitivity per se in determining the perception of skin wetness.

That temperature cues in the form of coldness could be primarily used within the sensory processing for skin wetness, does not cancel out the fact it is common experience that wetness can also be sensed in the absence of coldness and temperature stimuli (i.e. during contact with lukewarm water; while sweating in hot humid environments). This simple observation has led to a number of investigations exploring how other somatosensory stimuli (e.g. mechanical and tactile) could therefore contribute to the perception of skin wetness in the absence of cold sensations $(16,98,103)$. As a result of such studies, evidence has emerged in support of the fact that when thermal cues are insufficient, individuals seems to rely on mechanical and tactile inputs (e.g. stickiness) to sense skin wetness. This is particularly true under conditions of sweat-induced skin wetness $(16,98,103)$. For example, under conditions of sweat-induced skin wetness, and especially when wearing clothing, skin wetness perception has been shown to be driven primarily by tactile stimuli in the form of intermittent mechanical interaction between skin, sweat and clothing $(100,109,120,191)$. Interestingly, these findings are in line with what observed with for the mechanisms involved in the ability to sense skin wetness when in contact with external stimuli, and have therefore led to the current view for which the integration of specific thermal and tactile sensory cues is considered critical in order to experience the perception of skin wetness, irrespectively of the modality by which wetness is experienced (i.e. passive contact with wet stimuli or active sweat production) (87).

Increasing the understanding on how specific somatosensory cues (i.e. thermal and tactile) underlie the ability to perceive skin wetness, has also to led to the investigation of the potential regional variability in skin wetness sensitivity across the body, and of its relationship with what known with regards to the regional differences in sensitivity observed in other somatosensory modalities (e.g. thermal sensitivity) $(1,99,100,109,120,191)$. As a result, we now know that, during the contact with external stimuli, as well as a result of sweating, skin wetness can be perceived to a larger extent on certain regions of hairy skin (e.g. the torso) than on other body parts (e.g. limbs). Specifically, mapping the regional variations in cold-induced skin wetness sensitivity across the human torso (Fig. 3C) has revealed not only that areas of significantly higher sensitivity are present (e.g. lower back), but that also, these correspond to regions presenting greater cold sensitivity (85). Finally, within the context of regional variability on wetness perception, it is worth noting that, while limited psychophysical evidence is available on whether skin wetness is perceived differently between hairy and glabrous skin, a recent study from this author has shown that, owing to its potentially higher thermal sensitivity, hairy skin presents higher wetness sensitivity than the glabrous skin of the finger pad (98).

In summary, this brief review of the psychophysical evidence available to date, highlights the fact that, in the absence of specific skin hygroreceptors, sensory inputs in the form of temperature and tactile cues, are likely to support our remarkable ability to sense skin wetness. At this point, appraising the fundamental relevance of thermal and tactile somatosensory cues has opened to the question of how peripheral (i.e. sensory nerve fibers) and central nervous structures (i.e. cortical and sub-cortical areas) interact in processing the perception of skin wetness in humans. In this respect, as it is only recently that attempts have been made to determine the neural mechanisms underpinning human hygrosensation, the state of the knowledge on the neurophysiology of skin wetness perception is still in its early infancy. The following paragraph will present an overview of the results of these recent attempts. 


\section{Neurophysiology of skin wetness perception}

To date, only two human studies have tackled the problem of what peripheral and central neural mechanisms (in the form of activity in first order thermo- and mechano-sensitive neurons and of central perceptual integration) could be involved in sensing wetness and humidity on our skin (98, 205). These studies have shown that it reasonable to hypothesize that the peripheral mechanisms of thermo- and mechano-sensory integration could provide the neurophysiological bases for the first level of integration required for being able to sense skin wetness. However, these studies have also shown that what is still far from being fully understood is how such specific sensory inputs are coded and processed centrally, in order to form a coherent sensory percept such as the perception of skin wetness. While providing initial insights on the neurophysiology of skin wetness perception in humans, these recent studies have indeed highlighted the lack of mechanistic investigations specifically targeting the neural mechanisms underlying this complex perception. Nevertheless, comparative results from recent animal studies investigating the molecular bases of humidity sensation in hygroreceptor-lacking organisms, have contributed to expand our understanding of the mechanisms involved in the central integration of skin wetness perception. In light of the similarities between human and animal hygrosensation, these recent findings have open to the hypothesis that potentially universal hygrosensation strategies could exist and be shared by a wide range of species (including humans).

The significance of unveiling the neurophysiological mechanisms of human hygrosensation is twofold. From a fundamental point of view, determining the exact sensory mechanisms involved in skin wetness perception represents a way to gain conceptual and functional understanding of somatosensory integration under normal brain function. Clinically, as numerous neurological disorders, amongst which multiple sclerosis, are characterized by the development of sensory abnormalities in the form of burning sensations across the body, paradoxical wetness sensations, and decreased detection thresholds for cold and warm temperatures $(132,136,236,283)$, it is clear how increasing the fundamental knowledge on human somatosensory integration could improve our understanding of the link between neurological disease and altered somatosensory function.

The central and peripheral neurophysiological mechanisms of skin wetness perception in humans, along with comparative evidence on animal hygrosensation, will be presented below.

\section{Peripheral and Central mechanisms}

It is not surprising that as humans, we tend to associate coldness and light touch to the idea of a typical wet stimulus. The biophysical mechanisms involved in the physical experience of wetness always involve heat transfer (in the form of evaporative cooling) (1), and mechanical pressure (in the form of friction and stickiness) (2). As a result, the peripheral and central neurophysiological events involved in encoding thermal and tactile sensations consistently accompany our experience of skin wetness.

As presented earlier in this review, skin thermal sensations in humans are primarily encoded by first order cold-sensitive myelinated Aঠ sensory neurons (see Peripheral thermo-receptive nerve fibers paragraph). On the other side, tactile sensations resulting from mechanical stimulation of the skin are primarily encoded by first order mechanosensitive $A ß$ sensory neurons. Before further discussing the neurophysiological mechanism of skin wetness perception, it appears necessary at this point to briefly outline the characteristics of first order sensory neurons with mechanoreceptive properties innervating the human skin, in order to provide the non-specialized reader with an overview of the mechanoreceptive units which could transduce those tactile sensory inputs involved in the perception of skin wetness. For a more detailed account of human 
mechanotransduction the reader is referred to the work of McGlone et al. (207) and Saal and Bensmania (258).

Mechanical stimulation of human hairy skin result in tactile stimuli which are transduced and encoded by different mechanoreceptors: 1) slowly adapting myelinated type I Merkel cells (Aßsensory neurons responding to static indentation of the skin; conduction velocity: $16-100 \mathrm{~m}^{-1}$ ) and type II Ruffini endings ( $A ß$ sensory neurons primarily responding to skin stretch; 2 ) rapidly adapting myelinated Pacinian-type units ( $A ß$ sensory neurons primarily responding to the initial and final contact of a mechanical stimulus with the skin), hair units ( $A ß$ sensory neurons primarily responding to hair deflection) and field units (Aß-nerve fibers primarily responding to skin indentation); 3) intermediate adapting unmyelinated $\mathrm{C}$-tactile units (C-nerve fibers primarily responding to slow, gentle touch) $(207,232,294)$. First order $A ß$ sensory neurons with mechanosensitive properties present conduction velocities in the range of $16-100 \mathrm{~ms}^{-1}$; while C-type sensory neurons present conduction velocities of $<2 \mathrm{~ms}^{-1}$. First order mechanosensitive neurons lie in the dorsal root and trigeminal ganglia of the spinal cord from which they extend sensory afferents to the skin, where they end either in the form of free nerve endings or with specific corpuscles (i.e. specialized cells).

In light of both the psychophysical results on the perception of skin wetness and of the above referenced characteristics of thermosensory and mechanosensory first order sensory neurons, it could be proposed that the central integration of thermo- and mechano-sensory afferents, as encoded by first order cold- and mechano- sensitive myelinated A-type sensory neurons, could provide the neural foundations for humans' ability to sense wetness in the absence of skin hygroreceptors. Evidence for the fact that the central integration of inputs from peripheral thermal and tactile A-type nerve afferents could represent a specific sensory strategy developed by humans to sense wetness, has been recently provided in one of this author's studies (98).

In this study (98), individuals' ability to sense skin wetness was first characterized with psychophysical methods. While undergoing a quantitative sensory test during which subjective perceptions of wetness were recorded, thirteen blindfolded male participants exposed the hairy skin of their forearm and the glabrous skin of their left index finger pad to a static and dynamic contact with cold-wet (i.e. $25^{\circ} \mathrm{C}$ ), neutral-wet (i.e. $30^{\circ} \mathrm{C}$ ) and warm-wet (i.e. $35^{\circ} \mathrm{C}$ ) stimuli characterized by the same moisture content (i.e. $80 \mu \mathrm{lcm}^{-2}$ ). In line with previous psychophysical evidence, individuals experienced the cold-wet stimuli as to be significantly wetter than neutral and warm ones. Such effect was particularly pronounced when participants where in static contact with the stimuli (note: under this condition it would be expected that only low-threshold mechanoreceptors responding to the static pressure of the stimulus against the skin would be activated). During the following dynamic interactions with the wet stimuli, a significant increase in the extent of wetness experienced by the participants was recorded for all stimuli and irrespectively of their temperature (note: under this condition it would be expected that additional low-threshold mechanoreceptors responding to skin friction, vibration and lateral stretching would be also activated).

These initial results confirmed the role that skin thermal (i.e. cold sensitive) and mechanosensory (i.e. responding to skin friction, vibration and lateral stretching) afferents played in driving individuals' ability to sense wetness. From a neurophysiological standpoint, these results therefore indicated that A-type sensory neurons (i.e. cold thermoreceptors and mechanoreceptors) would likely encode those afferent inputs required to sense wetness. At this point, to confirm that such peripheral integration would be essential to determine human ability to perceive skin wetness, we went further investigating whether artificially dampening afferent activity in A-type sensory neurons (98), and therefore reducing cold and mechano sensitivity of the skin, would in turn significantly impair the ability to perceive skin wetness in our otherwise healthy thirteen individuals. To reach this aim, the same participants repeated the quantitative sensory test described earlier, however only after a selective reduction in the afferent activity of their cutaneous cold- and mechanosensitive A-type sensory neurons was determined. A modified local compression-ischemia protocol was used to induce a dissociated reduction in myelinated A- 
fibers afferent activity $(81,310)$, with no concurrent effects on C-fibers function (i.e. primarily subserving conscious warmth and pain sensitivity) (292). The results of this quantitative sensory test indicated that, while participants interacted with the same cold-wet, neutral-wet and warm-wet stimuli used during the test performed without dampening A-fibers function, the artificially induced reduction in skin cold and mechano-sensitivity resulted in a significant impairment in the ability to sense wetness in all participants, both on hairy and glabrous skin sites (98).

Altogether, these psychophysical and neurophysiological findings provided robust evidence for the fact integrating afferent inputs from peripheral myelinated A-nerve fibers sensitive to cold and mechanical stimulation of the skin, is an essential mechanisms to sub serve the central neural processing underpinning humans' ability to sense wetness (98). This has allowed the development of a first conceptual model which describes the neurophysiological pathways which could allow humans to specifically sense wetness on their skin (Fig. 21) (98).

The main working assumption behind this model is that the human brain could infer about the perception of wetness in rationale fashion (118), by integrating sensory inputs conveyed within the thermosensory (cold) and mechano-sensory afferent pathways, and then assessing their reliability in relation to the nature of stimulation (i.e. dry or wet; cold, warm or neutral; static or dynamic) and to prior experiences (with wet stimuli), in order to determine whether skin wetness is to be perceived.

Let's take the example of experiencing warm-wetness (Fig. 21C \& D). According to the proposed model, in the presence of warm-wetness, tactile and mechanical inputs (e.g. skin friction and stickiness resulting from dynamic interactions with moisture) will have a greater role than thermal stimuli in determining whether warm-wetness will be sensed, primarily as a result of the absence of the specific thermal component (i.e. coldness) which has been shown to be the main contributor to the sensory pathway for wetness. In line with this example, the likelihood of experiencing cold-wetness is therefore higher than that of warm and neutral wetness (Fig. 21C, $D, E \& F)$, both when dynamic mechanical interactions between moisture and skin are absent, as well during dynamic contacts. In either case, the prevailing thermal (cold) inputs alone (Fig. 21G), or the combination of this with additional mechanical inputs (Fig. $21 \mathrm{H})$, will be indeed always present.

While this conceptual neural model could sound rather theoretical at first, it is important to highlight that psychophysical evidence (see Psychophysics of skin wetness perception paragraph) provides numerous examples in support of this proposed perceptual framework.

For example, it has been reported earlier in this review that when asked to discriminate about the wetness properties of a wet material during haptic exploration (16), individuals have been shown to rely more on mechanical cues (e.g. stickiness resulting from the adhesion of a wet material to the skin) to aid them in the perception of wetness, if thermal cues (e.g. thermal conductance of a wet material) are limited or absent. Similarly, tactile cues experienced when actively sweating under conditions of limited evaporative cooling, could contribute to individuals' ability to sense wetness when sweating $(109,120,191)$. According to the neural framework for the perception of wetness, under those conditions, mechanical cues could be indeed more salient than their thermal counterparts (i.e. warm sensations). This has been recently demonstrated in one of this author' study (100), in which the alteration of the interactions skin-sweat-clothing under conditions of sweat-induced skin wetness, significantly modulated the perception of skin wetness, independently of the level of physical skin wetness. Finally, in light of this neural model, perceptual illusions such as the one where skin wetness is experienced as a result of cold-dry stimulation $(102,103)$, could be explained and interpreted as the result of a sensory system which relies on rational integration mechanisms which are based on specific sensory inputs (e.g. coldness), and which are selectively used to characterize a specific sensory experience. The synthetic nature of wetness is highlighted by the simple, but powerful observation that in the presence of specific thermal and tactile sensory inputs, wetness can be experienced, regardless of whether the inputs are produced by actual contact with moisture stimuli (e.g. see contact with 
col-dry stimuli) $(102,103)$. Similarly, in the absence of these specific sensory inputs (e.g. see during static contact with a warm wet stimulus or during limited tactile interaction with clothing during sweat-induced skin wetness) $(100,104)$ the perception of wetness can be missed, even if actual contact with moisture occurs.

At this point, an obvious question will arise: why would the brain infer about the perception of wetness in such fashion? Perceptual learning and somatosensory decision making could be candidate answers to this question (246).

As the skin seems not to be provided with hygroreceptors (53), it is reasonable to hypothesize that the perception of skin wetness is shaped by sensory experience (i.e. we learn to associate the sensations experienced when in contact with moisture to the actual perception of skin wetness). It could be argued that in the absence of a specific hygroreceptors, humans have learnt to associate the experience of wetness to secondary sensory inputs (thermal and tactile), by retaining a neural representation of a "typical wet stimulus" $(98,101)$ which is generated and shaped by repeated sensory experiences (i.e. exposures to wet stimuli). When the skin is physically wet, inputs from sensory sub-modality such as thermal and touch, could be transformed into a sensory reference frame (133) which could ultimately determine the neural representation of skin wetness perception.

Increasing evidence for the fact that our sensory systems could operate according to rational associative and probabilistic assumptions has recently arisen and has opened to the endorsement of Bayesian modelling within the study of the neuroscience of human perception (181).

Bayesian perceptual frameworks are based on the assumption that sensory systems acquire knowledge on the properties of the surrounding environment and use this information to generate sensory priors which are then used to infer about the properties of specific stimuli (118).

Priors allow sensory stimuli which are generally multimodal (i.e. involving different sensory cues), noisy and ambiguous, to be efficiently "filtered" and used appropriately to the context. As a result, it has been repeatedly shown that sensory systems perform on-line tasks which, as supported by the use of implicit sensory knowledge, help predicting the underlying causes for a sensory observation in a near-optimal fashion (200). Human sensory systems (e.g. visual system) appear to integrate the different sensory cues associated with an external stimulus in this fashion (94, 303). By assessing the reliability and variability of each sensory cue involved in a sensory experience in a computational fashion, sensory systems have been shown to contribute to our ability to efficiently infer about current multimodal perceptions $(94,303)$.

In light of its multimodal nature (i.e. blend of thermal and tactile stimuli), the perception of skin wetness is therefore currently proposed as to result from such inference processes as operated by the human brain in accordance to the specific neural representation of a typical wet stimulus (98). In order to determine whether physical wetness is present/absent and whether the perception of skin wetness has to be experienced, the human brain could therefore take into account the reliability of the sensory information provided by each sensory modality (i.e. thermal and tactile), and infer the most likely percept (98).

While the conceptual model presented above could provide working hypotheses on the computational properties of our somatosensory system, the same provides limited evidence on both the potential levels of the neuroaxis where such sensory integration process could occur, as well on how neuronal populations could code and process such information.

As to date, the only direct evidence on potential neuronal populations involved in detecting skinwetness related inputs in humans, has arisen from the only study in which activity in first-order mechano-sensitive neurons innervating the skin was recorded via microneurography in awake humans, during the application of droplets of water $(0.05 \mathrm{ml})$ on the neurons' receptive fields on the skin (205). Mechano-sensitive Aß-nerve fibers, and specifically rapidly adapting Pacinian and 
hair mechanoreceptive units, were indeed observed to primarily encode the tactile components of water droplets when these were applied on the unit's receptive field (205), a fact, which further support the potential key role that peripheral myelinated nerve fibers could play in sub-serving skin wetness perception. Indirect evidence on the role of myelinated first-order thermoreceptive neurons could be also derived by those animal studies in which water droplets at different temperatures were shown to trigger activation of first order thermoreceptors (e.g. in the cat's tongue) $(313,314)$.

While the lack of direct neural recording in humans highlights the need for future studies specifically targeting this topic, our current knowledge on the cortical and sub-cortical regions involved in sensory integration and processing of temperature and tactile stimuli could help identifying the potential neuronal populations involved in the perceptual inference underpinning the perception of skin wetness.

We have long known that the primary and secondary somatosensory cortices are cortical regions involved in integration of somatosensory and tactile afferents. We have recently learnt about the involvement of the posterior insular cortex in cold and warm temperatures integration (62). Finally, we are also aware that the posterior parietal lobe is a cortical region concerned with integrating the different somatic sensory modalities necessary for perception (206). In light of this cortical organization, it could be therefore speculated that the neural processes involved in the perceptual inference underpinning the conscious perception of skin wetness could be ultimately coded by neuronal populations within these cortical regions (98). The primary and secondary sensory cortices as well as the insular cortex could integrate initial tactile and thermo-sensory information arising from the contact with physical wetness. These information could be then transferred to nearby unimodal association areas and elaborated by multimodal association areas such as prefrontal, parietotemporal and limbic cortices (where neural patterns of activity for typical wetness stimuli could be retained) (169), where behavior-oriented perceptual decision making is computed $(62,246,290)$.

It deserves mention that studies investigating the interplay of sub-modalities in cutaneous tactile sensibility to object manipulations (e.g. populations of different mechanosensory afferents coding shape, texture, motion, stretch and vibration perceptions), have recently indicated that tactile cues from different classes of first order mechano-sensitive neurons could already be combined in different sub-cortical rely nuclei (e.g. cuneate nucleus and ventro posterior lateral nucleus of the thalamus) before reaching the somatosensory cortex (258). While this evidence for subcortical sensory integration in touch processing is not in contrast with the idea that sub-modalities integration in wetness perception could be primarily processed at a cortical level, these recent results open to the question of whether similar sub-cortical convergence of skin wetness-induced thermal and mechanical inputs could occur before higher cortical regions are engaged. It is likely that, in line with other bodily feelings (72), a combination of sub-cortical and cortical convergence in the processing the perception of skin wetness occurs. In this respect, what emerges from recent studies (98) is that convergence of somatosensory inputs is clearly and critically important for the brain to infer about the perception of wetness. In line with Bayesian perceptual inference (94), the combination of two or more sensory afferents during sensory estimation indeed results in more accurate percepts than would be available from either afferent alone (181). With respect to skin wetness perception, this is particularly evident when considering that, although cold and mechanical inputs can individually evoke and modulate the perception of skin wetness $(102,103)$, their dynamic combination results in an even more powerful estimate of the perception of wetness (98).

While numerous outstanding questions still await experimental answers, the development of the conceptual model of human skin wetness perception presented above, has highlighted the possibility to characterize the central mechanisms of human hygrosensation with computational modelling. If used in combination with neural imaging techniques, it is predicted that these experimental and statistical techniques could help in rapidly developing a better understanding of the neural bases of skin wetness perception. In this respect, comparative results from animal 
studies exploring the molecular mechanisms of humidity sensation in hygroreceptor-lacking organisms (e.g. the roundworm Caenorhabditis elegans) (257), have shown some remarkable similarities in the hygrosensation strategies developed by humans and other animal species. These observations should be taken into account when considering the possibility to use animal models in combination with experiments in humans, as a way to further explore this area.

As to date, the only indirect evidence on the potential molecular mechanisms underpinning human hygrosensation arises from studies investigating the neural mechanism controlling ocular wetness and the pathophysiology of dry eye syndrome $(153,240)$. Interestingly, the autonomic mechanisms controlling basal tearing and ocular wetness have been shown to be dependent upon the activity of cold-sensitive sensory neurons responding to corneal-cooling, which results from the evaporation of tears from the ocular surface. Remarkably, the expression of cold sensitive TRPM8 ion channels on corneal sensory neurons, has been shown to be essential for regulating basal tear flow, and therefore ocular wetness (240). For example, figure 22 shows responses of mice corneal neurons to cooling ramps and menthol, as well as dependence of tear secretion rate on corneal temperature, as recorded in wild type mice expressing TRPM8 ion channels, in mutant mice presenting a reduced expression of TRPM8 ion channels and in TRPM8-deficient mice.

By providing evidence for the role of cold sensitive TRPM8 ion channels in controlling ocular wetness, these results have highlighted the possibility that the same processes involved in the behavioral ability to consciously sense skin wetness (i.e. integration of cold afferent inputs during the contact with moisture) could be similarly used by the human body to autonomically regulate tear flow via sensing changes in ocular wetness resulting from evaporation-induced corneal cooling. This intriguing possibility further strengthens the evidence in support of the potential neurophysiological and molecular mechanisms involved in sensing skin wetness in humans.

It should be however noted that, as the analysis of the molecular mechanism of temperature sensation in humans (see Thermo-sensitive Transient Receptor Potential (TRP) ion channels paragraph) has shown that our understanding of temperature (as well as mechano-) transduction in humans is still very limited, whether temperature gated TRP channels (along with mechanically activated Degenerin/Epithelial sodium channels) $(202,293)$ could be involved in human hygrosensation, has still to be clarified. If it was shown that pharmacological manipulation of temperature and mechanically-activated ion channels could disrupt/rescue human ability to sense humidity and wetness, the implications of this findings could be clinically relevant. Along with the possibility to improve the treatment of diseases such as dry eye syndrome (240), these investigations could provide 1) insights on the potential pathological mechanisms involved in the altered somatosensory function observed in patients suffering from neurological disorders such as multiple sclerosis or polyneuropathies; 2) working hypotheses to be used in the development of new treatments to improve/restore normal sensory function in pathological conditions (299).

In summary, the experimental evidence currently available shows how little we know about the neural bases of skin wetness perception and of its somatosensory integration. Nevertheless, working hypotheses and conceptual models have started to be produced. These efforts have shown that humans could have developed a specific hygrosensation strategy which allow the transduction of biophysical processes (i.e. thermal and mechanical changes induced by the presence of moisture on the skin) into neurophysiological signals (i.e. afferents inputs from thermo- and mechano-sensitive ion channels and nerve fibers) which are then psychophysiologically integrated (i.e. perceptual inference operated by cortical and sub-cortical somatosensory and association areas) in the context of our previous sensory experience (Fig. 23). This hygrosensation strategy could underpin our remarkable ability to sense skin wetness and humidity in the absence of specific skin hygroreceptors. The fact that this hygrosensation strategy seems to be shared by other hygroreceptor-lacking animal species, could indicate that wetness and humidity detection could be based on universal mechanisms across the animal kingdom which could carry evolutionary significance in the context of maintaining homeostasis and ensuring survival. 


\section{Conclusion}

Without doubt, the ability to adjust our thermoregulatory behavior represents the most effective mechanism to maintain thermal homeostasis and ensure survival in the diverse and potentially hostile thermal environments that we face on this planet. Remarkably, our thermal behavior is based entirely on our ability to detect temperature and humidity variations in our internal (i.e. body) and external environment, via sensing changes in skin temperature and wetness.

In the last 30 years, we have seen a significant expansion of our understanding of the molecular, neuroanatomical and neurophysiological mechanisms that allow humans to sense temperature and humidity. The discovery of temperature-activated trans-membrane ion channels which gate the generation of action potentials in thermo-sensory neurons in response to temperature stimuli; along with the neuroanatomical and neurophysiological characterization of the spino-thalamocortical tract which sub serves temperature integration and processing; and the development of neural models for the perception of skin wetness and humidity, are only some of the recent advances which have provided incredible insights on how biophysical changes in skin temperature and wetness are transduced into those neural afferent signals which constitute the physiological substrate of skin thermal and wetness sensations. Nevertheless, while emerging evidence is now available on the neurophysiological and behavioral impact that afferent activity in the ascending thermo-sensory pathway exerts on mammalian autonomic and behavioral thermosensory function, the picture that emerges from the analysis of the neurobiological mechanisms underpinning skin thermal and wetness sensations is complex and still far from being conclusive, particularly in humans.

While genetic and behavioral assays with animal models have greatly increased our understanding of the neurophysiology of mammalian temperature transduction in the context of behavioral thermoregulation, extrapolating human thermal behavior based on such findings is a risky intellectual exercise, which requires further human-based experimental evidence. As to date, we still face a lack of mechanistic evidence in humans on how temperature integration is influenced by internal (e.g. body core temperature), external (e.g. mean skin and ambient temperature) and emotional states (e.g. thermal pleasantness and the valence assigned to thermal stimuli), and on how these interactions determine the magnitude of a thermal sensation, influence individual thermal preference and ultimately shape human thermal behavior across the life span.

The possibility to functionally map the connections and circuitry of afferent thermo-sensory neurons in humans while monitoring their activity and their impact on behavioral and autonomic function in vivo will be therefore critical in order to explore how temperature sensations drive human behavioral and autonomic thermoregulation. The implications of these findings will be of fundamental, applied and clinical significance.

Increasing the understanding of how afferent signals ascending the thermosensory pathway can modulate both thermal behavior and efferent thermoregulatory responses under normal brain function, is critical to accurately determine how such integration is disrupted in those neurological conditions, amongst which multiple sclerosis, which see the concurrent presence of afferent thermo-sensory deficits (e.g. sensory abnormalities) and efferent thermoregulatory and autonomic dysfunctions (i.e. impaired control of blood pressure, skin blood flow and sudomotor activity).

As well for its role in driving autonomic and behavioral adaptations in the context of maintaining thermal homeostasis, thermo-sensory integration appears to be intrinsically intertwined with central processing of pain, both neurophysiologically and hedonically. Appraising the role that temperature integration plays on the modulation of pain will therefore open to the intriguing opportunity to explore the fundamental interactions between these two important sensory 
percepts in the behavioral regulation of body homeostasis. Ultimately, this could be beneficial in the development of new avenues in the treatment and management of chronic pain.

It is hoped that the many outstanding questions and challenges in the field of thermal neurophysiology will inspire new investigators to further advance our knowledge on human thermoreception and hyrgosensation.

\section{Acknowledgements}

The author would like to acknowledge Prof George Havenith for insightful discussions and mentoring and Dr Victoria Kendrick for invaluable help in proof-reading early versions of this manuscript. The author gratefully acknowledge support for research from the Environmental Ergonomics Research Center at Loughborough University (UK), from Oxylane Research (France), from the Australian Government, Department of Education (Australia) and from the University of California at Berkeley (USA).

\section{References}

1. Ackerley R, Olausson H, Wessberg J, McGlone F. Wetness perception across body sites. Neurosci Lett 522: 73-77, 2012.

2. Adams MJ, Johnson SA, Lefèvre $P$, Lévesque $\mathrm{V}$, Hayward $\mathrm{V}$, André $\mathrm{T}$, Thonnard JL. Finger pad friction and its role in grip and touch [Online]. J R Soc Interface 19;10(80): 20120467, 2013.

3. Almeida M, Hew-Butler T. Pharmacological blockade of the cold receptor TRPM8 attenuates autonomic and behavioral cold defenses and decreases deep body temperature. J Neurosci 32: 2086-2099, 2012.

4. André $T$, Lefevre $P$, Thonnard J. Fingertip moisture is optimally modulated during object manipulation. J Neurophysiol 103: 402-408, 2010.

5. Andrew D, Craig AD. Spinothalamic lamina I neurones selectively responsive to cutaneous warming in cats. J Physiol 537(Pt 2): 489-495, 2001.

6. Attia M, Engel P. Thermal pleasantness sensation: an indicator of thermal stress. Eur J Appl Physiol 50:55-70, 1982.

7. Augurelle AS, Smith AM, Lejeune T, Thonnard JL. Importance of cutaneous feedback in maintaining a secure grip during manipulation of hand-held objects. J Neurophysiol 89: 665-671, 2003.

8. Auliciems A. Thermal sensation and cell adaptability. Int J Biometeorol 58: 325-335, 2014.

9. Bandell M, Story G, Hwang S, Viswanath V. Noxious cold ion channel TRPA1 is activated by pungent compounds and bradykinin. Neuron 41: 849-857, 2004.

10. Bar-Zeev M. Oviposition of Aedes aegypti L. on a dry surface and hygroreceptors. Nature 213: 737-738, 1967.

11. Bautista DM, Siemens J, Glazer JM, Tsuruda PR, Basbaum Al, Stucky CL, Jordt S-E, Julius $D$. The menthol receptor TRPM8 is the principal detector of environmental cold. Nature 448: 204-208, 2007.

12. Becerra L, Breiter H. Human brain activation under controlled thermal stimulation and habituation to noxious heat: an fMRI study. Magn Reson Med 1057: 1044-1057, 1999.

13. Bentley I. The synthetic experiment. Am J Psychol 11: 405-425, 1900.

14. Bergmann Tiest WM, Kappers AML. Tactaile perception of thermal diffusivity. Attention, Perception, Psychophys 71: 481-489, 2009.

15. Bergmann Tiest WM, Kosters ND, Kappers AML, Daanen HAM. Phase change materials and the perception of wetness. Ergonomics 55: 508-512, 2012.

16. Bergmann Tiest WM, Kosters ND, Kappers AML, Daanen HAM. Haptic perception of wetness. Acta Psychol (Amst) 141: 159-163, 2012. 
17. Bergmann Tiest WM. Tactual perception of liquid material properties. Vision Res 109: 178-184, 2015.

18. Bianchi BR, Zhang XF, Reilly RM, Kym PR, Yao BB, Chen J. Species comparison and pharmacological characterization of human, monkey, rat, and mouse TRPA1 channels. $J$ Pharmacol Exp Ther 341: 360-368, 2012.

19. Birklein F, Rolke R, Müller-Forell W. Isolated insular infarction eliminates contralateral cold, cold pain, and pinprick perception. Neurology 65: 1381, 2005.

20. Blix M. Experimentelle Beitrage zur Losung der Frage die specifische Energie des Hautnerven. Z Biol Munich 20: 141-156, 1884.

21. Blomqvist $A$, Zhang E, Craig AD. Cytoarchitectonic and immunohistochemical characterization of a specific pain and temperature relay, the posterior portion of the ventral medial nucleus, in the human. Brain 123: 601-619, 2000.

22. du Bois D, du Bois EF. A formula to estimate the approximate surface area if height and weight be known. Nutrition 5: 303-311, 1916.

23. Bolanowski SJ, Gescheider GA, Fontana AM, Niemec JL, Troblay J. The effects of heatinduced pain on the detectability, discriminability, and sensation magnitude of vibrotactile stimuli. Somatosens Mot Res 18: 5-9, 2001.

24. Boulant JA, Bignall KE. Hypothalamic neuronal responses to peripheral and deep-body temperatures. Am J Physiol 225: 1371-1374, 1973.

25. Boulant JA. Neuronal basis of Hammel's model for set-point thermoregulation. J Appl Physiol (1985) 100: 1347-1354, 2006.

26. Boulant JA. Hypothalamic neurons regulating body temperature. Compr Physiol 2011, Supplement 14: Handbook of Physiology, Environmental Physiology: 105-126. First published in print 1996.

27. Bowsher $\mathrm{D}$. The termination of secondary somatosensory neurons within the thalamus of Macaca mulatta: an experimental degeneration study. J Comp Neurol 117: 213-227, 1961.

28. Brauchi S, Orta G, Salazar M, Rosenmann E, Latorre R. A hot-sensing cold receptor: Cterminal domain determines thermosensation in transient receptor potential channels. $J$ Neurosci 26: 4835-4840, 2006.

29. Brown-Séquard CE. Lectures on the physiology and pathology of the nervous system; and on the treatment of organic nervous affections. Lancet 92: 755-757, 1868.

30. Burke WE, Mekjavic IB. Estimation of regional cutaneous cold sensitivity by analysis of the gasping response. J Appl Physiol (1985) 71: 1933-1940, 1991.

31. Bushnell MC, Duncan GH, Tremblay N. Thalamic VPM nucleus in the behaving monkey. I. Multimodal and discriminative properties of thermosensitive neurons. $J$ Neurophysiol 69: 739-752, 1993.

32. Cabanac M, Massonnet B, Belaiche R. Preferred skin temperature as a function of internal and mean skin temperature. J Appl Physiol 33: 699-703, 1972.

33. Cabanac M. Physiological role of pleasure. Science 173: 1103-1107, 1971.

34. Cabanac M. Pleasure: the common currency. J Theor Biol 155: 173-200, 1992.

35. Cabanac M. Heat Stress and Behavior. Compr Physiol 2011, Supplement 14: Handbook of Physiology, Environmental Physiology: 261-278. First published in print 1996.

36. Cadarette B, Levine L, Kolka M, Proulx G, Correa M, Sawka M. Heat strain reduction by ice-based and vapor compression liquid cooling systems with a toxic agent protective uniform. Aviat Sp Environ Med 73: 665-672, 2002

37. Cahusac PMB, Noyce R. A pharmacological study of slowly adapting mechanoreceptors responsive to cold thermal stimulation. Neuroscience 148: 489-500, 2007.

38. Cain W. Spatial discrimination of cutaneous warmth. Am J Psychol 86: 169-181, 1973.

39. Campero M, Baumann TK, Bostock H, Ochoa JL. Human cutaneous C fibres activated by cooling, heating and menthol. J Physiol 587: 5633-52, 2009.

40. Campero M, Serra J, Bostock H, Ochoa JL. Slowly conducting afferents activated by innocuous low temperature in human skin. J Physiol 535: 855-65, 2001.

41. Candas V, Libert J, Vogt J. Effect of hidromeiosis on sweat drippage during acclimation to humid heat. Eur J Appl Physiol 133: 123-133, 1980. 
42. Caterina MJ, Leffler A, Malmberg AB, Martin WJ, Trafton J, Petersen-Zeitz KR, Koltzenburg M, Basbaum Al, Julius D. Impaired nociception and pain sensation in mice lacking the capsaicin receptor. Science 288: 306-313, 2000.

43. Caterina MJ, Rosen TA, Tominaga M, Brake AJ, Julius D. A capsaicin-receptor homologue with a high threshold for noxious heat. Nature 398: 436-441, 1999.

44. Caterina MJ, Schumacher MA, Tominaga M, Rosen TA, Levine JD, Julius D. The capsaicin receptor: a heat-activated ion channel in the pain pathway. Nature 389: 816824, 1997.

45. Chatt $A$, Kenshalo $D$. Cerebral evoked responses to skin warming recorded from human scalp. Exp brain Res 455: 449-455, 1977.

46. Chen J, Kang D, Xu J, Lake M, Hogan JO, Sun C, Walter K, Yao B, Kim D. Species differences and molecular determinant of TRPA1 cold sensitivity. Nat Commun 4: 2501, 2013.

47. Chen J. The evolutionary divergence of TRPA1 channel: heat-sensitive, cold-sensitive and temperature-insensitive. Temperature 2: 158-159, 2015.

48. Cheng Y, Niu J, Gao N. Thermal comfort models: A review and numerical investigation. Build Environ 47: 13-22, 2012.

49. Christensen BN, Perl ER. Spinal neurons specifically excited by noxious or thermal stimuli: marginal zone of the dorsal horn. J Neurophysiol 33: 293-307, 1970.

50. Chung MK, Lee H, Caterina MJ. Warm temperatures activate TRPV4 in mouse 308 keratinocytes. J Biol Chem 278: 32037-32046, 2003.

51. Chung MK, Lee H, Mizuno A, Suzuki M, Caterina MJ. TRPV3 and TRPV4 mediate warmth-evoked currents in primary mouse keratinocytes. J Biol Chem 279: 21569 21575, 2004.

52. Clapham DE, Miller C. A thermodynamic framework for understanding temperature sensing by transient receptor potential (TRP) channels. Proc Natl Acad Sci U S A 108: 19492-19497, 2011.

53. Clark R, Edholm O. Man and his thermal environment. London : E. Arnold, 1985.

54. Colburn RW, Lubin M Lou, Stone DJ, Wang Y, Lawrence D, D'Andrea MR, Brandt MR, Liu Y, Flores CM, Qin N. Attenuated cold sensitivity in TRPM8 null mice. Neuron 54: 379386, 2007.

55. Cosens DJ, Manning A. Abnormal electroretinogram from a Drosophila mutant. Nature 224: 285-287, 1969.

56. Cotter J, Taylor N. The distribution of cutaneous sudomotor and alliesthesial thermosensitivity in mildly heat-stressed humans: an open-loop approach. J Physiol 565: 335-345, 2005.

57. Craig AD. How do you feel--now? The anterior insula and human awareness. Nat Rev Neurosci 10: 59-70, 2009.

58. Craig AD. Topographically organized projection to posterior insular cortex from the posterior portion of the ventral medial nucleus in the long-tailed macaque monkey. $J$ Comp Neurol 522: 36-63, 2014.

59. Craig AD. Significance of the insula for the evolution of human awareness of feelings from the body. Ann N Y Acad Sci 1225: 72-82, 2011.

60. Craig $A D$, Bushnell $M$, Zhang $E$, Blomqvist $A$. A thalamic nucleus specific for pain and temperature sensation. Nature 372: 770-773, 1994.

61. Craig $A D$, Bushnell $M$. The thermal grill illusion: unmasking the burn of cold pain. Science 265: 252-255, 1994.

62. Craig AD, Chen K, Bandy D, Reiman EM. Thermosensory activation of insular cortex. Nat Neurosci 3: 184-190, 2000.

63. Craig AD, Dostrovsky JO. Thermoreceptive lamina I trigeminothalamic neurons project to the nucleus submedius in the cat. Exp Brain Res 85: 470-474, 1991.

64. Craig AD, Dostrovsky JO. Differential projections of thermoreceptive and nociceptive lamina I trigeminothalamic and spinothalamic neurons in the cat. J Neurophysiol 86: 856870, 2001. 
65. Craig AD, Krout K, Andrew D. Quantitative response characteristics of thermoreceptive and nociceptive lamina I spinothalamic neurons in the cat. J Neurophysiol 86: 1459-1480, 2001.

66. Craig AD, Reiman E, Evans A, Bushnell M. Functional imaging of an illusion of pain. Nature 21: 258-260, 1996.

67. Craig $A D$. How do you feel? Interoception: the sense of the physiological condition of the body. Nat Rev Neurosci 3: 655-666, 2002.

68. Craig AD. Interoception: the sense of the physiological condition of the body. Curr Opin Neurobiol 13: 500-505, 2003.

69. Cramer MN, Jay O. Selecting the correct exercise intensity for unbiased comparisons of thermoregulatory responses between groups of different mass and surface area. $\mathrm{J}$ Appl Physiol 116: 1123-32, 2014.

70. Cramer MN, Jay O. Explained variance in the thermoregulatory responses to exercise: The independent roles of biophysical and fitness/fatness-related factors. J Appl Physiol DOI: 10.1152/japplphysiol.00281.2015, 2015.

71. Daanen HAM. Method and system for alerting the occurrence of wetness. EP Pat. 2,110,108, 2009.

72. Damasio A, Damasio H, Tranel D. Persistence of Feelings and Sentience after Bilateral Damage of the Insula. Cereb Cortex 23: 833-846, 2013.

73. Damasio AR. The Somatic Marker Hypothesis and the Possible Functions of the Prefrontal Cortex. Philos Trans R Soc B Biol Sci 351: 1413-1420, 1996.

74. Darian-Smith I, Johnson K, LaMotte $C$, Shigenaga $Y$, Kenins P, Champness P. Warm fibers innervating palmar and digital skin of the monkey: responses to thermal stimuli. $J$ Neurophysiol 42: 1297-1315, 1979.

75. Darian-Smith I, Johnson KO, Dykes R. "Cold" fiber population innervating palmar and digital skin of the monkey: responses to cooling pulses. J Neurophysiol 36: 325-346, 1973.

76. Darian-Smith I. Thermal Sensibility. Compr Physiol 2011, Supplement 3: Handbook of Physiology, The Nervous System, Sensory Processes: 879-913. First published in print 1984. doi: 10.1002/cphy.cp010319

77. Davis JB, Gray J, Gunthorpe MJ, Hatcher JP, Davey PT, Overend P, Harries MH, Latcham J, Clapham C, Atkinson K, Hughes SA, Rance K, Grau E, Harper AJ, Pugh PL, Rogers DC, Bingham S, Randall A, Sheardown SA.. Vanilloid receptor-1 is essential for inflammatory thermal hyperalgesia. Nature 405: 183-187, 2000.

78. Davis KD, Kwan CL, Crawley AP, Mikulis DJ. Functional MRI study of thalamic and cortical activations evoked by cutaneous heat, cold, and tactile stimuli. J Neurophysiol 80: 1533-1546, 1998.

79. Davis KD, Kwan CL, Crawley AP, Mikulis DJ. Functional MRI study of thalamic and cortical activations evoked by cutaneous heat, cold, and tactile stimuli. J Neurophysiol 80 : 1533-1546, 1998.

80. Davis KD, Lozano RM, Manduch M, Tasker RR, Kiss ZH, Dostrovsky JO. Thalamic relay site for cold perception in humans. J Neurophysiol 81: 1970-1973, 1999.

81. Davis KD. Cold-induced pain and prickle in the glabrous and hairy skin. Pain 75: 47-57, 1998.

82. Davis SL, Wilson TE, White AT, Frohman EM. Thermoregulation in multiple sclerosis. $J$ Appl Physiol 109: 1531-1537, 2010.

83. de Dear R. Revisiting an old hypothesis of human thermal perception: alliesthesia. Build Res Inf 39: 108-117, 2011.

84. Defrin R, Petrini L, Arendt-Nielsen L. Spatial summation of thermal sensations depends on skin type and skin sensitivity. Exp brain Res 198: 29-36, 2009.

85. Dhaka A, Earley TJ, Watson J, Patapoutian A. Visualizing cold spots: TRPM8-expressing sensory neurons and their projections. J Neurosci 28: 566-575, 2008.

86. Dhaka A, Murray AN, Mathur J, Earley TJ, Petrus MJ, Patapoutian A. TRPM8 is required for cold sensation in mice. Neuron 54: 371-378, 2007.

87. Donaldson HH. On the temperature-sense. Mind 10: 398-416, 1885. 
88. Dostrovsky JO, Craig AD. Cooling-specific spinothalamic neurons in the monkey. $J$ Neurophysiol 76: 3656-3665, 1996.

89. Driver J, Spence C. Multisensory perception: Beyond modularity and convergence. Curr Biol 10: R731-R735, 2000.

90. Duclaux R, Kenshalo DR. The temperature sensitivity of the type I slowly adapting mechanoreceptors in cats and monkeys. J Physiol 224: 647-664, 1972.

91. Duncan G, Bushnell M, Oliveras J, Bastrash N, Tremblay N. Thalamic VPM nucleus in the behaving monkey. III. Effects of reversible inactivation by lidocaine on thermal and mechanical discrimination. J Neurophysiol 70: 2086-2096, 1993.

92. Egan GF, Johnson J, Farrell M, McAllen R, Zamarripa F, McKinley MJ, Lancaster J, Denton D, Fox PT. Cortical, thalamic, and hypothalamic responses to cooling and warming the skin in awake humans: a positron-emission tomography study. Proc Natl Acad Sci U S A 102: 5262-5267, 2005.

93. Ehling $\mathrm{P}$, Cerina M, Budde T, Meuth SG, Bittner S. The CNS under pathophysiologic attack-examining the role of K2P channels. Pflügers Arch - Eur J Physiol 467: 959-972, 2015.

94. Ernst MO, Banks MS. Humans integrate visual and haptic information in a statistically optimal fashion. Nature 415: 429-433, 2002.

95. Fajardo O, Meseguer V, Belmonte C, Viana F. TRPA1 channels mediate cold temperature sensing in mammalian vagal sensory neurons: pharmacological and genetic evidence. J Neurosci 28: 7863-7875, 2008.

96. Farrell MJ, Trevaks D, Taylor $\mathrm{N}$ a S, McAllen RM. Brain stem representation of thermal and psychogenic sweating in humans. Am J Physiol Regul Integr Comp Physiol 304: R810-7, 2013.

97. Feketa $\vee V$, Marrelli SP. Induction of therapeutic hypothermia by pharmacological modulation of temperature-sensitive TRP channels : theoretical framework and practical considerations. Temperature 2: 244-257, 2015.

98. Filingeri D, Fournet D, Hodder S, Havenith $G$. Why wet feels wet? A neurophysiological model of human cutaneous wetness sensitivity. J Neurophysiol 112: 1457-1469, 2014.

99. Filingeri D, Fournet D, Hodder S, Havenith G. Body mapping of cutaneous wetness perception across the human torso during thermo-neutral and warm environmental exposures. J Appl Physiol 117: 887-897, 2014.

100. Filingeri $D$, Fournet $D$, Hodder $S$, Havenith $G$. Tactile cues significantly modulate the perception of sweat-induced skin wetness independently of the level of physical skin wetness. J Neurophysiol 113: 3462-3473, 2015.

101. Filingeri D, Havenith G. Human skin wetness perception: Psychophysical and neurophysiological bases. Temperature 2: 86-104, 2015.

102. Filingeri D, Redortier B, Hodder S, Havenith $G$. The role of decreasing contact temperatures and skin cooling in the perception of skin wetness. Neurosci Lett 551: 6569, 2013.

103. Filingeri D, Redortier B, Hodder S, Havenith G. Thermal and tactile interactions in the perception of local skin wetness at rest and during exercise in thermo-neutral and warm environments. Neuroscience 258: 121-130, 2014.

104. Filingeri D, Redortier B, Hodder S, Havenith G. Warm temperature stimulus suppresses the perception of skin wetness during initial contact with a wet surface. Ski Res Technol 21: 9-14, 2015.

105. Filingeri D. Humidity sensation, cockroaches, worms and humans: are common sensory mechanisms for hygrosensation shared across species? J Neurophysiol 114: 763-767, 2015.

106. Flouris AD. Functional architecture of behavioural thermoregulation. Eur J Appl Physiol 111: 1-8, 2011.

107. Fowler CJ, Sitzoglou K, Ali Z, Halonen P. The conduction velocities of peripheral nerve fibres conveying sensations of warming and cooling. J Neurol Neurosurg Psychiatry 51: 1164-1170, 1988. 
108. Frank SM, Raja SN, Bulcao CF, Goldstein DS. Relative contribution of core and cutaneous temperatures to thermal comfort and autonomic responses in humans. $\mathrm{J}$ Appl Physiol 86: 1588-1593, 1999.

109. Fukazawa T, Havenith G. Differences in comfort perception in relation to local and whole body skin wettedness. Eur J Appl Physiol 106: 15-24, 2009.

110. Gagge AP, Gonzalez RR. Mechanisms of Heat Exchange: Biophysics and Physiology. Compr Physiol 2011, Supplement 14: Handbook of Physiology, Environmental Physiology: 45-84. First published in print 1996. doi: 10.1002/cphy.cp040104.

111. Gagge AP, Stolwijk J, Hardy J. Comfort and thermal sensations and associated physiological responses at various ambient temperatures. Environ Res 1: 1-20, 1967.

112. Gagge AP. A new physiological variable associated with sensible and insensible perspiration. Am J Physiol - Legacy Content, 1937.

113. Gagnon D, Jay O, Kenny G. The evaporative requirement for heat balance determines whole-body sweat rate during exercise under conditions permitting full evaporation. $J$ Physiol 591(Pt 11): 2925-2935, 2013.

114. Gallio M, Ofstad TA, Macpherson LJ, Wang JW, Zuker CS. The coding of temperature in the Drosophila brain. Cell 144: 614-624, 2011.

115. Gavva NR, Bannon AW, Surapaneni S, Hovland DN, Lehto SG, Gore A, Juan T, Deng H, Han B, Klionsky L, Kuang R, Le A, Tamir R, Wang J, Youngblood B, Zhu D, Norman MH, Magal E, Treanor JJS, Louis J-C. The vanilloid receptor TRPV1 is tonically activated in vivo and involved in body temperature regulation. J Neurosci 27: 3366-3374, 2007.

116. Gavva NR, Treanor JJS, Garami A, Fang L, Surapaneni S, Akrami A, Alvarez F, Bak A, Darling M, Gore A, Jang GR, Kesslak JP, Ni L, Norman MH, Palluconi G, Rose MJ, Salfi M, Tan E, Romanovsky A a, Banfield C, Davar G. Pharmacological blockade of the vanilloid receptor TRPV1 elicits marked hyperthermia in humans. Pain 136: 202-210, 2008.

117. Gees M, Owsianik G, Nilius B, Voets T. TRP channels. Compr Physiol 2: 563-608, 2012.

118. Geisler WS, Kersten D. Illusions, perception and Bayes. Nat Neurosci 5: 508-510, 2002.

119. Gerrett N, Ouzzahra Y, Coleby S, Hobbs S, Redortier B, Voelcker T, Havenith G. Thermal sensitivity to warmth during rest and exercise: a sex comparison. Eur J Appl Physiol 114: 1451-1462, 2014.

120. Gerrett N, Redortier B, Voelcker T, Havenith G. A comparison of galvanic skin conductance and skin wettedness as indicators of thermal discomfort during moderate and high metabolic rates. J Therm Biol 38: 530-538, 2013.

121. Goldscheider A. Gesammelte Abhandlungen. Leipzig: Barth, 1898.

122. Graven-Nielsen T, Arendt-Nielsen L, Mense S. Thermosensitivity of muscle: highintensity thermal stimulation of muscle tissue induces muscle pain in humans. J Physiol 540: 647-656, 2002.

123. Green B. Localization of thermal sensation: An illusion and synthetic heat. Percept Psychophys 22: 331-337, 1977.

124. Green B. Temperature perception on the hand during static versus dynamic contact with a surface. Attention, Perception, Psychophys 71: 1185-1196, 2009.

125. Green BG, Pope JV. Innocuous cooling can produce nociceptive sensations that are inhibited during dynamic mechanical contact. Exp Brain Res 148: 290-299, 2003.

126. Green BG, Schoen KL. Evidence that tactile stimulation inhibits nociceptive sensations produced by innocuous contact cooling. Behav Brain Res 162: 90-98, 2005.

127. Green BG, Schoen KL. Thermal and nociceptive sensations from menthol and their suppression by dynamic contact. Behav Brain Res 176: 284-291, 2007.

128. Green BG. Temperature perception and nociception. J Neurobiol 61: 13-29, 2004.

129. Greenspan JD, Ohara S, Franaszczuk P, Veldhuijzen DS, Lenz FA. Cold stimuli evoke potentials that can be recorded directly from parasylvian cortex in humans. $J$ Neurophysiol 100: 2282-2286, 2008.

130. Greenspan JD, Roy EA, Caldwell PA, Farooq NS. Thermosensory intensity and affect throughout the perceptible range. Somatosens Mot Res 20: 19-26, 2003.

131. Güler AD, Lee H, lida T, Shimizu I, Tominaga M, Caterina M. Heat-evoked activation of the ion channel, TRPV4. J Neurosci 22: 6408-6414, 2002. 
132. Hadjimichael O, Kerns RD, Rizzo MA, Cutter G, Vollmer T. Persistent pain and uncomfortable sensations in persons with multiple sclerosis. Pain 127: 35-41, 2007.

133. Haggard P, lannetti GD, Longo MR. Spatial sensory organization and body representation in pain perception. Curr Biol 23: R164-176, 2013.

134. Hallin $\mathrm{R}$, Torebjörk $\mathrm{H}$, Wiesenfeld $\mathrm{Z}$. Nociceptors and warm receptors innervated by $\mathrm{C}$ fibres in human skin. J Neurol Neurosurg Psychiatry 45: 313-319, 1982.

135. Han ZS, Zhang ET, Craig AD. Nociceptive and thermoreceptive lamina I neurons are anatomically distinct. Nat Neurosci 1: 218-225, 1998.

136. Hansen $\mathrm{C}$, Hopf $\mathrm{H}$, Treede R. Paradoxical heat sensation in patients with multiple sclerosis Evidence for a supraspinal integration of temperature sensation. Brain 119: 1729-1736, 1996.

137. Hardy JD, Oppel TW. Studies in temperature sensation. III. The sensitivity of the body to heat and the spatial summation of the end organ responses. J Clin Invest 16: 533-540, 1937.

138. Hardy JD, Oppel TW. Studies in temperature sensation. IV. The stimulation of cold sensation by radiation. J Clin Invest 17: 771-778, 1938.

139. Havenith $\mathrm{G}$. The relative influence of physical fitness, acclimatization state, anthropometric measures and gender on individual reactions to heat stress. Eur J Appl Physiol 61: 419-427, 1990.

140. Havenith $\mathrm{G}$. Human surface to mass ratio and body core temperature in exercise heat stress-a concept revisited. J Therm Biol 26: 387-393, 2001.

141. Havenith G. Interaction of Clothing and Thermoregulation. Exog Dermatology 1: 221230, 2002.

142. Held K, Voets T, Vriens J. TRPM3 in temperature sensing and beyond. Temperature 2: 201-213, 2015.

143. Hellon R, Misra N, Provins K. Neurones in the somatosensory cortex of the rat responding to scrotal skin temperature changes. J Physiol 232: 401-411, 1973.

144. Hellon R. Thermoreceptors. Compr Physiol 2011, Supplement 8: Handbook of Physiology, The Cardiovascular System, Peripheral Circulation and Organ Blood Flow: 659-673. First published in print 1983.

145. Hensel $\mathrm{H}$, Andres $\mathrm{KH}$, von Düring M. Structure and function of cold receptors. Pflügers Arch 352: 1-10, 1974.

146. Hensel $\mathrm{H}$, Boman $\mathrm{K}$. Afferent impulses in cutaneous sensory nerves in human subjects. $\mathrm{J}$ Neurophysiol 23: 564-578, 1960.

147. Hensel H, Iggo A. Analysis of cutaneous warm and cold fibres in primates. Pflügers Arch 329: 1-8, 1971.

148. Hensel H, Kenshalo DR. Warm receptors in the nasal region of cats. J Physiol 204: 99112, 1969.

149. Hensel $\mathrm{H}$, Zotterman $\mathrm{Y}$. The response of mechanoreceptors to thermal stimulation. $\mathrm{J}$ Physiol 115: 16-24, 1951.

150. Hensel H. Neural processes in thermoregulation. [Online]. Physiol Rev 53: 948-1017, 1973.

151. Hensel $\mathrm{H}$. Thermoreception and temperature regulation. Monogr Physiol Soc Issue 38: 1321. Academic Press, 1981.

152. Hirata $\mathrm{H}$, Oshinsky ML. Ocular dryness excites two classes of corneal afferent neurons implicated in basal tearing in rats: involvement of transient receptor potential channels. $J$ Neurophysiol 107: 1199-1209, 2012.

153. Hirata $\mathrm{H}$, Oshinsky ML. Ocular dryness excites two classes of corneal afferent neurons implicated in basal tearing in rats: involvement of transient receptor potential channels. $J$ Neurophysiol 107: 1199-1209, 2012.

154. Ho HN, Watanabe J, Ando H, Kashino M. Somatotopic or spatiotopic? Frame of reference for localizing thermal sensations under thermo-tactile interactions. Atten Percept Psychophys 72: 1666-1675, 2010.

155. Ho HN, Watanabe J, Ando H, Kashino M. Mechanisms underlying referral of thermal sensations to sites of tactile stimulation. J Neurosci 31: 208-213, 2011. 
156. van Hoof J. Forty years of Fanger's model of thermal comfort: comfort for all? Indoor Air 18: 182-201, 2008.

157. Hua LH, Strigo IA, Baxter LC, Johnson SC, Craig AD. Anteroposterior somatotopy of innocuous cooling activation focus in human dorsal posterior insular cortex. Am J Physiol Regul Integr Comp Physiol 289: R319-R325, 2005.

158. Huang SM, Li X, Yu Y, Wang J, Caterina MJ. TRPV3 and TRPV4 ion channels are not major contributors to mouse heat sensation. Mol Pain 7: 37, 2011.

159. Iannetti GD, Truini a, Romaniello a, Galeotti F, Rizzo C, Manfredi M, Cruccu G. Evidence of a specific spinal pathway for the sense of warmth in humans. J Neurophysiol 89: 562570, 2003.

160. lannetti GD, Zambreanu L, Tracey I. Similar nociceptive afferents mediate psychophysical and electrophysiological responses to heat stimulation of glabrous and hairy skin in humans. J Physiol 577: 235-248, 2006.

161. Iannetti GD, Zambreanu L, Tracey I. Similar nociceptive afferents mediate psychophysical and electrophysiological responses to heat stimulation of glabrous and hairy skin in humans. J Physiol 577: 235-48, 2006.

162. Iggo A. Cutaneous thermoreceptors in primates and sub-primates. J Physiol 200: 403430, 1969.

163. Jessen C. Interaction of Body Temperatures in Control of Thermoregulatory Effector Mechanisms. Compr Physiol 2011, Supplement 14: Handbook of Physiology, Environmental Physiology: 127-138. First published in print 1996.

164. Johnson JM, Minson CT, Kellogg DL. Cutaneous vasodilator and vasoconstrictor mechanisms in temperature regulation. Compr Physiol 4: 33-89, 2014.

165. Johnson KO, Darian-Smith I. Coding of incremental changes in skin temperature by a population of warm fibers in the monkey: correlation with intensity discrimination in man. $J$ Neurophysiol 42: 1332-1353, 1979.

166. Johnson KO, Darian-Smith I, LaMotte C. Peripheral neural determinants of temperature discrimination in man: a correlative study of responses to cooling skin. $J$ Neurophysiol 36: 347-370, 1973.

167. Jordt SE, Bautista DM, Chuang HH, McKemy DD, Zygmunt PM, Högestätt ED, Meng ID, Julius D. Mustard oils and cannabinoids excite sensory nerve fibres through the TRP channel ANKTM1. Nature 427: 260-265, 2004.

168. Kakigi R, Shibasaki H. Estimation of conduction velocity of the spino-thalamic tract in man. Electroencephalogr Clin Neurophysiol 80: 39-45, 1991.

169. Kandel ER, Schwartz JH, Jessell TM. Principles of neural sciences. USA ET - 4th: McGraw-Hill, 2000.

170. Kang D, Choe C, Kim D. Thermosensitivity of the two-pore domain K+ channels TREK-2 and TRAAK. J Physiol 564: 103-116, 2005.

171. Karashima Y1, Talavera K, Everaerts W, Janssens A, Kwan KY, Vennekens R, Nilius B, Voets T. TRPA1 acts as a cold sensor in vitro and in vivo. Proc Natl Acad Sci U S A 106: 1273-1278, 2009.

172. Kenney W, Munce T. Invited review: aging and human temperature regulation. J Appl Physiol 95: 2598-2603, 2003.

173. Kenshalo DR, Decker T, Hamilton A. Spatial summation on the forehead, forearm, and back produced by radiant and conducted heat. J Comp Physiol Psychol 63: 510-515, 1967.

174. Kenshalo DR, Duclaux R. Response characteristics of cutaneous cold receptors in the monkey. J Neurophysiol 40: 319-332, 1977.

175. Kenshalo DR, Holmes C, Wood P. Warm and cool thresholds as a function of rate of stimulus temperature change. Percept Psychophys 3: 81-84, 1968.

176. Kenshalo DR, Nafe JP, Brooks B. Variations in thermal sensitivity. Science 134: 105-105, 1961.

177. Kenshalo DR, Scott HA Jr. Temporal course of thermal adaptation. Science 151: 10951096, 1966. 
178. Kim J, Lee M, Shim HJ, Ghaffari R, Cho HR, Son D, Jung YH, Soh M, Choi C, Jung S, Chu K, Jeon D, Lee S-T, Kim JH, Choi SH, Hyeon T, Kim D-H. Stretchable silicon nanoribbon electronics for skin prosthesis. Nat Commun 5: 5747, 2014.

179. Kim JH, Greenspan JD, Coghill RC, Ohara S, Lenz F a. Lesions limited to the human thalamic principal somatosensory nucleus (ventral caudal) are associated with loss of cold sensations and central pain. J Neurosci 27: 4995-5004, 2007.

180. Kingma BRM, Frijns AJH, Schellen L, Lichtenbelt WDVM. Beyond the classic thermoneutral zone: Including thermal comfort. Temperature 1: 142-149, 2014.

181. Knill D, Richards W. Perception as Bayesian inference. Cambridge Univeristy Press. New York, NY, USA: 1996.

182. Knowlton WM, Bifolck-Fisher A, Bautista DM, McKemy DD. TRPM8, but not TRPA1, is required for neural and behavioral responses to acute noxious cold temperatures and cold-mimetics in vivo. Pain 150: 340-350, 2010.

183. Knowlton WM, Palkar R, Lippoldt EK, McCoy DD, Baluch F, Chen J, McKemy DD. A sensory-labeled line for cold: TRPM8-expressing sensory neurons define the cellular basis for cold, cold pain, and cooling-mediated analgesia. J Neurosci 33: 2837-2848, 2013.

184. Konietzny F, Hensel H. Warm fiber activity in human skin nerves. Pflügers Arch Eur J Physiol 267: 265-267, 1975.

185. Kuntz A, Haselwood LA. Circulatory reactions in the gastrointestinal tract elicited by localized cutaneous stimulation. Am Heart J 20: 743-749, 1940.

186. Kwan CL, Crawley AP, Mikulis DJ, Davis KD. An fMRI study of the anterior cingulate cortex and surrounding medial wall activations evoked by noxious cutaneous heat and cold stimuli. Pain 85: 359-374, 2000.

187. LaMotte RH, Campbell JN. Comparison of responses of warm and nociceptive C-fiber afferents in monkey with human judgments of thermal pain. J Neurophysiol 41: 509-528, 1978.

188. Landgren S. Cortical reception of cold impulses from the tongue of the cat. Acta Physiol Scand 40: 202-209, 1957.

189. Laursen WJ, Anderson EO, Hoffstaetter LJ, Bagriantsev SN, Laursen WJ, Anderson EO, Hoffstaetter LJ, Bagriantsev SN, Gracheva EO. Species-specific temperature sensitivity of TRPA1. Temperature 2: 214-226, 2015.

190. Lee H, Lida T, Mizuno A, Suzuki M, Caterina MJ. Altered thermal selection behavior in mice lacking transient receptor potential vanilloid 4. J Neurosci 25: 1304-1310, 2005.

191. Lee J, Nakao K, Tochihara Y. Validity of perceived skin wettedness mapping to evaluate heat strain. Eur J Appl Physiol 111: 2581-2591, 2011.

192. Lee J, Ohara S. Pain and temperature encoding in the human thalamic somatic sensory nucleus (ventral caudal): inhibition-related bursting evoked by somatic stimuli. J Neurophysiol 94: 1676-1687, 2005.

193. Lenz FA, Dougherty PM. Neurons in the human thalamic somatosensory nucleus (Ventralis caudalis) respond to innocuous cool and mechanical stimuli. J Neurophysiol 79: 2227-2230, 1998.

194. Lenz FA, Kwan HC, Martin R, Tasker R, Richardson RT, Dostrovsky JO. Characteristics of somatotopic organization and spontaneous neuronal activity in the region of the thalamic principal sensory nucleus in patients with spinal cord. J Neurophysiol 72: 15701587, 1994.

195. Lenz FA1, Seike M, Richardson RT, Lin YC, Baker FH, Khoja I, Jaeger CJ, Gracely RH. Thermal and pain sensations evoked by microstimulation in the area of human ventrocaudal nucleus. J Neurophysiol 70: 200-212, 1993.

196. Li Y. Perceptions of temperature, moisture and comfort in clothing during environmental transients. Ergonomics 48: 234-248, 2005.

197. Liao M, Cao E, Julius D, Cheng Y. Structure of the TRPV1 ion channel determined by electron cryo-microscopy. Nature 504: 107-112, 2013.

198. Lindstedt F, Johansson B, Martinsen S, Kosek E, Fransson P, Ingvar M. Evidence for thalamic involvement in the thermal grill illusion: an FMRI study. PLoS One 6: e27075, 2011. 
199. Liu L, Li Y, Wang R, Yin C, Dong Q, Hing H, Kim C, Welsh MJ. Drosophila

hygrosensation requires the TRP channels water witch and nanchung. Nature 450: 294298, 2007.

200. Lochmann T, Deneve S. Neural processing as causal inference. Curr Opin Neurobiol 21: 774-781, 2011.

201. Long RR. Sensitivity of cutaneous cold fibers to noxious heat: paradoxical cold discharge. J Neurophysiol 40: 489-502, 1977.

202. Lumpkin EA, Caterina MJ. Mechanisms of sensory transduction in the skin. Nature 445: 858-865, 2007.

203. Mackenzie RA, Burke D, Skuse NF, Lethlean AK. Fibre function and perception during cutaneous nerve block. J Neurol Neurosurg Psychiatry 38: 865-873, 1975.

204. Mandadi S, Sokabe T, Shibasaki K, Katanosaka K, Mizuno A, Moqrich A, Patapoutian A, Fukumi-Tominaga T, Mizumura K, Tominaga M. TRPV3 in keratinocytes transmits temperature information to sensory neurons via ATP. Pflugers Arch 458: 1093-1102, 2009.

205. Marshall A, Ackerley R. P990: Uncovering the tactile aspects in sensing drops of water. Clin Neurophysiol 125: S311, 2014.

206. McGlone F, Reilly D. The cutaneous sensory system. Neurosci Biobehav Rev 34: 148159, 2010.

207. McGlone F, Wessberg J, Olausson H. Discriminative and Affective Touch: Sensing and Feeling. Neuron 82: 737-755, 2014.

208. McKemy DD, Neuhausser W, Julius D. Identification of a cold receptor reveals a general role for TRP channels in thermosensation. Nature 416: 52-58, 2002.

209. McKemy DD. How cold is it? TRPM8 and TRPA1 in the molecular logic of cold sensation. Mol Pain 1: 16, 2005.

210. McKemy DD. Temperature sensing across species. Pflugers Arch 454: 777-791, 2007.

211. Mekjavic I, Eiken O. Contribution of thermal and nonthermal factors to the regulation of body temperature in humans. J Appl Physiol 100: 2065-2072, 2006.

212. Mekjavic I, Sundberg C, Linnarsson D. Core temperature "null zone". J Appl Physiol 71: 1289-1295, 1991.

213. Milenkovic N, Zhao W-J, Walcher J, Albert T, Siemens J, Lewin GR, Poulet JFA. A somatosensory circuit for cooling perception in mice. Nat Neurosci 17: 1560-1566, 2014.

214. Millqvist E. TRP channels and temperature in airway disease-clinical significance. Temperature 2: 172-177, 2015.

215. Montell C, Rubin GM. Molecular characterization of the Drosophila trp locus: a putative integral membrane protein required for phototransduction. Neuron 2: 1313-1323, 1989.

216. Moqrich A, Hwang SW, Earley TJ, Petrus MJ, Murray AN, Spencer KS, Andahazy M, Story GM, Patapoutian A. Impaired thermosensation in mice lacking TRPV3, a heat and camphor sensor in the skin. Science 307: 1468-1472, 2005.

217. Morris NB, Bain AR, Cramer MN, Jay O. Evidence that transient changes in sudomotor output with cold and warm fluid ingestion are independently modulated by abdominal, but not oral thermoreceptors. J Appl Physiol (1985) 116: 1088-1095, 2014.

218. Morrison S, Nakamura K. Central neural pathways for thermoregulation. Front Biosci 16: 74-104, 2011.

219. Morrison S. Carl Ludwig Distinguished Lectureship of the APS Neural Control and Autonomic Regulation Section: Central neural pathways for thermoregulatory cold defense. J Appl Physiol 110: 1137-1149, 2011.

220. Morrison SF, Madden CJ. Central nervous system regulation of brown adipose tissue. Compr Physiol 4: 1677-713, 2014.

221. Morrison SF. The thermostat concept - significant for mechanical temperature control systems, but irrelevant to mammalian thermoregulatory networks. Temperature 2: 332 333, 2015.

222. Nadel ER, Mitchell JW, Stolwijk JA. Differential thermal sensitivity in the human skin. Pflugers Arch 340: 71-76, 1973.

223. Nadel ER, Stolwijk JA. Effect of skin wettedness on sweat gland response. J Appl Physiol 35: 689-694, 1973. 
224. Nakamura K, Morrison S. A thermosensory pathway that controls body temperature. Nat Neurosci 11: 62-71, 2007.

225. Nakamura K. Central circuitries for body temperature regulation and fever. Am J Physiol Regul Integr Comp Physiol 301: R1207-228, 2011.

226. Nakamura M, Yoda T, Crawshaw L, Yasuhara S, Saito Y, Kasuga M, Nagashima K, Kanosue K. Regional differences in temperature sensation and thermal comfort in humans. J Appl Physiol (1985) 105: 1897-1906, 2008.

227. Niedermann R, Rossi R. Objective and subjective evaluation of the human thermal sensation of wet fabrics. Text Res J 82: 374-384, 2012.

228. Noël J, Zimmermann K, Busserolles J, Deval E, Alloui A, Diochot S, Guy N, Borsotto M, Reeh $\mathrm{P}$, Eschalier A, Lazdunski M. The mechano-activated $\mathrm{K}+$ channels TRAAK and TREK-1 control both warm and cold perception. EMBO J 28: 1308-1318, 2009.

229. Norrsell U, Craig AD. Behavioral thermosensitivity after lesions of thalamic target areas of a thermosensory spinothalamic pathway in the cat. J Neurophysiol 82: 611-625, 1999.

230. Norrsell U, Finger S, Lajonchere C. Cutaneous sensory spots and the "law of specific nerve energies": history and development of ideas. Brain Res Bull 48: 457-65, 1999.

231. Norrsell $U$. Thermosensory defects after cervical spinal cord lesions in the cat. Exp Brain Res 494: 479-494, 1979.

232. Olausson H, Wessberg J, Morrison I, McGlone F, Vallbo A. The neurophysiology of unmyelinated tactile afferents. Neurosci Biobehav Rev 34: 185-191, 2010.

233. Olesen B, Brager G. A better way to predict comfort: The new ASHRAE standard 552004. ASHRAE Journal, 2004.

234. Oppel TW, Hardy JD. Studies in temperature sensation. I. A comparison of the sensation produced by infra-red and visible radiation. J Clin Invest 16: 517-524, 1937.

235. Oppel TW, Hardy JD. Studies in temperature sensation. II. The temperature changes responsible for the stimulation of the heat end organs. J Clin Invest 16: 525-531, 1937.

236. Osterberg A, Boivie J. Central pain in multiple sclerosis - sensory abnormalities. Eur J Pain 14: 104-110, 2010.

237. Ostrowsky K, Magnin M, Ryvlin P, Isnard J, Guenot M, Mauguière F. Representation of pain and somatic sensation in the human insula: a study of responses to direct electrical cortical stimulation. Cereb Cortex 12: 376-85, 2002.

238. Ouzzahra Y, Havenith G, Redortier B. Regional distribution of thermal sensitivity to cold at rest and during mild exercise in males. J Therm Biol 37: 517-523, 2012.

239. Park U, Vastani N, Guan Y, Raja SN, Koltzenburg M, Caterina MJ. TRP vanilloid 2 knockout mice are susceptible to perinatal lethality but display normal thermal and mechanical nociception. J Neurosci 31: 11425-1136, 2011.

240. Parra A, Madrid R, Echevarria D, del Olmo S, Morenilla-Palao C, Acosta MC, Gallar J, Dhaka A, Viana F, Belmonte $C$. Ocular surface wetness is regulated by TRPM8dependent cold thermoreceptors of the cornea. Nat Med 16: 1396-1399, 2010.

241. Parsons K. Human Thermal Environments. Third Ed. London, UK: CRC Press, Taylor \& Francis group, 2014.

242. Patapoutian A, Peier AM, Story GM, Viswanath V. ThermoTRP channels and beyond: mechanisms of temperature sensation. Nat Rev Neurosci 4: 529-539, 2003.

243. Peier AM, Mogrich A, Hergarden A. A TRP channel that senses cold stimuli and menthol. Cell 108: 705-715, 2002.

244. Peier AM, Reeve AJ, Andersson DA, Moqrich A, Earley TJ, Hergarden AC, Story GM, Colley S, Hogenesch JB, McIntyre P, Bevan S, Patapoutian A. A heat-sensitive TRP channel expressed in keratinocytes. Science 296: 2046-2049, 2002.

245. Pessoa L, Thompson E, Noe A. Finding out about filling-in: A guide to perceptual completion for visual science and the philosophy of perception. Behav Brain Sci 21: 723748, 1998.

246. Pleger B, Villringer A. The human somatosensory system: from perception to decision making. Prog Neurobiol 103: 76-97, 2013.

247. Pogorzala LA, Mishra SK, Hoon MA. The cellular code for mammalian thermosensation. J Neurosci 33: 5533-5541, 2013. 
248. Price D, Hayes R, Ruda M, Dubner R. Spatial and temporal transformations of input to spinothalamic tract neurons and their relation to somatic sensations. J Neurophysiol 41: 933-947, 1978.

249. Qiu Y, Inui K, Wang X, Tran T, Kakigi R. Conduction velocity of the spinothalamic tract in humans as assessed by CO 2 laser stimulation of C-fibers. Neurosci Lett 311: 181-184, 2001.

250. Renigunta $V$, Schlichthörl G, Daut J. Much more than a leak: structure and function of K2P-channels. Pflügers Arch Eur J Physiol 467: 867-894, 2015.

251. Rolls ET, Grabenhorst F, Parris BA. Warm pleasant feelings in the brain. Neuroimage 41: 1504-1513, 2008.

252. Rolls ET. The affective and cognitive processing of touch, oral texture, and temperature in the brain. Neurosci Biobehav Rev 34: 237-245, 2010.

253. Romanovsky AA. Skin temperature: its role in thermoregulation. Acta Physiol 210: 498507, 2014.

254. Romanovsky AA. Thermoregulation: some concepts have changed. Functional architecture of the thermoregulatory system. Am J Physiol Regul Integr Comp Physiol 292: 37-47, 2007.

255. Romanovsky AA, Almeida MC, Garami A, Steiner AA, Norman MH, Morrison SF, Nakamura K, Burmeister JJ, Nucci TB. The Transient Receptor Potential Vanilloid-1 Channel in Thermoregulation : A Thermosensor It Is Not. 61: 228-261, 2009.

256. Rushmer RF, Buettner KJK, Short JM, Odland GF. The skin. Science 154: 343-348, 1966.

257. Russell J, Vidal-Gadea AG, Makay A, Lanam C, Pierce-Shimomura JT. Humidity sensation requires both mechanosensory and thermosensory pathways in Caenorhabditis elegans. Proc Natl Acad Sci U S A 111: 8269-8274, 2014.

258. Saal HP, Bensmaia SJ. Touch is a team effort: interplay of submodalities in cutaneous sensibility. Trends Neurosci 37: 689-697, 2014.

259. Satinoff E. Behavioral Thermoregulation in the Cold. Compr Physiol 14: 481-505, 2011.

260. Sawamoto N, Honda M, Okada T, Hanakawa T, Kanda M, Fukuyama H, Konishi J, Shibasaki $H$. Expectation of pain enhances responses to nonpainful somatosensory stimulation in the anterior cingulate cortex and parietal operculum/posterior insula: an event-related functional magnetic resonance imaging study. J Neurosci 20: 7438-7445, 2000.

261. Schepers R, Ringkamp M. Thermoreceptors and thermosensitive afferents. Neurosci Biobehav Rev 34: 177-184, 2010.

262. Schlader Z, Stannard S, Mündel T. Human thermoregulatory behavior during rest and exercise - a prospective review. Physiol Behav 99: 269-275, 2010.

263. Segerdahl AR, Mezue M, Okell TW, Farrar JT, Tracey I. The dorsal posterior insula subserves a fundamental role in human pain. Nat Neurosci 18: 499-500, 2015.

264. Serra J, Campero M, Bostock H, Ochoa J. Two types of $\mathrm{C}$ nociceptors in human skin and their behavior in areas of capsaicin-induced secondary hyperalgesia. J Neurophysiol 91: 2770-2781, 2004.

265. Shelford V. A comparison of the responses of animals in gradient of environmental factors with particular reference to the method of reaction of representatives of the various groups from protozoa to mammals. Science 48: 225-230, 1918.

266. Shibasaki M, Crandall CG. Mechanisms and controllers of eccrine sweating in humans. Front Biosci (Schol Ed) 2: 685-696, 2010.

267. Shibasaki M, Sakai M, Oda M, Crandall CG. Muscle mechanoreceptor modulation of sweat rate during recovery from moderate exercise. J Appl Physiol 96: 2115-2119, 2004.

268. Simon E, Pierau F, Taylor D. Central and peripheral thermal control of effectors in homeothermic temperature regulation. Physiol Rev 66: 235-300, 1986.

269. Smith GD, Gunthorpe MJ, Kelsell RE, Hayes PD, Reilly P, Facer P, Wright JE, Jerman JC, Walhin JP, Ooi L, Egerton J, Charles KJ, Smart D, Randall AD, Anand P, Davis JB. TRPV3 is a temperature-sensitive vanilloid receptor-like protein. Nature 418: 186-190, 2002. 
270. Van Someren EJW, Raymann RJEM, Scherder EJA, Daanen HA M, Swaab DF. Circadian and age-related modulation of thermoreception and temperature regulation: mechanisms and functional implications. Ageing Res Rev 1: 721-778, 2002.

271. Spiller WG. Remarks on the central representation of sensation. J Nerv Ment Dis 42: 399418, 1915.

272. Spray DC. Cutaneous temperature receptors. Annu Rev Physiol 48: 625-638, 1986.

273. Stevens JC, Choo KK. Temperature sensitivity of the body surface over the life span. Somatosens Mot Res 15:13-28, 1998.

274. Stevens JC, Marks LE, Simonson DC. Regional sensitivity and spatial summation in the warmth sense. Physiol Behav 13: 825-836, 1974.

275. Stevens JC. Variation of cold sensitivity over the body surface. Sens Processes 3: 317326, 1979.

276. Stocks J, Taylor N, Tipton M, Greenleaf J. Human physiological responses to cold exposure. Aviat Space Environ Med 75: 444-57, 2004.

277. Stolwijk JAJ, Hardy JD. Control of body temperature. Compr Physiol 2011, Supplement 26: Handbook of Physiology, Reactions to Environmental Agents: 45-68. First published in print 1977.

278. Stookey B. Further light on the transmission of pain and temperature within the spinal cord: human cordotomy to abolish pain sense without destroying temperature sense. $J$ Nerv Ment Dis 69: 552-557, 1929.

279. Story G, Peier A, Reeve A, Eid S. ANKTM1, a TRP-like channel expressed in nociceptive neurons, is activated by cold temperatures. Cell 112: 819-829, 2003.

280. Strusberg I, Mendelberg RC, Serra H a, Strusberg AM. Influence of weather conditions on rheumatic pain. J Rheumatol 29: 335-338, 2002.

281. Sumowski JF, Leavitt VM. Body temperature is elevated and linked to fatigue in relapsing-remitting multiple sclerosis, even without heat exposure. Arch Phys Med Rehabil 95: 1298-1302, 2014.

282. Susser E, Sprecher E, Yarnitsky D. Paradoxical heat sensation in healthy subjects: peripherally conducted by A delta or C fibres? Brain 122: 239-246, 1999.

283. Svendsen KB, Jensen TS, Hansen HJ, Bach FW. Sensory function and quality of life in patients with multiple sclerosis and pain. Pain 114: 473-481, 2005.

284. Sweeney MM, Branson DH. Sensorial Comfort: Part I: A Psychophysical Method for Assessing Moisture Sensation in Clothing. Text Res J 60: 371-377, 1990.

285. Sweeney MM, Branson DH. Sensorial Comfort: Part II: A Magnitude Estimation Approach for Assessing Moisture Sensation 1. Text Res J 60: 447-452, 1990.

286. Taylor NA S. Human heat adaptation. Compr Physiol 4: 325-365, 2014.

287. Thunberg T. Förnimmelserne vid till samma ställe lokaliserad, samtidigt pågående köldoch värmeretning. Uppsala Läkfören Förh 1: 489-495, 1896.

288. Tichy $\mathrm{H}$, Kallina W. Insect hygroreceptor responses to continuous changes in humidity and air pressure. J Neurophysiol 103: 3274-3286, 2010.

289. Tominaga M, Caterina M. Thermosensation and pain. J Neurobio/ 61: 3-12, 2004.

290. Tommerdahl M, Favorov O V, Whitsel BL. Dynamic representations of the somatosensory cortex. Neurosci Biobehav Rev 34: 160-170, 2010.

291. Torebjörk HE, Hallin RG. Identification of afferent $C$ units in intact human skin nerves. Brain Res 67: 387-403, 1974.

292. Torebjörk HE, Hallin RG. Perceptual changes accompanying controlled preferential blocking of $A$ and $C$ fibre responses in intact human skin nerves. Exp Brain Res 16: 321332, 1973.

293. Tsunozaki M, Bautista DM. Mammalian somatosensory mechanotransduction. Curr Opin Neurobiol 19: 362-369, 2009.

294. Vallbo AB, Olausson H, Wessberg J, Kakuda N. Receptive field characteristics of tactile units with myelinated afferents in hairy skin of human subjects. J Physiol 483: 783-795, 1995.

295. Vanos J, Warland J, Gillespie T, Kenny N. Thermal comfort modelling of body temperature and psychological variations of a human exercising in an outdoor environment. Int J Biometeorol 56: 21-32, 2012. 
296. Veldhuijzen DS, Greenspan JD, Kim JH, Lenz FA. Altered pain and thermal sensation in subjects with isolated parietal and insular cortical lesions. Eur J Pain 14: 535.e1-11, 2010.

297. Viana F, de la Peña E, Belmonte C. Specificity of cold thermotransduction is determined by differential ionic channel expression. Nat Neurosci 5: 254-260, 2002.

298. Voets T, Droogmans G, Wissenbach U, Janssens A, Flockerzi V, Nilius B. The principle of temperature-dependent gating in cold- and heat-sensitive TRP channels. Nature 430: 748-754, 2004.

299. Vriens J, Nilius B, Voets T. Peripheral thermosensation in mammals [Online]. Nat Rev Neurosci 15: 573-589, 2014.

300. Vriens J, Owsianik G, Hofmann T, Philipp SE, Stab J, Chen X, Benoit M, Xue F, Janssens A, Kerselaers S, Oberwinkler J, Vennekens R, Gudermann T, Nilius B, Voets T. TRPM3 is a nociceptor channel involved in the detection of noxious heat. Neuron 70: 482-494, 2011.

301. Wang $\mathrm{H}$, Siemens J. TRP ion channels in thermosensation, thermoregulation and metabolism. Temperature 2: 178-187, 2015.

302. Watanabe H, Vriens J, Suh SH, Benham CD, Droogmans G, Nilius B. Heat-evoked activation of TRPV4 channels in a HEK293 cell expression system and in native mouse aorta endothelial cells. J Biol Chem 277: 47044-47051, 2002.

303. Weiss Y, Simoncelli EP, Adelson EH. Motion illusions as optimal percepts. Nat Neurosci 5: 598-604, 2002.

304. White J, Sweet W, Hawkins R, Nilges R. Anterolateral cordotomy: results, complications and causes of failure. Brain 73: 346-367, 1950.

305. Willis WD, Trevino DL, Coulter JD, Maunz RA. Responses of primate spinothalamic tract neurons to natural stimulation of hindlimb. J Neurophysiol 37: 358-372, 1974.

306. Willis WD. Cold, pain and the brain. Nature 373: 19-20, 1995.

307. Wingo JE, Low DA, Keller DM, Brothers RM, Shibasaki M, Crandall CG. Skin blood flow and local temperature independently modify sweat rate during passive heat stress in humans. J Appl Physiol 109: 1301-1306, 2010.

308. Xu H, Ramsey I, Kotecha S, Moran M. TRPV3 is a calcium-permeable temperaturesensitive cation channel. Nature 418: 181-186, 2002.

309. Yarnitsky D, Ochoa JL. Warm and cold specific somatosensory systems Psychophysical thresholds, reaction times and peripheral conduction velocities. Brain 114: 1819-1826, 1991.

310. Yarnitsky D, Ochoa JL. Release of cold-induced burning pain by block of cold-specific afferent input. Brain 113: 893-902, 1990.

311. Zeisberger E, Roth J. Central Regulation of Adaptive Responses to Heat and Cold. Compr Physiol 2011, Supplement 14: Handbook of Physiology, Environmental Physiology: 579-595. First published in print 1996.

312. Zimmermann K, Leffler A, Babes A, Cendan CM, Carr RW, Kobayashi J, Nau C, Wood JN, Reeh PW. Sensory neuron sodium channel Nav1.8 is essential for pain at low temperatures. Nature 447: 855-858, 2007.

313. Zotterman Y. Action potentials in the glossopharyngeal nerve and in the chorda tympani. Skand Arch Physiol 72: 73-77, 1935.

314. Zotterman Y. Specific action potentials in the lingual nerve of cat. Skand Arch Physiol 75: 105-119, 1936.

315. Zotterman Y. Thermal sensations. In: Handbook of Physiology, Section I, Neurophysiology, Volume I. 1959, p. 431-458.

\section{Tables}

Table 1. Regional distribution of cold and warm spots per square centimeter of human skin [adapted from Hensel (126), p. 29]. 


\begin{tabular}{lcc} 
Skin region & Cold spots per $\mathbf{c m}^{2}$ & Warm spots per $\mathbf{c m}^{2}$ \\
\hline Forehead & $5.5-8.0$ & 1.0 \\
Nose & 8.0 & \\
Lips & $16.0-19.0$ & 1.7 \\
Other parts of face & $8.5-9.0$ & 0.3 \\
Chest & $9.0-10.2$ & \\
Abdomen & $8.0-12.5$ & \\
Back & 7.8 & $0.3-0.4$ \\
Upper arm & $5.0-6.5$ & 0.5 \\
Forearm & $6.0-7.5$ & \\
Back of hand & 7.4 & 1.7 \\
Palm of hand & $1.0-5.0$ & \\
Finger dorsal & $7.0-9.0$ & \\
Finger volar & $2.0-4.0$ & \\
Thigh & $4.5-5.2$ & \\
Calf & $4.3-5.7$ & \\
Back of foot & 5.6 & \\
Sole of foot & 3.4 & \\
\hline & & \\
\hline
\end{tabular}

\section{Figure Legends}

\section{Link to figures:}

http://www.comprehensivephysiology.com/WileyCDA/CompPhysArticle/refld-c150040.html

Figure 1. Effects of absolute skin temperature and rate of change in skin temperature on thermal sensations. Panel A shows changes in the absolute temperature threshold (i.e. the relative change in skin temperature required to induce a thermal sensation; solid squares, solid curves) and in the just noticeable difference threshold (open squares, broken curves), resulting from different adapting skin temperatures (curve 1: males; curve 2: females). It can be observed that, when detecting decreases in skin temperature, the warmer the baseline temperature, the greater the change in temperature required to induce a cold sensation. A similar effect is noticeable when detecting increases in skin temperature [From Kenshalo et al. (176); reprinted with permission from AAAS]. Panel $B$ shows the effect or the rate or the stimulus temperature change upon the 
warm and cool thresholds of three male subjects measured at normal skin temperature. The ordinate shows the change from normal skin temperature necessary to produce threshold warm and cool sensations at each or the rates or change in temperature. Rates or change as low as 0.1 ${ }^{\circ} \mathrm{C} / \mathrm{sec}$ railed to increase either threshold. Rates or temperature change slower than $0.1^{\circ} \mathrm{C} / \mathrm{sec}$ resulted in increases in both warm and cool thresholds although its effect was more pronounced upon the warm threshold [From Kenshalo et al. (175); with kind permission from Springer Science and Business Media]. Panel $C$ shows the relationship between the intensity of cooling pulses (duration: 4s) applied to the hand and related subjective magnitude estimates of thermal sensation. Skin had been previously adapted at $34^{\circ} \mathrm{C}$ [From Darian-Smith (76); $\odot$ The American Physiological Society].

Figure 2. Relationships between intensity of radiant heating applied to the (A) cheek, (B) forearm, (C) calf and (D) back and subjective magnitude estimates of thermal sensation, as resulting from stimulation of different areal extents [Reprinted from Stevens et al. (274) with permission from Elsevier].

Figure 3. Body maps showing regional distribution of $(A)$ skin cooling $\left({ }^{\circ} \mathrm{C}\right),(B)$ absolute mean votes for thermal sensation and $(C)$ frequency of wetness perception, as resulting from $10-s$ application of a relative cold-dry stimulus $\left(15^{\circ} \mathrm{C}\right.$ lower than local skin temperature) to each of 12 skin sites (i.e. 6 on the front and 6 on the back torso). Data were collected on the left side of the body and the body maps presented were developed assuming left-right symmetry. Regions showing greater skin cooling, colder sensations and more frequent wetness perceptions are represented in darker colors. The rating scales used by the participants to score their absolute thermal and pleasantness sensations are reported next to the respective body maps. Two main tendencies are shown. First, the regional differences in thermal and wetness sensations present a similar pattern across the torso (e.g. as opposed to the chest, the lateral and lower back appears more sensitive to cold, wetness and thermal displeasure). Second, these sensory patterns seem independent from the regional variations in skin cooling (i.e. regions which show greater skin cooling, such as the lateral chest, are not necessarily the ones in which the stimulus was perceived as colder and more often wet). [From Filingeri et al. (99); (c) The American Physiological Society].

Figure 4. A cold thermoreceptors in the glabrous skin of the cat's nose. Panel A shows a vertical semithin section (650x) through a marked area in the cat's nose, where a cold thermoreceptors is identified (black arrows). A lamellate encapsulated receptor with a small capsule space (Ic) is located in the marked field too, but lies about 80 microns deeper in the skin near the epidermal columnar ridge (cr). Capillary (cp). Panel B shows the same cold receptor (electron micrograph; 20000x) with its axon (tax) entering into the basal layer of the epidermis. The axoplasm contains numerous mitochondria (mi) and some scattered glycogen particles. The basal lamina (bl) of the epidermis fuses with the basal lamina of the receptor axon (arrows). Schwann cell (sc), collagen fibers of the papillary connective tissue (cf), tonofibrils (tf) of the epithelial cells are also marked. Panel $\mathrm{C}$ shows a schematic representation of the marked cold receptor in panel $\mathrm{A}$ and various mechanoreceptors in the glabrous skin of the cat's nose. The cold receptor (cld) is located in the top of a dermal papilla and its terminals penetrate into the basal layer of the epidermis. Merkel cell neurite complexes $(\mathrm{m})$ are sometimes located in the epithelium of the epidermal columnar ridge (cr). Lamellate encapsulated receptors with a capsule space (Ic) are present in the connective tissue below. Tip of the wire hole (w), used to slice the sample. Adhesive ridge (at). Panel D shows a schematic representation of the cold receptor axon shown in panel $B$. The terminal protrudes into a basal epidermal cell. The Schwann cell cover and basal lamina fuse with the epidermis. The typical structures of the receptor matrix are present below the receptor membrane. Receptor axon (tax), Schwann cell (sc), epidermis (e), papillary connective tissue (pct), basal lamina (bl) [From Hensel (145); with kind permission from Springer Science and Business Media].

Figure 5. Responses of human cold sensitive (A, B and E) unmyelinated C-fibers and (C) myelinated $A \bar{\delta}$-fibers to $a(D)$ standard cooling staircase. Panel $A$ and $B$ show two $C$-fibers discharging upon cooling all the way down to $0^{\circ} \mathrm{C}$. Panel $\mathrm{C}$ shows one $A \delta$-fiber discharging upon cooling but stopping firing at about $14^{\circ} \mathrm{C}$ [Reprinted from Campero et al. (39) with permission 
from John Wiley \& Sons, Inc.]. Panel E shows responses of a single cold sensitive C-fiber to $10 \mathrm{~s}$ temperature pulses. Top trace: full response to a temperature pulse from a baseline skin temperature of $35^{\circ} \mathrm{C}$ to $30^{\circ} \mathrm{C}$, with the temperature profile shown below. Underneath traces: expanded 1-s sections of responses showing peak phasic (left) and tonic (right) discharges, for temperature steps (rate of change: $10^{\circ} \mathrm{C} \mathrm{s}^{-1}$ ) from $35^{\circ} \mathrm{C}$ to the temperatures indicated [Reprinted from Campero et al. (40) with permission from John Wiley \& Sons, Inc.].

Figure 6. Paradoxical discharge of human and primate cold sensitive fibers in response to heat stimuli. Panel A shows activity recorded in a human cold sensitive $\mathrm{C}$-fiber in response to skin warming (10-s pulse) from 35 to $40^{\circ} \mathrm{C}$. Panel B shows activity in the same fiber in response to skin warming from 35 to $45^{\circ} \mathrm{C}$. It can be observed that, while innocuous warming ( 35 to $40^{\circ} \mathrm{C}$ ) does not activate the unit, skin warming beyond the noxious threshold $\left(<42^{\circ} \mathrm{C}\right)$ results in a paradoxical discharge in this cold sensitive unit. Panel $\mathrm{C}$ shows a 1-s expansion of the period marked by the open bar in panel $\mathrm{B}$, highlighting the bursting pattern of response for this human C-fiber [Reprinted from Campero et al. (40) with permission from John Wiley \& Sons, Inc.]. Panel $D$ shows paradoxical discharge in primate cold sensitive A-fibers in response to heat stimuli (i.e. $>47^{\circ} \mathrm{C}$ ) and their modulation by different core temperatures. The graph shows the proportion of sampled cold fibers which responded to stimuli of different intensities. It can be observed that the skin warming threshold for this paradoxical discharge decreases (from 49 to $41^{\circ} \mathrm{C}$ ) as core temperature increases (from 37 to $39^{\circ} \mathrm{C}$ ) [From Long (201); (C) The American Physiological Society].

Figure 7. Static thermal sensitivity of primate and human warm sensitive C-fibers. Panel A shows average static impulse frequency of two populations of single warm fibers from hairy skin of foot in rhesus monkey as function of constant temperatures. The dashed line indicates shift from regular go irregular discharge or bursts. It can be observed that in one population, the function of discharge rate versus steady state stimulus temperature follows a bell-shaped curve, while in the other population, steady frequency increases continuously with rising skin temperature up to 44 ${ }^{\circ} \mathrm{C}$ [From Hensel and Iggo (147); with kind permission from Springer Science and Business Media]. Panel B shows total number of spikes for a human warm sensitive C-fiber during the first $3 \mathrm{~s}$ after the application of thermal stimuli (filled circles) in comparison with the averaged total impulses during corresponding periods for 15 polymodal nociceptive C-fibers (triangles) [From Hallin et al. (134); adapted by permission from BMJ Publishing Group Limited].

Figure 8. Impulse frequency as a function of time in a single cold sensitive A-fiber isolated from human radial nerve during skin cooling and rewarming [From Hensel and Boman (146); (c The American Physiological Society].

Figure 9. Dynamic thermal sensitivity of primate cold sensitive A-fibers and warm sensitive Cfibers. Panel A shows peristimulus time histograms for a cold sensitive A-fiber computed using spike trains evoked by skin cooling. The cool-stimulus intensity-rate series consisted of decreases in temperature of $0.5,1,2$ and $5^{\circ} \mathrm{C}$ from an adapting temperature of $30^{\circ} \mathrm{C}$ and at rates of (i) 2 and (ii) $0.4^{\circ} \mathrm{C} \cdot \mathrm{s}^{-1}$ [From Kenshalo and Duclaux (174); (c) The American Physiological Society]. Panel B shows peristimulus time histograms for a warm sensitive C-fibers computed using spike trains evoked by skin warming. (i) Responses to warming pulses of intensities 2, 4, 6 and $8^{\circ} \mathrm{C}$ from a baseline skin temperature of $34^{\circ} \mathrm{C}$. The profile of the warming pulses is shown in black at the bottom of the graph. It can be observed that, although the fibers' responses increased in an orderly fashion with increasing stimulus intensity, the temporal profile of the response was remarkably constant during the first $4 \mathrm{~s}$ of stimulation. This can be further observed in (ii) and (iii), in which the fiber's responses to stimuli of intensities of (ii) 4 and $2^{\circ} \mathrm{C}$ and of (iii) 4 and $8^{\circ} \mathrm{C}$, have been normalized by equating the 4-s cumulative impulse counts of the two responses. Such normalization show how the temporal profile of the responses to warming pulses of different intensities match well during the first $4 \mathrm{~s}$ of stimulation. As that, indicating that the rate of frequency increase in these fibers appears to be the same regardless of the intensity of the temperature change. Such observation implies that this parameter is not the main one used by the brain to determine the magnitude of temperature change [From Darian-Smith et al. (74); ( ) The American Physiological Society]. 
Figure 10. Cold sensitive lamina I neurons in the cat's spinal cord. Panel A shows a photomicrograph of a lesion marking the recording site (black arrow) of a thermosensitive lamina I spinothalamic neuron in the lumbar-7dorsal horn of the cat' spinal cord. Bar $=0.5 \mathrm{~mm}$. Panel B shows two histogram records ( $\mathrm{i}$ and ii) highlighting the reproducibility of the response of this coldsensitive lamina I thermosensitive neuron to the standard cooling/warming stimulus sequence (shown by the traces at the bottom of each record indicating skin-thermode temperature). (iii) Summary of the mean discharge rates at each temperature for 4 applications of the same stimulus [From Craig et al. (65); @ The American Physiological Society]. Panel C shows camera lucida reconstructions of the full dendritic extent of 3 different categories of lamina I neurons identified in the cat' spinal cord: 1) fusiform nociceptive (NS) specific; 2) pyramidal (COLD) thermo-receptive specific; 3) multipolar heat-pinch-cold (HPC) specific (note: rostral is left, medial is up) [Reprinted by permission from Macmillan Publishers Ltd: Nature Neuroscience (Han et al., copyright 1998) (135)].

Figure 11. Cold-sensitive Lamina I spino-thalamic neuron in the monkey's spinal cord. Panel A shows an antidromic response in this spino-thalamic neuron to stimulation pulses applied from an electrode in the ventral medial nucleus of the thalamus. Panel B shows 2 periods of inhibition of the spontaneous firing of this neuron upon radiant warming applied on the unit's receptive field (i.e. glabrous skin of the foot), which is illustrated in panel C (black region indicates maximal sensitivity). Panel $D$ shows dynamic responses of this spinal cold sensitive neuron to progressive cooling steps of $4^{\circ} \mathrm{C}$, from 34.5 (baseline) to $12.5^{\circ} \mathrm{C}$. Responses to warming pulses are shown in the second half of the record, where immediate reductions in tonic firing upon warming are observable, as well as a characteristic paradoxical discharge upon noxious heating $\left(>47^{\circ} \mathrm{C}\right)$. Panel E shows relationship between dynamic and static responses of this neuron and skinthermode interface temperature. For warming pulses the values plotted represent the mean firma rate during the total duration of the heat pulse. Note that response at $40^{\circ} \mathrm{C}$ is inhibition (less-than baseline firing rate at 34\%) [From Dostrovsky and Craig (88); ( ) The American Physiological Society].

Figure 12. Warm sensitive lamina I neurons in the cat's spinal cord. Panel A shows responses of a single warm sensitive neuron to innocuous $\left(38.7^{\circ} \mathrm{C}\right)$ and noxious $\left(53.0^{\circ} \mathrm{C}\right)$ skin warming respectively. Specifically, firing rate (1-s bins), temperature profile and single unit recording are shown. Panel B shows individual temperature stimulus-response curves for 8 different group warm sensitive lamina I neurons and panel $C$ shows group mean $( \pm 1$ standard deviation) for the same group $(n=8)$ [Reprinted from Andrew and Craig (5) with permission from John Wiley \& Sons, Inc.].

Figure 13. Micro-stimulation of the posterior and inferior core of the ventral caudal nucleus (a portion of the ventral posterior lateral nucleus) of the human hypothalamus evokes non-painful thermal sensations in humans. Eleven patients undergoing stereotactic surgery for the treatment of movement disorders and pain were trained to describe sensations evoked intraoperatively by thalamic microstimulation. The figure shows maps of receptive and projected fields for trajectories in the region of the ventral caudal nucleus in a single patient. The stereotactic coordinates of the anterior commissure (AC) and posterior commissure (PC) were determined by computer-assisted tomography. These coordinates were used to generate maps of the human thalamus in sagittal section that had been transformed to match the AC-PC line in that patient. Panels $A$ shows positions of the trajectories relative to nuclear boundaries as predicted radiologically from the position of the anterior commissure-posterior commissure (AC-PC) line. The AC-PC line is indicated by the horizontal line in the panel; the trajectories are shown by the 2 oblique lines. The positions of nuclei are inferred from the AC-PC line and therefore are only an approximate indicator of nuclear location (anatomic names; Vc: ventralis caudalis; Vcpor: ventralis caudalis portae; Vcpc: ventralis caudalis parvocellularis; Lim: limitans; MG: medial geniculate; ML: medial lemniscus; WM: white matter below the ventral nuclear group). Panel B shows location of single neurons, stimulation sites and trajectories (S 1 and S2) relative to the PC. The locations of stimulation sites are indicated by ticks to the left of the trajectory; the locations of single neurons are indicated by ticks to the right of the trajectory. Single neurons with receptive fields (RFs) are indicated by long ticks; those without are indicated by short ticks. The 
core thalamic region is defined as the area where the majority of ticks to the right of the trajectory are long. The quality of the evoked sensation is indicated by the symbol at the end of the tick to the left of the trajectory. Square: paresthetic sensation was evoked at that site. Open circle: sensation of non-painful heat. No symbol: no sensation was evoked. Scales are as indicated. Each site where a neuron was recorded or stimulation was carried out or both is indicated by the same number in panels B and C. In panel C, S1 and S2 show the site number, projected field (PF), and RF for that site. The threshold (in microamperes) is indicated below the PF diagram [From Lenz and Seike (195); (c) The American Physiological Society].

Figure 14. Positron emission topography imaging performed during tonic cooling of the hand (range of 33 to $22{ }^{\circ} \mathrm{C}$ ) in humans and activation of the insular cortex. Panel A shows the relationship between: (top graph) magnitude estimate of the perceived intensity of cold (following each scan using an open-ended scale of zero to ten) and stimuli temperatures; (bottom graph) average regional cerebral blood flow activity in left (contralateral) insular cortex and stimuli temperatures. Panel C shows the location of "thermosensory cortex" in the dorsal margin of left (contralateral) insular cortex (identified by regression analysis of regional cerebral blood flow activation with stimulus temperature) in frontal, axial and sagittal views (note that in the axial view, this site seems parietal simply because the insula cannot be seen). The activation in right anterior insula is also visible in the axial view. It can be observed that cortical activity in such region strongly correlates with human discrimination of the intensity of the thermal stimulus [Reprinted by permission from Macmillan Publishers Ltd: Nature Neuroscience (Craig et al., copyright 2000) (62)].

Figure 15. Evoked potentials recorded directly from the brain in response to an innocuous cold stimulus applied to the contralateral hand of two female patients who had subdural grids implanted for surgical treatment of medically intractable complex partial seizures. Panel A shows: (top) temperature profile of the cold stimulus used; (bottom) the maximal potential recorded over the parietal operculum. Panel B shows the location of the recording electrodes. Cold-evoked potentials were recorded from 3 rows of 6 electrodes labeled C (1-6), D (1-6) and E (1-6) (CS: central sulcus; IFS: inferior frontal gyrus; SFS: superior frontal sulcus; PreCS: precentral sulcus; PostCS post central sulcus; SF: sylvian fissure). Panels C, D and E shows cold-evoked potentials recorded from each electrode in each row. Overlays of potentials recorded from 2 adjacent electrodes with large cold-evoked potentials for each row are shown as the bottom tracing in the corresponding C, D and E panels. It can be observed that the largest cold-evoked potentials were recoded from electrodes adjacent to the sylvian fissure [From Greenspan et al. (129); @ The American Physiological Society].

Figure 16. Tridimensional structure of the TRPV1 ion channel (determined by electron cryomicroscopy). Panel A present a linear diagram depicting major structural domains, color coded to match ribbon diagrams in panel B. The TRPV1 channel contains six transmembrane spanning regions (S) with a pore-forming reentrant loop between the fifth (S5) and the sixth (S6). Both the carboxyl (C-) and amino (containing 3 to 5 ankyrin repeats) termini are intracellular (dashed boxes denote regions for which density was not observed, e.g. first two ankyrin repeats, or where specific residues could not be definitively assigned, e.g. C-terminal b-strand). Panel B presents diagrams showing three different tridimensional views of a TRPV1 channel [Reprinted by permission from Macmillan Publishers Ltd: Nature (Liao et al., copyright 2013) (197)].

Figure 17. The capsaicin receptor vanilloid receptor 1, VR1 (subsequently renamed TRPV1) activated by noxious thermal stimuli. Panel A shows Xenopus (i.e. clawed frog) oocytes cells transiently transfected with TRPV1 exhibit a pronounced increase in cytoplasmic free calcium when transiently exposed to a peak temperature of $45^{\circ} \mathrm{C}$. The same cells did not respond to heat when transfected with vector alone (pcDNA3). Relative calcium concentrations are indicated by the color bar. Panel B shows results from whole-cell patch-clamp analysis of TRPV1-transfected Xenopus oocytes cells. It can be observed that inward currents are generated in response to both heat and capsaicin. The temperature of the bath medium was raised from 22 to $48^{\circ} \mathrm{C}$ (heat), and then restored to $22^{\circ} \mathrm{C}$, after which capsaicin $(0.5 \mathrm{mM})$ was added to the bath. Stimulus-induced current-voltage relations are shown on the right. Panel C shows that TRPV1 expressed in Xenopus oocytes is activated by noxious but not innocuous warm temperatures. Two-electrode 
voltage-clamp was performed in oocytes injected with either TRPV1 cRNA or water while the perfusate temperature was raised from 22 to $-45^{\circ} \mathrm{C}$. The asterisk indicates a significant difference from water-injected oocytes. Panel D shows the inhibitory effect of Ruthenium red (RR) (a potent inhibitor of intracellular calcium release) on heat- and capsaicin-evoked responses in TRPV1expressing oocytes [Reprinted by permission from Macmillan Publishers Ltd: Nature (Caterina et al., copyright 1997) (44)].

Figure 18. TRPM8 ion channel as the principal detector of environmental cold. Results are reported from analysis of cold-evoked responses in cultured sensory neurons and intact sensory nerve fibers from TRPM8-deficient mice. Also, behavioral discriminatory ability between cold and warm surfaces, and responses to evaporative cooling, were analyzed in TRPM8 mutant mice. Panel A shows immunostaining of trigeminal ganglia (left), corneal afferents (middle), and spinal cord dorsal horn (right) with anti-TRPM8 (green) and anti-TRPV1 (red) antibodies in TRPM8deficient mice. It can be observed that these animals present a selective loss of TRPM8 expression (scale bars, $50 \mathrm{~mm}$ ). Panel B shows: (i) responses of trigeminal neurons to menthol (TRPM8 agonist), capsaicin (TRPV1 agonist) and Potassium Cloride (KCl) in TRPM8 expressing $(+/+)$ and lacking (-/-) mice (note: no menthol-sensitivity is observable in TRPM8-deficient neurons); (ii) prevalence of sensory neurons responding to capsaicin, mustard oil, menthol and icillin in TRPM8 expressing (+/+) and lacking (-/-) mice (note: significantly reduced proportion of neurons responding to menthol and icillin in TRPM8-deficient neurons); (iii) responses of trigeminal neurons to cold and menthol (green bar) in TRPM8 expressing (+/+) and lacking (-/-) mice (note: no cold and menthol-sensitivity is observable in TRPM8-deficient neurons; dotted line: menthol-insensitive neurons); (iv) comparison of cold and heat sensitivity in wild type, TRPM8- and TRPV1-deficients mice (note: cold and heat sensitivities are selectively reduced in TRPM8- and TRPV1-deficient mice respectively). Panel C shows: (i) cooling-induced responses in cutaneous C-fibers; (ii) percentage of cold-activated C-fibers; (iii) cooling-induced responses in cutaneous A-mechanoreceptive fibers; (iv) percentage of cold-activated fibers Amechanoreceptive fibers, in wild type and TRPM8-deficient mice [Reprinted by permission from Macmillan Publishers Ltd: Nature (Bautista et al., copyright 2007) (11)].

Figure 19. A schematic summary of the molecular, neuroanatomical and neurophysiological bases of skin thermal sensations in humans. Human hairy skin comprises of a number of first order neurons innervating the epidermal layer of the skin. Innocuous warmth is encoded by unmyelinated C-fibers expressing TRPV1 ion channels. Expression of warm sensitive potassium channels (TREK/TRAAK) likely contribute to membrane resting potentials. The presence of warmth sensitive TRPV3 and TRPV4 ion channels expressed in skin keratinocytes, contributing to warmth transduction via ATP-mediated signaling mechanisms to C-fibers could also contribute to warmth detection. Innocuous cold is encoded primarily by myelinated $A \delta$-fibers expressing TRPM8 ion channels and cold sensitive sodium channels (Nav1.8; contributing to membrane resting potentials). Myelinated A $\beta$-fibers (mechanoreceptors) expressing TRPM8 ion channels show uncorrelated responses to cold temperatures. Unmyelinated cold and heat sensitive C-2 fibers, expressing cold sensitive TRPM8, heat sensitive TRPV1 and potentially cold sensitive TRPM3 and TRPA1 ion channels, encode noxious cold $\left(<15^{\circ} \mathrm{C}\right)$ and heat $\left(>45^{\circ} \mathrm{C}\right)$. In panel $\mathrm{A}$ and $B$, temperature-dependent changes in discharge frequency of first order thermosensitive neurons and in TRP-mediated ionic conductance, are shown. Neuroanatomically, the specific ascending thermosensory pathway which allows humans to peripherally encode and centrally process skin thermal sensations comprises of: 1 ) first order thermo-sensitive $A \delta-, C$ - and C2-fibers, terminating in the spinal cord Lamina I, and synapsing with cold- and warm-sensitive and heat-pinch-cold sensitive second order neurons; 2) second order lamina I spino-thalamic neurons, ascending contralaterally along the anterolateral columns of the spinothalamic tract, and terminating in the posterior part of the ventral medial nucleus of the thalamus (VMpo); 3) third order thermosensitive neurons, located in posterior part of the ventral medial nucleus of the thalamus (VMpo) and projecting to the posterior insular cortex, i.e. the main thermosensory cortex. Functionally, this neurophysiological pathway sub serves peripheral and central mechanisms underpinning our ability to characterize both the discriminative and affective components of skin thermal sensations in the context of thermal behavior. Potential interactions with other cortical (e.g. somatosensory cortex) and sub cortical regions (e.g. nucleus of the tractus solitarious, hypothalamus), receiving 
sensory inputs from both thermoreceptive and mechanoreceptive fibers (note: these ascend to the somatosensory cortex via the medial lemniscus and the thalamic ventroposterior nuclei), likely contribute to modulate and enrich thermal processing in the context of multimodal (e.g. thermaltactile) somatosensory interactions and human thermo-sensory experience.

Figure 20. Specific ranges and rates of local skin cooling drive the perception of local skin wetness. This figure presents psychophysical results related to the application of 6 progressively more intense cold-dry stimuli (via a thermode) on the forearm of blindfolded females, who reported their stimulus-driven wetness perception upon contact cooling. Panel A shows relative drops in skin temperature from baseline and corresponding cooling rates as a result of each of the six cold-dry stimuli. Panel B shows wetness perception scores recorded as a result of each of the six cold-dry stimuli (phase B) and after removal of the stimulus (i.e. bare skin phase, C). Skin cooling rates corresponding to each stimulus are reported between brackets. The point " 1 " of the wetness perception scale corresponds to the threshold set to identify perceived skin wetness. It can be observed that cold-dry stimuli producing skin cooling rates in the range of 0.14 to $0.41^{\circ} \mathrm{C} s$ ${ }^{1}$ induced a clear illusion of skin wetness [Reprinted from Filingeri et al. (102) with permission from Elsevier].

Figure 21. Neurophysiological model of cutaneous wetness sensitivity. Mechano sensitive Aß-, cold sensitive $A \delta$ - and warm sensitive $C$-fibers and their projections from the skin, through peripheral nerve, spinal cord (via the dorsal-column medial lemniscal pathway and the spinothalamic tract), thalamus and cerebral cortex (including the primary and secondary somatosensory cortices SI and SII, the insular cortex and the posterior parietal lobe) are shown. Panel $A$ and $B$ shows the neural model of wetness sensitivity (consisting of $A \delta$ and $A ß$ afferents) under normal and under selective reduction in the activity of A-nerve fibers respectively. Panel $C$, $E$ and $G$ show the pathways for wetness sensitivity during static contact with warm, neutral and cold moisture. Panel $\mathrm{D}, \mathrm{F}$ and $\mathrm{H}$ shows the pathways for wetness sensitivity during dynamic contact with moisture [From Filingeri et al. (98); (c) The American Physiological Society].

Figure 22. Ocular surface wetness as regulated by TRPM8-dependent cold thermoreceptors in the cornea. This figure presents responses of mice corneal neurons to cooling ramps and menthol, as well as dependence of tear secretion rate on corneal temperature, as recorded in wild type mice expressing TRPM8 ion channels $\left(\mathrm{TRPMB}^{+/+}\right)$, in mutant mice presenting a reduced expression of TRPM8 ion channels (TRPM8 ${ }^{+-}$), and in TRPM8-deficient mice. Panel A shows mean firing frequency as a result of a cooling ramp (left) and menthol application (right). It can be observed that $\mathrm{TRPM}^{+/-}$and $\mathrm{TRPM}^{-{ }^{--}}$corneal neurons present reduced and absent response to cooling and menthol respectively. Panel $B$ shows mean firing frequency as a result of the same cooling ramp in corneal neurons from mice expressing $\left(\mathrm{TRPA}^{+/+}\right)$or deficit of $\left(\mathrm{TRPA}^{-1}\right)$ the noxious-cold sensitive TRPA1 ion channel. It can be observed that no changes in innocuous cold sensitive of corneal neurons occur as a result of the absence of TRPA1, indicating a lack of involvement of this channel in the transduction of innocuous corneal cooling. Panel C shows basal tearing rate (mean wetted length of the phenol red thread in $\mathrm{mm}$ ) measured in the eyes of anesthetized mice exposed to environmental temperatures of 25 and $42^{\circ} \mathrm{C}$ that modified their corneal surface temperature to the values indicated. It can be observed that increases in tearing rate during corneal cooling occurring in wild type (WT) mice (black bar), is abolished in TRPM8 lacking mice (TRPM8 ${ }^{-1}$; red bar), while conserved in TRPA1 lacking mice (TRPA1 ${ }^{-1}$; blue bar). Panel $D$ shows changes in tearing rate as a result of capsaicin $(1 \mu \mathrm{M})$ and AITC $(500 \mu \mathrm{M})$ (two well-known chemo irritants) application to the cornea of wild type (WT) mice (black bar) and TRPM8 lacking mice (TRPM8 ${ }^{-1}$; red bar). It can be observed that in both TRPM8 expressing and lacking mice, irritation-induced tearing is unaltered, indicating that while TRPM8 likely controls basal tearing, this is not involved in reflex responses to noxious agents. Panel D shows tearing rates at different corneal temperatures in 11 healthy humans. It can be observed that, as corneal temperature increases, tearing rate decreases [Reprinted by permission from Macmillan Publishers Ltd: Nature Medicine (Parra et al., copyright 2010) (240)].

Figure 23. Conceptual model of human hygrosensation. The model comprises biophysical (i.e. thermal and tactile inputs induced by the presence of moisture on the skin), neurophysiological 
(i.e. central integration of afferents inputs from thermo-sensitive TRP ion channels and nerve fibers and mechano-sensitive Degenerin/Epithelial sodium channels (DEG/ENaC) ion channels and nerve fibers) and psychophysiological mechanisms (i.e. perceptual inference operated by cortical and sub-cortical somatosensory and association areas) which allow humidity and wetness detection in humans. The skin's contact with moisture generates thermal and tactile inputs which are peripherally integrated by specific nervous structures. These inputs evoke thermal and tactile sensations which, in the absence of specific hygroreceptors, are associated to the perception of skin wetness. Repeated exposures to these stimuli (i.e. sensory experience) contribute to generate a neural representation of a typical wet stimulus via learning mechanisms. At this point, only if the learnt combination of stimuli (i.e. coldness and stickiness), as coded by the specific neural afferents (i.e. A-nerve fibers) is presented, wetness will be sensed. In the occurrence of physical wetness on the skin, the bottom-up processes (i.e. combination of thermal and mechanical sensory afferents) as well as the top-down ones (i.e. inference of the potential perception based on the neural representation of a typical wet stimulus) might therefore interact in giving rise (or not) to the perception of wetness [From Filingeri et al. (101)].

\section{Further Reading}

Click here to insert Further reading text

\section{Cross-References}

Coping with thermal challenges: physiological adaptations to environmental temperatures

Neural control of thermoregulation

Sense of touch: performance and peripheral neural processes (legacy)

Transient receptor potential (TRP) channels and thermoregulation

Thermometry, calorimetry, and mean body temperature during heat stress

\section{Supplementary Information}

Click here to insert Supplementary information text if applicable

Before saving and uploading your text to ScholarOne Manuscripts at

http://mc.manuscriptcentral.com/cphy,

please turn off the comments balloons by clicking on "Delete all Comments"

in the Comments section of the Review tab of Microsoft Word. 